\title{
Tennis elbow
}

Citation for published version (APA):

Verhaar, J. A. N. (1992). Tennis elbow. [Doctoral Thesis, Maastricht University]. Datawyse / Universitaire Pers Maastricht. https://doi.org/10.26481/dis.19920904jv

\section{Document status and date:}

Published: 01/01/1992

DOI:

10.26481/dis.19920904jv

Document Version:

Publisher's PDF, also known as Version of record

\section{Please check the document version of this publication:}

- A submitted manuscript is the version of the article upon submission and before peer-review. There can be important differences between the submitted version and the official published version of record.

People interested in the research are advised to contact the author for the final version of the publication, or visit the DOI to the publisher's website.

- The final author version and the galley proof are versions of the publication after peer review.

- The final published version features the final layout of the paper including the volume, issue and page numbers.

Link to publication

\footnotetext{
General rights rights.

- You may freely distribute the URL identifying the publication in the public portal. please follow below link for the End User Agreement:

www.umlib.nl/taverne-license

Take down policy

If you believe that this document breaches copyright please contact us at:

repository@maastrichtuniversity.nl

providing details and we will investigate your claim.
}

Copyright and moral rights for the publications made accessible in the public portal are retained by the authors and/or other copyright owners and it is a condition of accessing publications that users recognise and abide by the legal requirements associated with these

- Users may download and print one copy of any publication from the public portal for the purpose of private study or research.

- You may not further distribute the material or use it for any profit-making activity or commercial gain

If the publication is distributed under the terms of Article $25 \mathrm{fa}$ of the Dutch Copyright Act, indicated by the "Taverne" license above, 


\section{TENNIS ELBOW}


(c) 1992 J.A.N. Verhaar

No part of this book may be reproduced or in any way commercially used without written permission of the author.

CIP.DATA KONINKLUJKE BHBLOTHEEK, DEN HAAG

Verthat, Johannes Albertus Nicolaas

Teninis elbow/Johannes Albertus Nicoladis Verharar. -

Matstricht: Universitaire Pers Maastricht. - III

Thesess Madstricht. - With ref. - With summary in Dutch.

ISBN $90-5278-034-X$

NUGH 742

Subject headings: temis elbow/ radial tumel syndrome.

Boekverzorging: Datawyse / Universitaire Pers Maastricht Omslagontwerp: Jan Eggen Illustraties: Hans Rensema, RL-anatomie, Maastricht Correctie engels: drs. Jan Klerx, Maastricht Druk: Krips Repro Meppel

This publication was financially supported by grants from "het Nationaal Reumafonds", the Dutch Orthopaedic Association, Stryker Europe BV, 3M, Ortomed, Zimmer, Schering, Sanofi, Smith and Nephew and Somas. 


\section{TENNIS ELBOW}

\section{PROEFSCHRIFT}

ter verkrijging van de graad van doctor aan de Rijksuniversiteit Limburg te Maastricht, op gezag van de Rector Magnificus, Prof.Mr. M.J. Cohen, volgens het besluit van het College van Deksnen, in het openbaar te verdedigen op vrijdag, 4 september 1992 om 16.00 uur

door

JOHANNES ALBERTUS NICOLAAS VERHAAR

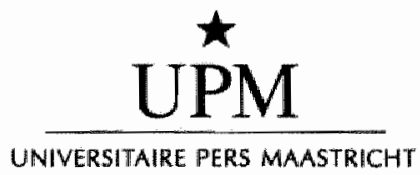


Promotor:

Prof.dr. A.J. van der Linden

Co-promotores:

Dir. H. van Mameren

Dr. G.H.L.M. Walenkamp

Beoordelingscommissie:

Prof.dr. J.W. van Ree (Voorzitter)

Prof.dr. J. Drukker

Prof.dr. G. Kootstra

Dr. A.J.G. Nollen (St. Joseph ziekenhuis Veldhoven)

Prof.dr. P.M. Rozing (Rijksuniversiteit Leiden) 


\section{Contents}

INTRODUCTION

\section{Chapter 1}

\section{SURVEY OF THE LITERATURE}

1.1. Etiology and pathology ............................................................ 14

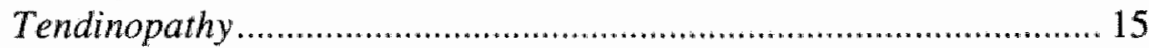

Intra-articular lesions ................................................................... 17

Compression of the radial nerve ................................................... 18

Cervical radiculopathy .............................................................. 18

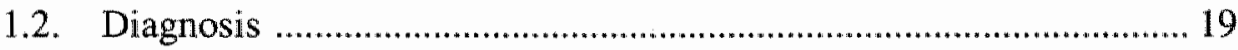

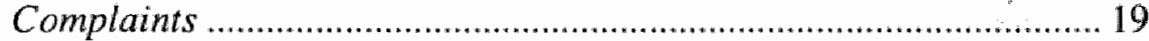

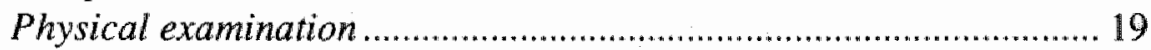

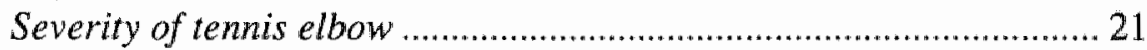

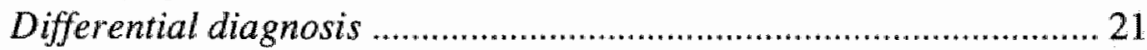

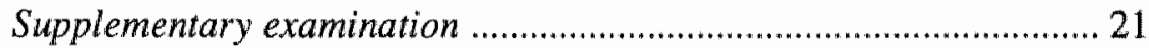

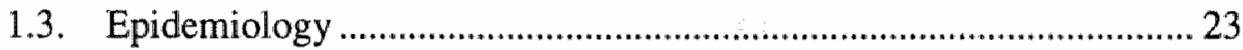

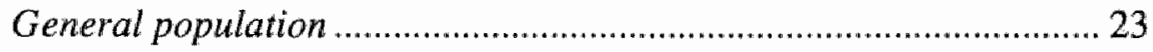

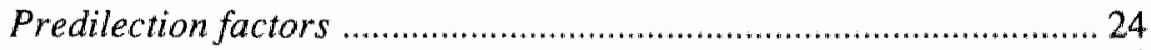

Economic aspects ................................................................... 25

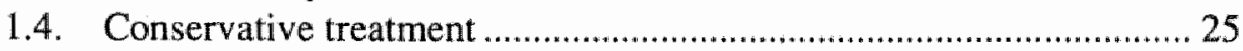

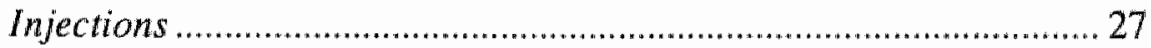

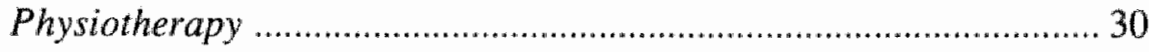

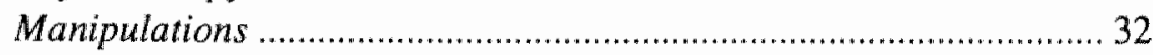

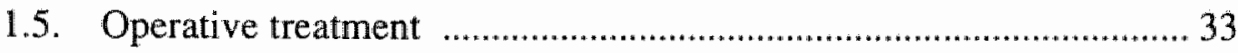

Operative techniques .................................................................. 33

Results of operative treatment ................................................... 36

Complications ............................................................................. 37

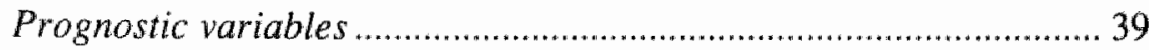

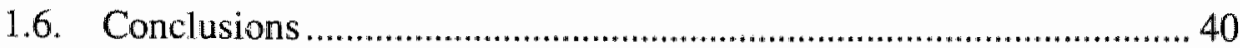




\section{Chapter 2}

\section{ANATOMICAL ASPECTS OF TENNIS ELBOW}

2.1. Introduction....

2.2. Anatomy of the lateral elbow region ............................................. 42

2.3. Action of the posterior antebrachial muscle group .......................... 47

2.4. Lateral Cubital Force Transmission System .................................... 48

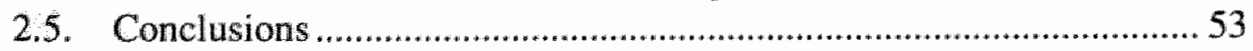

\section{Chapter 3}

\section{EPIDEMIOLOGY OF TENNIS ELBOW}

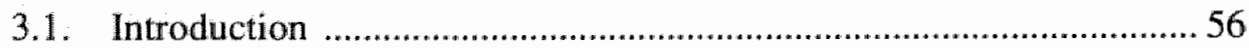

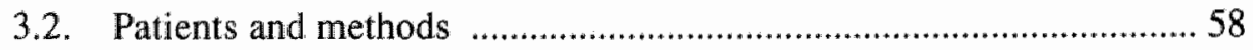

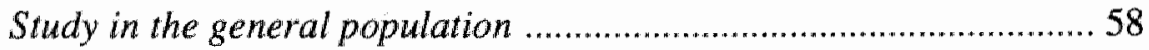

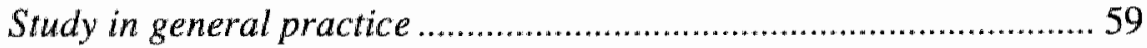

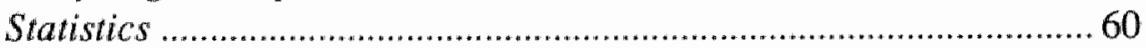

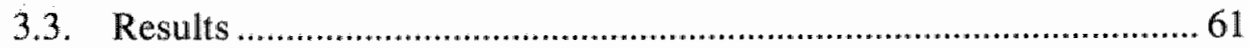

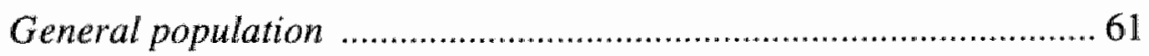

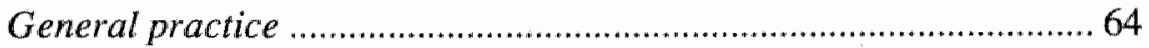

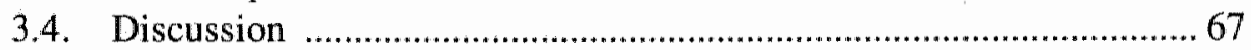

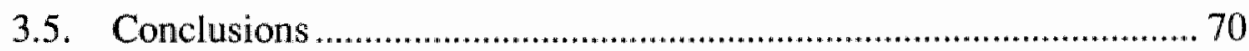

\section{Chapter 4}

CONSERVATIVE TREATMENT OF TENNIS ELBOW

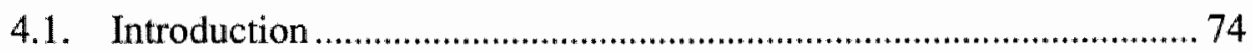

4.2. Patients and methods ....................................................................... 75

Selection of patients and randomization ...................................... 75

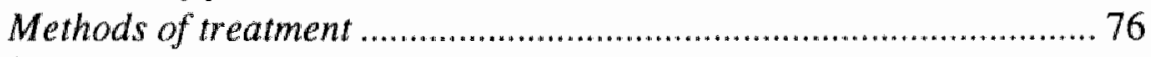

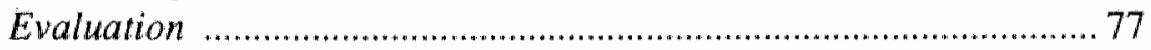

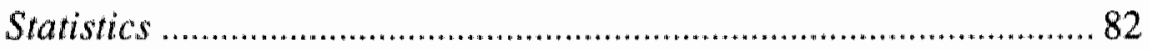

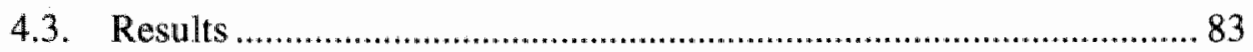

Pretreatment variables ................................................................ 83

Evaluation of treatment at 6 weeks ................................................ 87

Additional treatments between 6 and 52 weeks ............................ 96

Evaluation of treatment at 52 weeks ............................................. 98

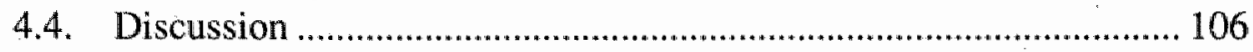

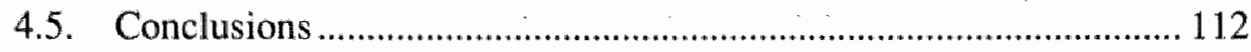




\section{Chapter 5}

\section{OPERATIVE TREATMENT OF TENNIS ELBOW}

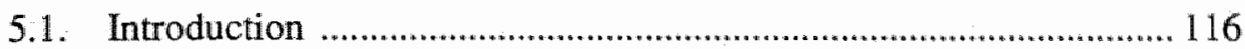

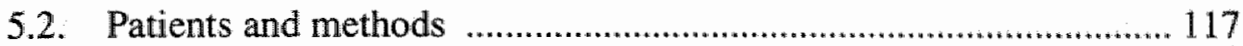

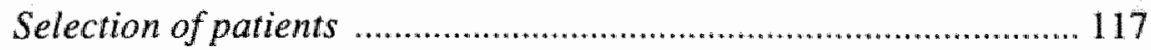

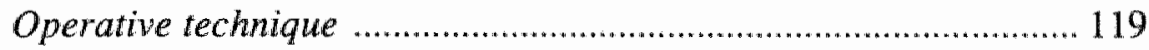

Evaluation of the results of treatment .......................................... 120

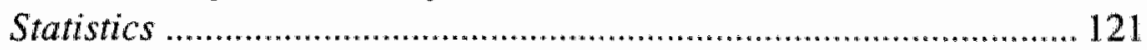

Histology of extensor origin ....................................................... 121

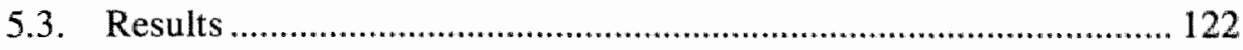

Findings at operation ............................................................ 122

Histology of extensor origin .......................................................... 122

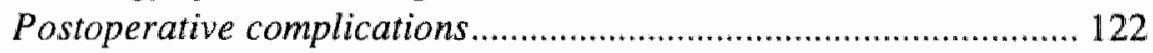

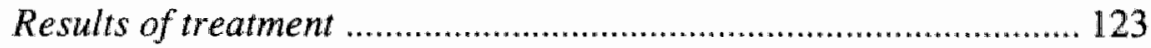

Prognostic factors ................................................................. 129

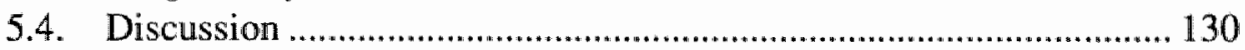

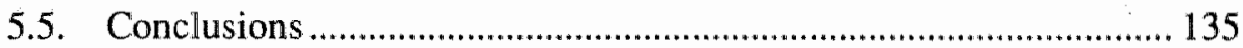

\section{Chapter 6}

\section{THE LATERAL CUBITAL FORCE TRANSMISSION SYSTEM}

6.1. Introduction .......................................................................... 138

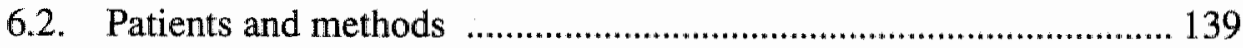

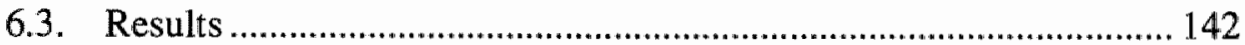

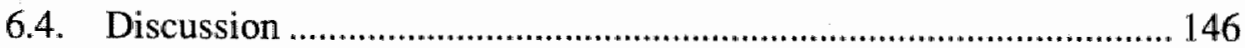

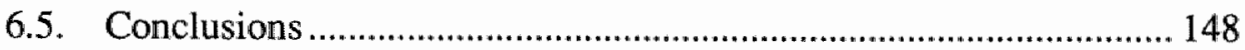

\section{Chapter 7}

\section{RADIAL TUNNEL SYNDROME}

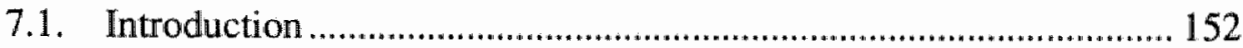

7.2. Patients and methods ................................................................ 153

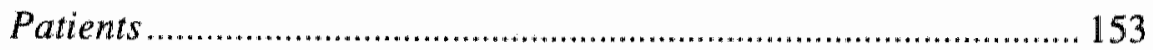

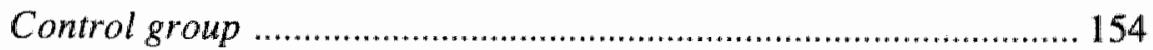

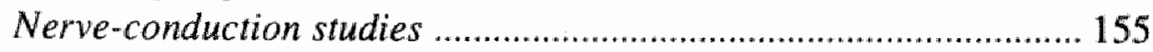

Concentric-needle electromyography .......................................... 156

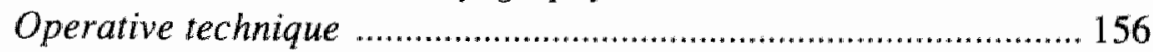

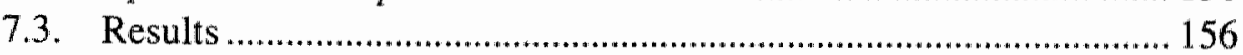




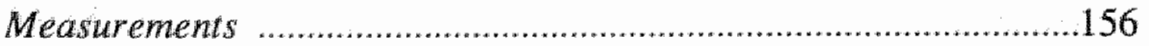

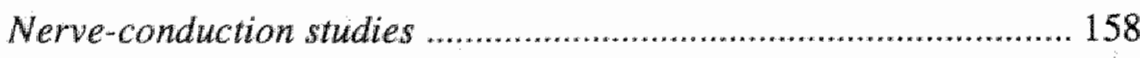

Concentric-needle electromyography ........................................ 159

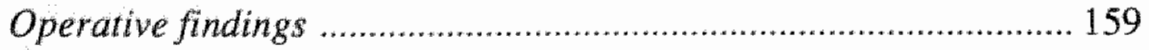

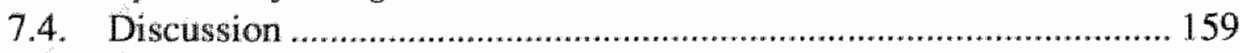

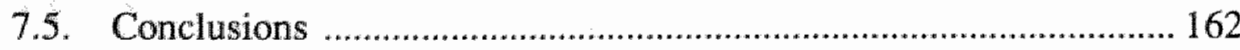

\section{Chapter 8}

GENERAL CONCLUSIONS

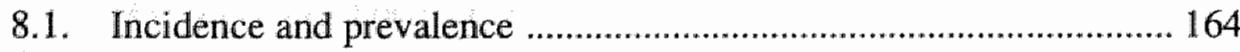

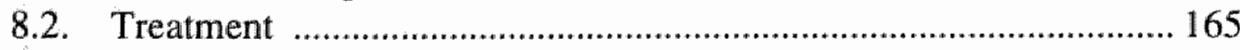

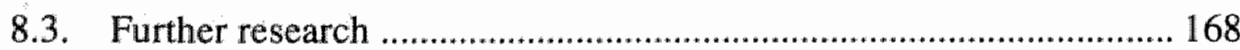

\section{Chapter 9}

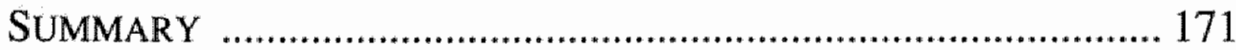

\section{Chapter 10}

SAMENVATTING

\section{Chapter 11}

REFERENCES

TENSLOTTE 
Tennis elbow is a common, painful, often intractable and imperfectly understood condition, affecting the attachments of the extensors of the forearm at the lateral side of the elbow.

In continental Europe, the name lateral epicondylitis is often used for this condition, which was first described by Runge in 1873 as 'Schreibers Krampfes' (writers' cramp). In the English speaking countries, however, the condition got the name tennis elbow, which was first used by Morris in 1882 as 'lawn tennis elbow'. Actually, the name tennis elbow is not very suitable, because only few of the patients who suffer from the condition actually play tennis (Coonrad 1973). Bernhardt (1896) more appropriately described the condition as an occupational disease.

Heavy work or repetitive supination and pronation during manual labor, housework and hobbies are considered to be the most important causal factors of tennis elbow (Binder and Hazleman 1983). Little agreement exists in the literature concerning pathogenesis, pathology and natural course of the disease (Chapter I). Recent anatomical studies have produced data about the way tensile forces generated by the muscle fibers of the forearm muscles are transmitted to the bony structures of the lateral epicondyle (Van Mameren and 
Drukker 1984). This led to the concept of the Lateral Cubital Force Transmission System (LCFTS) (Chapter 2). The clinical implications of this concept for the pathogenesis of tennis elbow, however, have not yet been studied.

Several physicians regard tennis elbow as a self-limiting condition, improving with or without treatment within 8 to 12 months (Cyriax 1936, Quin and Murly 1954, Baily and Brock 1957). Other authors, however, have emphasized that a considerable group of patients shows a much longer period of tennis elbow complaints (Garden 1961, Binder and Hazleman 1983). These different opinions about the prognosis of tennis elbow may be caused by differences in the type of tennis elbow seen by these physicians.

It is known that some people with tennis elbow never seek medical attention. Those who do seek medical care will be seen primarily by their general practitioner. The orthopaedic surgeon or rheumatologist will usually see patients after the treatment by the general practitioner has failed. Thus, of the total group of persons with tennis elbow only a part will be seen by the general practitioner and an even smaller part will be treated by the orthopaedic surgeon or rheumatologist. Because of this selection it may be assumed that the treatment results of orthopaedic surgeons or rheumatologists will differ from those of general practitioners. This implies that the reported results of treatment can only be generalized to other groups of patients if it is indicated from which part of the patient population they were derived. Thus, to investigate the significance of the tennis elbow problem and its treatment, studies should be performed at all three levels (general population, general practice, out-patient department). Two studies have reported the numbers of persons with elbow complaints in the general population (Allander 1974, EPOZ 1979). Both studies did not provide information about the number of patients treated, the duration of their complaints and the referral pattern of the general practitioner.

This thesis, therefore, presents the findings of a study of the prevalence of tennis elbow in the general population. Data were gathered concerning the number of people in the general population who had been or were being treated (Chapter 3 ). At the same time the incidence of tennis elbow in general practice was studied in 19 general practices in the region of Maastricht (Chapter 3). This was done in the same period as the study in the general population, to avoid the data being influenced by seasonal fluctuations in incidence and prevalence. In addition, all patients seen for tennis elbow at the orthopaedic out-patient department were registered, as is described in Chapters 4 and 5. The data of these studies were combined to estimate the actual number of patients treated and the pattern of the general practitioner's referrals to the medical specialist. 
Most clinical studies of tennis elbow show considerable methodologic deficiencies related to the randomization process, description of the patient population and the follow-up schedule (Kamien 1990). In an analysis of 159 studies, published since 1966, Label et al. (1990) found no scientific basis for any treatment of tennis elbow. In addition, little is known about the type of treatment used by the general practitioner for tennis elbow complaints or about the results of such treatment. For that reason, we also performed a study of the types of therapeutic regimen in the general practices in our region. The one-year results of such treatments by the general practitioner were investigated (Chapter 3). The study was intended to analyze current treatment in general practice without influencing it. This inevitably led to many types of treatments with relatively small numbers of patients in each group. Consequently, this study could not provide data allowing a comparison of different types of treatment.

We were interested in the effects of different therapies. Therefore we studied the efficacy of the two most commonly used therapies for tennis elbow, physiotherapy (Cyriax) and corticosteroid injections, in a prospective randomized trial (Chapter 4).

After failure of conservative treatment the only therapeutic option left is operative treatment. Many operative techniques have been described but few have been adequately analyzed. Of these techniques lateral release of the common extensor origin is rather frequently used, because it is a limited operation which can be performed under local anaesthesia as an out-patient procedure. The results of this procedure were studied in a group of patients with resistant tennis elbow in a prospective non-randomized study (Chapter 5).

A more precise localization of lesions in tennis elbow might lead to more selective conservative and operative treatment. Therefore, we wanted to find out whether, on the basis of the anatomical concept of the LCFTS (Chapter 2), we could come to a more accurate localization of the lesion in the LCFTS in patients with a tennis elbow. This study was performed with a group of patients with tennis elbow seen at the out-patient clinic of the Department of Orthopaedics (Chapter 6).

Some cases of lateral elbow pain with local tenderness along the radial nerve are believed to be caused by compression of the radial nerve (Roles and Maudsley 1972, Werner 1979). Other authors have challenged the concept of this so-called radial tunnel syndrome as a compression neuropathy (Van Rossum et al.1978). Because of the conflicting data in the literature, we tested the hypothesis that the 
radial tunnel syndrome is a compression neuropathy. We performed a neurophysiological study in patients diagnosed on clinical grounds as having a radial tunnel syndrome (Chapter 7).

In summary, the aim of this thesis is:

- to study the number of symptomatic persons (prevalence) in the general population and the actual number of patients treated for tennis elbow.

- to study the incidence of tennis elbow in general practice as well as the way it is treated and the results of this treatment in general practice.

- to compare the results of two frequently used treatments for tennis elbow local corticosteroid injections and physiotherapy (Cyriax) - at the orthopaedic out-patient clinic.

- to study the results of operative treatment (lateral release procedure) in resistant tennis elbow.

- to study the clinical implications of the anatomical concept of the Lateral Cubital Force Transmission System with regard to the pathophysiology of tennis elbow.

- to test the hypothesis that the so-called radial tunnel syndrome is caused by a compression neuropathy of the radial nerve. 
CHAPTER

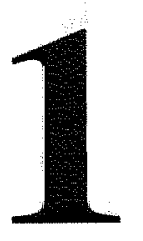

Survey of the literature 


\section{INTRODUCTION}

Tennis elbow may be defined as a condition with pain at the lateral side of the elbow which is aggravated by active extension of the wrist (Friedlander et al. 1967). It is one of the commonest lesions of the arm (Chard and Hazleman 1989). Patients with tennis elbow show rather similar symptoms and signs. Tennis elbow is, however, not believed to have one single cause. On the contrary, many different pathological entities have been proposed as causes of tennis elbow, leading to a confusing list of causes (Garden 1961). The lack of uniformity in the opinions about the cause of tennis elbow has resulted in a lack of uniformity in conservative and operative treatment. This chapter reviews the etiology, pathology and treatment, as well as the diagnosis and epidemiology of tennis elbow.

\subsection{ETIOLOGY AND PATHOLOGY}

Cyriax, reviewing the literature in 1936, listed 26 possible causes of tennis elbow and since then this list has been further extended (Cyriax 1936, 1982). The list includes bursitis, radiohumeral synovitis, synovial fringe protrusion, periostitis, osteitis, infection, neuritis, aseptic necrosis, joint and capsular lesions, muscle rupture, fibrositis, myofascitis, chronic inflammatory reaction, subtendinous tissue lesion, calcification, tendinitis, radial nerve entrapment, arthritis and cervical radiculopathy (Bernhang 1979). So far no general agreement has been reached as to the nature of the underlying pathologic changes.

One of the main reasons for this continued uncertainty about the pathology of tennis elbow is that biopsies can only be obtained in operations for chronic lesions (Chard and Hazleman 1989). Hence, little is known about the histology 
of tennis elbow with a short history. No pathological studies of the period of early symptoms have been reported (Coonrad and Hooper 1973). Moreover; there is only limited knowledge of the normal histology of structures near the lateral epicondyle and of changes caused by aging (Uhthoff and Sarkar 1980). Degenerative changes may arise in tissue without being pathological. Furthermore, long conservative treatment including physiotherapy and corticosteroid injections may obscure the original histology.

The suggested causes of tennis elbow can be classified into four groups:

I. Tendinopathy

Runge (1873) postulated periostitis to be the cause of tennis elbow and he was supported by many including Gonzette (1931) and West (1938). Osgood (1922), however, reported a case with an inflamed bursa underlying the conjoined extensor tendon, which was entirely extra-articular and without joint connection. Hughes (1950) also suggested that the pathology of tennis elbow was located in a subtendinous space between the common tendon and the capsule of the radio-humeral joint. Normally, this space is thought to be occupied by loose areolar tissue, but in tennis elbow it might be filled with fibrous tissue. Goldie (1964), who performed the first systematic study of pathology of tennis elbow, also observed reactive (granulation) tissue in the subtendinous space beneath the extensor origin, which he considered to cause symptoms of tennis elbow.

Hansson and Horwich (1930) and Hohmann (1933) thought that tennis elbow was caused by avulsion of the tendon origin. Cyriax (1936) theorized, without pathological or surgical documentation, that macroscopic or microscopic tears of the common extensor origin were the most likely cause of the symptoms. Meherin and Cooper (1950), however, could not detect any evidence of inflammatory processes or fibrosis indicating a tear of the common extensor origin. Goldie (1964) did not observe an actual tear in the common extensor tendon either. Coonrad and Hooper (1973) claimed histological support of the tendon rupture theory. In 39 operations they noticed 28 ruptures -22 superficial and 6 deep ruptures. Histology of the torn margins of tendons revealed round cell infiltration, scattered foci of fine calcification and scar tissue with marginal areas of cystic degeneration. Fibrinoid degeneration was evident in some cases. It was suggested that the lack of prior description of these lesions was attributable to a failure to expose the extensor origin at operation. Coonrad (1986) pointed out that deep ruptures may leave the superficial attachment to bone undisturbed, totally hiding the underlying pathology. The traditional release of the extensor aponeurosis as described by Hohmann (1933) and Spencer and Herndon (1953) 
does not allow an adequate visualization of the origin of the extensor carpi radialis brevis (ECRB). Using an alternative operative technique, which does expose the origin of the ECRB, Nirschl (1979) described 82 lesions of ECRB in 88 tennis elbows. Microscopically, there was angiofibroblastic hyperplasia.

Although Coonrad and Hooper (1973) and Nirschl (1979) described ruptures of the extensor origin, their reports are contradictory with respect to the localization of the ruptures. The operative technique used, the application of a tourniquet and the use of a magnification loupe may have influenced visualization of the ruptures. Although Uhthoff and Sarkar (1980) did not notice any macroscopic ruptures in the common extensor tendon, microruptures were present in almost all extensor tendon specimens. All tendon specimens showed scattered areas of thinning and fibrillation of collagen bundles. Ruptures were characterized by a break in the axial arrangement of the collagen bundles and amorphous debris was usually present at the site of the rupture. The authors concluded that mechanical strain was primarily responsible for the pathological changes, because there was an absolute lack of any morphological signs of inflammation. Chard and Hazleman (1989) could not detect evidence of inflammation in biopsies from their patients either, but instead observed mesenchymal transformation of the tendon close to its insertion, with fibrocartilage, calcification and new bone formation. In a study by Regan et al. (1990), the presence of vascular and fibroblastic proliferation differed statistically from cadaveric controls. Calcific deposits and hyaline degeneration were also significantly more often in tennis elbow than in controls. In their histological studies Doran et al. (1990) observed evidence of a repair process in response to tissue damage of the bone-tendon junction. Uhthoff and Sarkar (1980) found, in an electron- microscopic study, that the endothelial cells of the newly formed vascular channels were the source of proliferating mesenchymal cells differentiating toward tenocytes and these two processes together are supposed to represent an intrinsic healing mechanism in the tendon.

It has been suggested that most of the publications on tendinopathy are not contradictory but describe different stages of the same pathological process (Van der Beken and Joveneau 1983). Depending upon the duration, the severity of the symptoms and the localization of the lesions, there may be macroscopic or microscopic ruptures, which induce a repair reaction, sometimes involving the space beneath the conjoined tendon. During the repair process calcium phosphate or calcium apatite may precipitate in the tendon (Gerster et al. 1977). This process can be compared with McNab's theory of the origin of shoulder rotator cuff tears (MacNab 1973). The enthesis is thought to be a watershed between blood derived from muscle and that derived from bone and so is relatively avascular and prone to ischaemic stress (Niepal and Sit'aj 1979, Chard 
and Hazleman 1989). This vascular compromise may lead to an altered nutritional state, which after force overload may induce angiofibroblastic changes (Nirschl 1979, Uhthoff and Sarkar 1980, Chard and Hazleman 1989) and rupture of the vulnerable tissue (Coonrad and Hooper 1973). The development of the lesion may relate to the architecture of the tenoperiosteal junction, to age and to overuse (Binder and Halzeman 1989) such as occupational stress or strain with repeated minor trauma (Friedlander et al. 1967). The enthesis has an abundant nerve supply composed of various types of nerve endings (Goldie 1964, Niepal and Sit'aj 1979, Van der Wal 1988), which may be responsible for the pain reaction. The microscopic changes after corticosteroid injection at the lateral epicondyle have not been studied in depth.

\section{Intra-articular lesions}

\section{Synovial fringe}

Trethowan (1929) suggested synovial protrusion between radius and humerus in patients with tennis elbow and Mills $(1928,1937)$ supported this suggestion since he was able to elicit a click on manipulation. Allen (1947) observed a thicker than usual synovial fringe between the radiohumeral joint and suggested that this traumatic radiohumeral synovitis could be the cause of tennis elbow. Clarke (1988) described a series of symptomatic synovial fringes diagnosed arthroscopically causing symptoms of locking and crepitus.

\section{Annular ligament}

Stack (1949) considered fraying of the annular ligament one of the main causes of tennis elbow. Bosworth (1955) reported that the annular ligament riding upon the head of radius could provoke epicondlylar pain. He concluded that the symptoms resulted from repeated rotation of the asymmetrical radial head within the tight ligament.

\section{Chondromalacia}

Bosworth (1965) considered some cases of tennis elbow to be due to chondromalacia of the humeral capitulum or radial head. Newman and Goodfellow (1975) believed that fibrillation of the radial head was the most important 
initiating factor for the pain arising from the region of the annular ligament. In their opinion most operations for ternis elbow reduce the friction between the abnormal cartilage and the overlying degenerated annular ligament.

\section{Compression of the radial nerve}

Cases of resistant tennis elbow without paresis of extensor muscles have been suggested to be caused by compression of the radial nerve (Michelle and Krueger 1956, Sommerville 1963, Capener 1966, Roles and Maudsley 1972 , Hagert et al. 1977). Apart from the arcade of Frohse, the radial nerve is thought to be compressed by the extensor carpi radialis brevis, by fibrous bands in front of the radial head or by a recurrent radial fan of vessels (Roles and Maudsley 1972). The most frequently described compression of the radial nerve, however, is thought to be caused by the edge of the supinator muscle (Spinner 1968, Hagert et al. 1977). Roles and Maudsley (1972) and Werner (1979) have postulated that the local tenderness along the radial nerve, especially where it passes the fibrous edge of the supinator muscle, is characteristic of radial tunnel syndrome. Both syndromes, however, share several characteristics, such as lateral elbow pain which is aggravated by extension of the wrist, subjective weakness of the extensors of the forearm and radiation of the pain into the forearm (Hagert et al. 1977, Werner 1979, Jalovaara and Lindholm 1989). Neurophysiological studies in support of this hypothesis have been rare and have not provided many details (Van Rossum et al. 1978).

\section{Cervical radiculopathy}

Neuralgia due to cervical spine pathology has also been suggested as one of the causes of tennis elbow (Jones 1958, Gunn and Milbrandt 1976). Gunn and Milbrandt (1976) directed treatment at the cervical spine after at least 4 weeks of treatment of the elbow had failed. This treatment was successful in 86 per cent of patients and for that reason the authors postulated a relation between tennis elbow and disorders of the cervical spine. Nirschl (1985), however, considered osteoarthritis in the cervical spine in patients with a tennis elbow a coincidental finding. Although the lateral epicondyle is one of Smythe's tender points for diagnosing Repetitive Strain Injury Syndrome (Fibrositis), tennis elbow can be distinguished from this syndrome by physical examination (Miller and Topliss 1988, Smythe 1988). Several German authors have performed EMG studies with surface electrodes in patients with tennis elbow (Tönnis 1977, Eickhoff and Weh 1986). Tönnis (1977) found increased electrical activity in the extensor digitorum muscle at rest compared to the asymptomatic arm of patients. Weh 
and Baumann (1989) noticed that the height and integrals of anplitudes in extensor muscles during extension were considerably smaller in the diseased arms. These authors suggested that in tennis elbow the extension of the wrist is achieved by a smaller number of muscle fibers. This can be caused by a mechanical trauma of the extensor origin but may also be induced by cervical spine pathology.

\subsection{DIAGNOSIS}

\subsubsection{Complaints}

Pain starts at the outer side of the elbow. Contraction of the forearm extensors aggravates the pain at the lateral epicondyle (Goldie 1964). The pain may be sharp or dull and increases during grasping and lifting. Grip strength is often diminished because of pain. During some activities the pain may be so severe that patients drop even a light object held in the hand. The pain usually radiates into the forearm, but may also radiate into the upper arm (Cyriax 1982, Chard and Hazleman 1989). Although the pain increases during muscle activities, patients may also complain about pain at rest. Sometimes there is some stiffness of the elbow on waking (Cyriax 1982). The onset of symptoms can be sudden or gradual (Wadsworth 1987). In acute cases, patients sometimes describe a relation with a direct trauma to the lateral epicondyle. Patients with slowly increasing symptoms may notice a relation between the onset of symptoms and repetitive activity, overuse or sudden overt force (Coonrad 1986).

\subsubsection{Physical examination}

There is distinct point tenderness usually at the ventral aspect of the lateral epicondyle. A greater area around the lateral epicondyle, however, may show slight tenderness (Wilhelm and Gieseler 1964). Local swelling and redness at the lateral epicondyle have been reported (Garden 1961). Wasting of muscles is very unusual in tennis elbow. Passive and active extension of the elbow may be restricted in severe cases (Friedlander et al. 1967). The flexion is rarely limited.

Resisted movements of elbow, wrist and fingers may provoke pain at the lateral epicondyle and can be used to localize the lesion. The most frequently utilized test is resisted extension at the wrist (extensor carpi radialis brevis and longus, and extensor carpi ulnaris). Care should be taken that the elbow is held in full extension (Cyriax 1982, Wadsworth 1987). Resisted extension of the middle 
finger may also be painful (Wadsworth 1987) (Figure 1-1). Resisted pronation and supination of the forearm less often produce pain at the lateral epicondyle (De Wolf 1984). Resisted palmar flexion of the wrist is usually not painful (Spencer and Hemdon 1953).

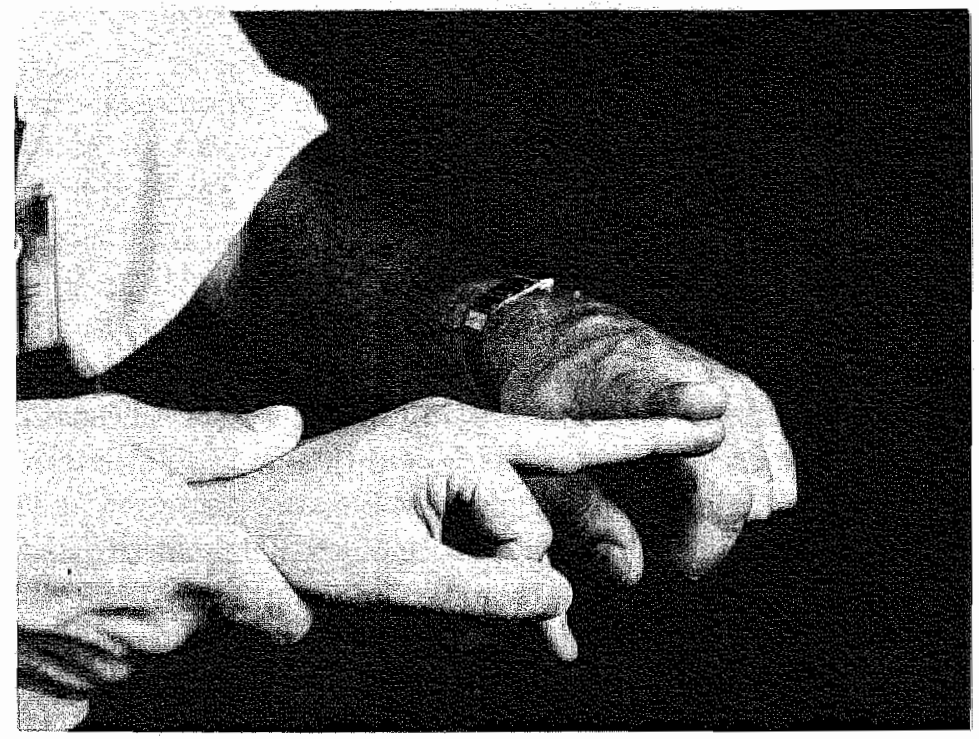

Figure 1-1.

Resisted extension of the middle finger.

In the literature many other tests have been described, such as the 'chair test' (severity of pain produced by lifting a chair with one pronated hand) or modifications of this test (lifting a book or a halter). These tests are, however, similar to resisted movement of wrist or hand. Coenen (1986) has described 'das Fingerschnips-Zeichen' (finger-snapping test). The index and middle finger must be pushed against the thumb. Then the two fingers should be extended quickly, making a movement similar to the shooting of paper balls by schoolchildren. When this test produces an acute, sharp pain, it is considered to be positive, and is a strong support for the diagnosis of a classical tennis elbow. If the test does not produce pain, however, the diagnosis of tennis elbow should be reconsidered and an arthrogenic or spondylogenic cause of the pain must be considered.

Passive stretching of some of the forearm extensor muscles may produce pain. This forced elbow extension test is carried out with a flexed elbow, the forearm in pronation and the wrist in palmar flexion. From this position the elbow is 
extended and pain will arise at the lateral epicondyle, which may limit the full extension of the elbow (Wadsworth 1987). Neurological and vascular examination generally do not show abnormalities in patients with tennis elbow.

\subsubsection{Severity of tennis elbow}

There is no generally accepted system for grading the severity of tennis elbow. The parameters most often used in clinical studies are severity of pain, tenderness and pain provoked by resisted dorsiflexion of the wrist. Because these parameters are rather subjective, some authors have used grip strength as a quantitative measure of the response to treatment (Goldie 1964, Nirschl 1979, Thurtle et al. 1984).

\subsubsection{Differential diagnosis}

Arthritis of the elbow or synovial irritation may produce pain at the lateral region of the elbow. Osteochondritis dissecans of the humeral capitulum (M.Panner) may also cause pain in this region. In these conditions palpation of the radiohumeral joint may be painful and rotation of the forearm may produce pain or crepitation of the joint (Newman and Goodfellow 1975, Wanivenhaus 1986). Elbow radiographs may be useful in diagnosing these disorders (Wanivenhaus 1986, Wadsworth 1987). Arthroscopy may be indicated in selected cases (Wadsworth 1987, Clarke 1988, Verhaar et al. 1991). Compression of cervical radices may produce brachialgia and elbow pain. An accurate neurological examination, electromyographic examination and radiographs of the cervical spine may be indicated if cervical pathology is suspected (Gunn and Milbrandt 1976).

\subsubsection{Supplementary examination}

Radiographic examination

Elbow radiographs are usually normal (Goldie 1964). Some authors, however, consider radiographs necessary to eliminate the possibility of arthritic changes at the radiohumeral articulation (Carp 1932, Wessels 1981, Wanivenhaus 1986, Wadsworth 1987).

Radiographs of the elbow may show a dense shadow distal to the lateral epicondyle in a minority of patients with tennis elbow. Schmitt (1921), Carp (1932), Hamilton (1938) and Hughes (1950) regarded these as calcium deposits in a bursa near the lateral epicondyle. Histologically, Meherin and Cooper (1950) observed slight calcium deposition in the regular dense connective tissue 
at the epicondyle, which was not found in cadavers. These calcifications are thought to be a feature of tendinous healing and are considered to be an analogue of the calcification at the insertion of the supraspinatus tendon (Hughes 1950, Cowan and Stone 1952, Uthoff et al. 1976, Niepal and Sit'aj 1979). No relation has been found between the amount of calcium present and the severity of the clinical symptoms and findings (Cowan and Stone 1952). Table 1-1 lists percentages of calcification on radiographs reported by different authors.

Table 1-1.

Calcification at the lateral epicondyle on radiographs of patients with a tennis elbow

\begin{tabular}{lrlrl}
\hline & & \multicolumn{3}{c}{ Calcification } \\
\cline { 4 - 5 } Authors & & $\begin{array}{l}\text { No of } \\
\text { X-rays }\end{array}$ & $N$ & $(\%)$ \\
\hline Gondos & 1958 & 20 & 7 & $(35)$ \\
Hohl & 1961 & 64 & 3 & $(5)$ \\
Goldie & 1964 & 95 & 26 & $(27)$ \\
Ilfeld and Field & 1966 & 45 & 9 & $(20)$ \\
Baumgard & 1982 & 34 & 4 & $(12)$ \\
Waldis & 1989 & 39 & 7 & $(18)$ \\
\hline
\end{tabular}

O'Neil et al. (1980) were unable to substantiate the hypothesis of Nirschl (1973) that a prominent radial head bowstringing the common extensors in pronation might be an important factor in the pathogenesis of tennis elbow. In a study comparing the radiographs of patients suffering from tennis elbow with those of controls no differences in the shape of the radial head were found. Freund et al. (1970) did not find ulna minus more often than in controls.

\section{Electromyographic studies}

Electromyographic studies are only indicated if cervical radiculopathy or pathology of the brachial plexus or nerves of the upper extremity is suspected. 


\section{Thermography}

Shilo et al. (1976) and Binder et al. (1983) performed thermographic studies in patients with tennis elbow. Thermography of the affected elbow showed a discrete localized area of increased heat near the lateral epicondyle ("hot spot") in a high percentage of patients. Analysis of the gradient across the abnormal area showed good correlation with clinical severity of the condition (Binder et al. 1983). Thirty per cent of patients also had a hot spot near the lateral epicondyle in the unaffected side (Binder et al. 1983).

\section{Echography}

Commandre and Valdener (1983) have described one patient with a rupture of the muscle fibers of the extensor carpi radialis longus and brevis diagnosed by means of echography.

\section{Scintigraphy}

Koppers and Riel (1982) examined 10 patients with a tennis elbow by threephase scintigraphy using ${ }^{99 \mathrm{~m}} \mathrm{Tc}-\mathrm{MDP}$. There was no uptake during the perfusion and soft tissue phases. In the late phase, there was focal bone uptake in one patient.

\section{MRI}

The value of MRI in diagnosis and localization of the lesion has not yet been evaluated. No data about the number of abnormalities found with MRI are available.

\subsection{EPIDEMIOLOGY}

\subsubsection{General population}

Knowledge about the annual incidence and prevalence of tennis elbow in the general population is rather limited. Allander (1974) found a prevalence between 1 and 3\%, depending upon age. The annual incidence of tennis elbow in this $\mathrm{S}$ wedish study was less than $1 \%$. The prevalence rate of tennis elbow in the 
Dutch general population is not well known. The Dutch EPOZ study in Zoetermeer reported a prevalence of elbow complaints of $2 \%$ among men and $4 \%$ arnong women (EPOZ 1979).

Among 7600 workers, Kivi (1982) found an incidence of tennis elbow of $0.6 \%$. The main cause of tennis elbow was over-exertion of the finger and wrist extensors in trained workers (Luopajärvi et al. 1979, Roto and Kivi 1984). A study by Sinclair (1965) showed that particularly repetitive grip movements as well as repetitive supination and pronation were causative factors for tennis elbow.

In tennis players the incidence and prevalence of tennis elbow is high. In a study of 532 tennis players Gruchow and Pelletier (1979) noticed an incidence of $9 \%$ and a prevalence of $14 \%$. Moreover, $25 \%$ of the tennis players had experienced a period of lateral elbow pain in the past. Priest (1976) found a prevalence of $28 \%$ among 231 tennis players. The incidence increased with the age of the player and the amount of daily playing time (Priest 1976, Kitai et al. 1986). The racket used (racket frame, string tension, racket handle) may also play a role in the etiology of tennis elbow (Nirschl 1970, Colt 1970, Leach and Miller 1987, Wadsworth 1987). However, no clinical studies are available to substantiate these assumptions. Despite the high incidence among tennis players, however, tennis elbow has more often been met in clinical practice in non-athletes than in athletes (Coonrad and Hooper 1973, Binder and Hazleman 1983).

In general practice, incidence figures of tennis elbow are much lower than those found in the general population. In the Netherlands, Lamberts (1975) and Blanken (1981) reported an incidence of 5 per 1000 patients. Van Lisdonk et al. (1990) reported an incidence of 6.6 for men and 6.4 for women per 1000 patients. In Great Britain, Hamilton (1986) noted an incidence rate of 4.2 per 1000 patients.

\subsubsection{Predilection factors}

Some predilection factors have been described:

- In the general population prevalence of tennis elbow seems to be higher in females than in males (Allander 1979, EPOZ 1979). In clinical studies, however, the sex distribution has not proved similar to these epidemiological surveys. Hohl (1961), Lamphier et al. (1959) and Kurppa et al. (1979) reported a predominance of males among the patients. An equal distribution for men 
and women was reported in the series of Spencer and Herndon (1953), Bosworth (1965), Coonrad and Hooper (1973), Nirschl and Pettrone (1979), Calvert et al. (1985).

- Tennis elbow usually affects patients between 30 and 60 years of age. The highest numbers of patients are found among people in their fourth decade (Nirschl 1985).

- The dominant hand is affected in most patients (Wadsworth 1987, Chard and Halzeman 1989).

- It has been suggested that the increased risk of developing a bilateral tennis elbow is caused by greater stress on the non-painful elbow. However, there may also be an intrinsic factor.

- The higher incidence of other soft tissue diseases (carpal tunnel syndrome, impingement syndrome etc.) has not been sufficiently explained (Nirschl and Pettrone 1973, Boyd and Mcleod 1973, Murray-Leslie and Wright 1976, Day 1978, Binder et al. 1983). Nirschl (1985) suggested a constitutional factor to be responsible for a predisposition to develop tendinitis at different locations.

\subsubsection{Economic aspects}

Tennis elbow may lead to sick leave, disablement pension, transfer to lower-paid jobs, and even early retirement (Dimberg 1987). However, few studies have reported these economic consequences. Schonk (1985) reported that 1.2 pro mille of the sick leave days in the Netherlands was caused by tennis elbow. In 19843148 people received a disablement pension (WAO) as a result of tennis elbow (Van der Valk 1987). 512 persons received this pension for the first time, while this compensation was terminated in 189 people in that year.

\subsection{CONSERVATIVE TREATMENT}

There is little agreement about the etiology of tennis elbow, and the same controversy exists about conservative treatment. Many treatment modalities have been used for the complaint.

Reduced activity may result in resolution of symptoms and for that reason some authors consider tennis elbow a self-limiting condition leading to full recovery in a period of 8 to 12 months (Cyriax 1936, Quin and Binks 1954, Baily and Brock 1957). Binder and Hazleman (1983), however, found that 40 per cent of their patients had persisting or recurrent discomfort on use at review 1 to 5 years after initial presentation at the out-patient clinic. Only 40 per cent of the patients 
with tennis elbow treated by Gerberich and Priest (1985) recovered completely. Duration of pain prior to treatment was identified as an important variable in the success of treatment. Gerberich and Priest (1985) identified the degree of pain prior to treatment as the most important predictor of recovery; the greater the pain, the more likely was a complete recovery. These authors suggested that their findings illustrated the need for early case-finding to elicit the most favourable outcome in treatment.

In a review Friedlander et al. (1967) described 43 different conservative treatment modalities, including radiotherapy (Cowan and Stone 1952, Meherin and Cooper 1950 ), manipulation (Mills 1928), rest and immobilization with a cockup splint (Hansson 1930, Lambrecht 1948), local injections, drugs and physiotherapy. Afterwards the beneficial effects of acupuncture (Brattberg 1983) and braces (Ilfeld and Field 1966, Froimson 1971, Nirschl 1973) on the tennis elbow have been described. These braces were designed to diminish the 'overload' forces (Figure 1-2).

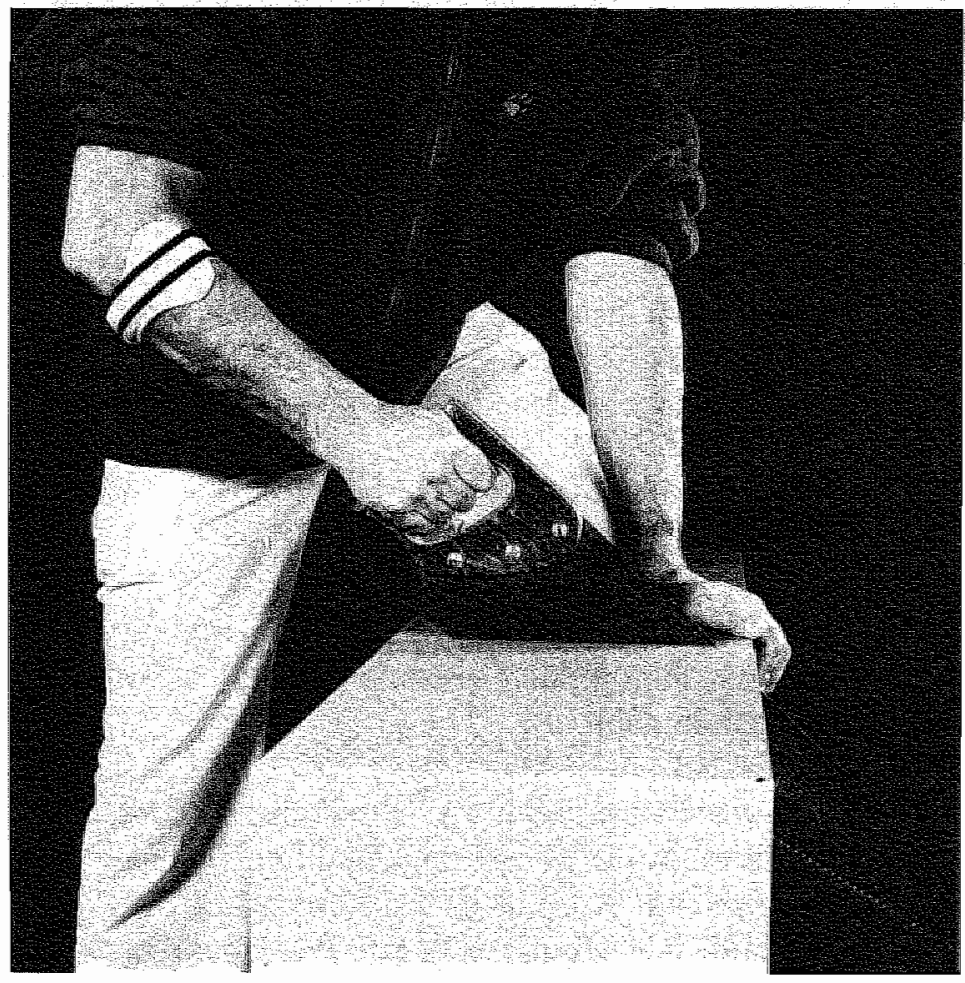

Figure 1-2.

An example of one of the braces designed to diminish the 'overload' forces. 
Binding the muscles might limit expansion, which decreases the contribution to force production generated by muscle fibers proximal to the band (SnyderMackler and Epler 1989). The brace designed by Iffeld and Field limits full extension and rotation of the elbow. In contrast to European practices exercises to strengthen the forearm muscles are often prescribed in the USA (Nirschl 1973). Trethowan (1986) emphasized that ergonomic appraisal of the work, which caused the tennis elbow may also be an important aspect of the conservative treatment of tennis elbow.

In the Netherlands, the most frequently used conservative modalities of treatment are anti-inflammatory drugs, physiotherapy and injection of corticosteroid into the tender site at the lateral epicondyle. $2.7 \%$ of the patients referred by the general practitioner for physiotherapy are treated for tennis elbow (Groenewegen 1990). Annually, the Dutch general practitioners refer 81 per 1000 patients for physiotherapy (Groenewegen 1990). Accurate data about the numbers of corticosteroid injections for tennis elbow in general practice are not available.

\subsubsection{Injections}

After the discovery of the effects of hydrocortisone on rheumatic diseases in 1949, Hollander (1953) was the first author to report the use of local injections of hydrocortisone in patients with tennis elbow. The injections were successful in 22 out of 25 patients. Shortly after this publication other small series were described, which confirmed the effectiveness of the local corticosteroid injection (Figure 1-3).

In prospective randomized studies, corticosteroids proved to be superior to other injection solutions such as procaine, xylocain and saline (Bailey and Brock 1957, Day et al. 1978). Hence, the action is not based on mechanical distension of tissues by fluid injection (Bailey and Brock 1957, Day et al. 1978). Only one study has compared the effect of two different corticosteroids in tennis elbow (Clarke and Woodland 1975). No significant differences were found. Rather high recurrence rates after injection of corticosteroids have been described (Freeland and Gribble 1954, Quin and Binks 1954, Murley 1954, Young et al. 1954, Lamphier 1959), especially in studies with a follow-up of six months or more (Clarke and Woodland 1975, Nevelös 1980). Transcutaneous injections using high pressure proved to be as effective as needle injections of hydrocortisone acetate in patients with tennis elbow (Hughes and Currey 1969). Some authors have mentioned the successful use of corticosteroids in combination with manipulation (Hohl 1961). Injection solutions such as histamine and 


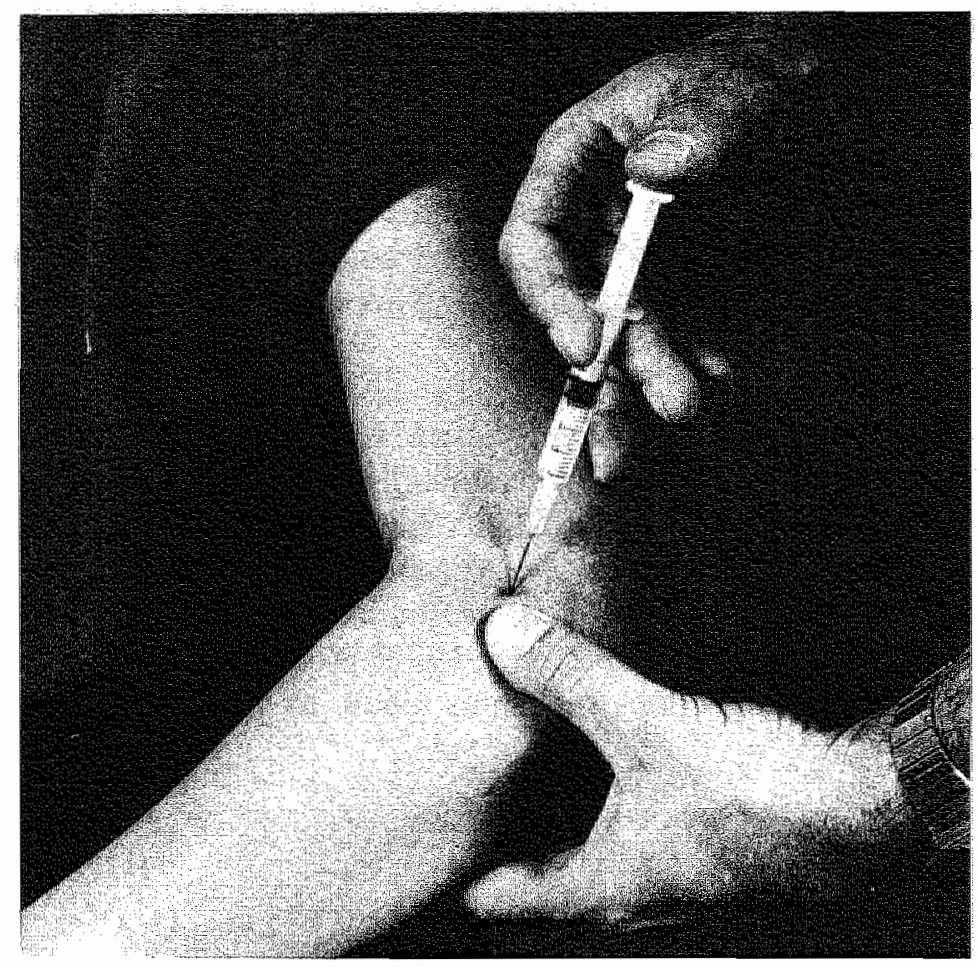

Figure 1-3.

Corticosteroid injection given at the common origin of the forearm extensors at the epicondyle

orgotein (which has an anti-inflammatory action based on the destruction of oxygen radicals) have also been used in tennis elbow (Kuipers 1943, Miller and Moll 1983). Although a double blind clinical trial seemed to prove orgotein to be more effective than procaine injections in tennis elbow that had existed for less than 6 weeks, orgotein did not prove to be more effective than placebo therapy in tennis elbow that had existed for more than 6 weeks. Saartok (1986) reported no differences after comparing the effectiveness of naproxen with that of corticosteroid injections, but his observation period was just two weeks.

There is no agreement in the literature about the most effective corticosteroid preparation for tennis elbow, nor about the dose or the injection technique (Holden and Hume Kendall 1961). Although the effectiveness of corticosteroid injection is well-established, corticosteroid injections may have significant sideeffects. 


\section{Side-effects of corticosteroid injections}

\section{I-Ruptures}

Local injections around the achilles tendon, rotator cuff, biceps tendon and the patellar tendon have resulted in ruptures of these tendons in some patients (Krahl and Plaue 1971). Therefore, some authors caution against the use of corticosteroid injection in over-use disorders (Krahl and Langhoff 1971, Hermans 1977). Other authors, however, consider these tendon ruptures a rare complication of corticosteroid injections (Gray and Gottlieb 1983). Moreover, within the first one or two weeks, corticosteroids induce a relatively rapid increase in the tensile strength of the collagenous tissue. This is followed by a progressive reduction in the thickness and number of collagen bundles, mainly caused by inhibition of collagen synthesis (Carlstedt 1987), which may cause rupture of the tendon. As a result of these two different effects of corticosteroids the strength of the tendons has been found not to be reduced, even though the collagen content was reduced (Carlstedt 1987). This does, however, depended upon the number of injections given and the dose of the corticosteroid solution.

\section{2-Increased pain and swelling}

Shortly after the injection a temporary increase in pain and swelling may be noticed (Lamphier 1959, Ilfeld 1966). The frequency of this side-effect is not well known. The reaction may persist for 48 hours and is considered to be an inflammatory response to crystals in the corticosteroid preparation (McCarty and Hogan 1964).

\section{3-Infection}

Iatrogenic joint or soft tissue sepsis is a rare complication of corticosteroid injection (Gray and Gottlieb 1983).

\section{4-Skin atrophy}

Leakage of corticosteroid material along a needle track, or improper needle placement, may cause subcutaneous fat necrosis or skin atrophy. An area of thin, depressed, or hypopigmented skin may be a permanent result, although these changes are often reversible (Fisherman et al. 1962, Penners et al. 1977, Bentley 1981) (Figure 1-4).

\section{5-Other adverse reactions}

Transient weakness of an injected extremity, nausea, dysphoria, or hypersensitivity reactions to the corticosteroids, steroid vehicle or local anaesthetic have occasionally been observed (Gray and Gottlieb 1983). 


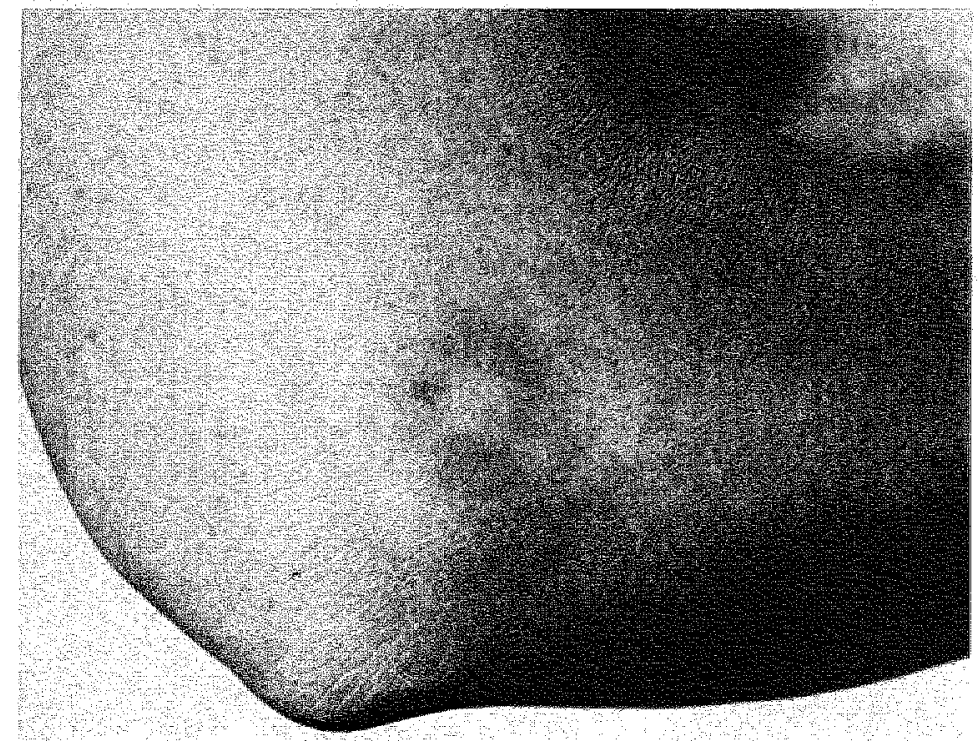

\section{Figure 1-4.}

The skin over the lateral epicondyle is thin, depressed and hypopigmented due to leakage of corticosteroid material along a needle track. improper needle placement or high doses.

\subsubsection{Physiotherapy}

The effectiveness of physiotherapy in tennis elbow has been less extensively studied than the treatment with corticosteroid injections.

In a combined prospective and retrospective study, Binder et al. (1985) found 12 ultrasound treatments in a 4 to 6 week period more effective than placebo therapy. This study was, however, methodologically less than perfect, since the data of the retrospectively seen and prospectively followed patients were combined. Lundeberg et al. (1988) did not observe any differences between ultrasound treatment and placebo treatment in tennis elbow. However, these authors did observe a statistically significant difference between the group of patients receiving placebo ultrasound (= non-functioning apparatus) and a group of patients who were only treated by prescribing rest. The authors assumed that this beneficial effect of placebo ultrasound treatment was based on careful supervision or on the friction of the head of the ultrasound machine on the lateral epicondyle. Lundeberg et al. (1988) noticed that vibration at the lateral epicondyle did result in a temporarily increased threshold to pain in the lateral 
epicondyle, which was not observed in control persons. Neither Devereaux et al. (1985) nor Chard and Hazleman (1988) observed any difference between electromagnetic treatment of tennis elbow and placebo treatment. Lundeberg et al (1987) concluded that laser treatment was not significantly better than placebo in the treatment of tennis elbow.

Thus, with the exception of Binder et al. (1985), who observed a beneficial effect of intermittent ultrasound treatment, the literature does not provide any data suggesting that physiotherapy produces better results than placebo treatment. 'Spontaneous' recovery in studies with a control group varied from $29 \%$ (Binder et al. 1985) to 53\% (Devereaux et al. 1985).

Cyriax (1936 and 1982), nevertheless, claimed good results with a specific physiotherapeutic regimen consisting of deep transverse friction and Mills' manipulation. Deep transwerse frictions are a kind of local massage, with the tip of the thumb on the front of the lateral epicondyle. These frictions are claimed to produce reactive hyperaemia at the scar, acting as an analgesic. It also is supposed to soften the tenoperiosteal junction. This massage alone is regarded as of minor value and is the preliminary to the Mills' manipulation (Cyriax 1982, De Bruyn 1984) (Figure 1-5). In this manipulation the elbow is flexed, with the forearm in pronation and the wrist in palmar flexion. From this position the elbow is forcefully extended. This manipulation may produce much pain at its performance, which may be limited by the deep friction. Cyriax (1982) reported that four to twelve sessions are required. The Mills' manipulation is intended to pull apart the two surfaces of connective tissue joined by a painful scar. If full separation is attained that way, it results in permanent lengthening of that part of the common extensor origin. Injections with local anaesthetic are recommended for accurate localization of the lesion in the extensor origin. Cyriax and Troisier (1953) emphasized, that this technique can only be performed by specially trained physiotherapists. Corticosteroid injection may replace the sometimes painful deep transverse friction, but Cyriax and Troisier (1953) considered the manipulation the essential part of treatment.

Although the orthopaedic medicine of Cyriax has found wide and increasing application in the Netherlands, little is known about the effectiveness of this treatment compared with any other modalities of treatment (Jonquière 1986). Jonquière did not observe any differences in the results of treatment of 'ordinary' physiotherapy and the treatment advised by Cyriax. 


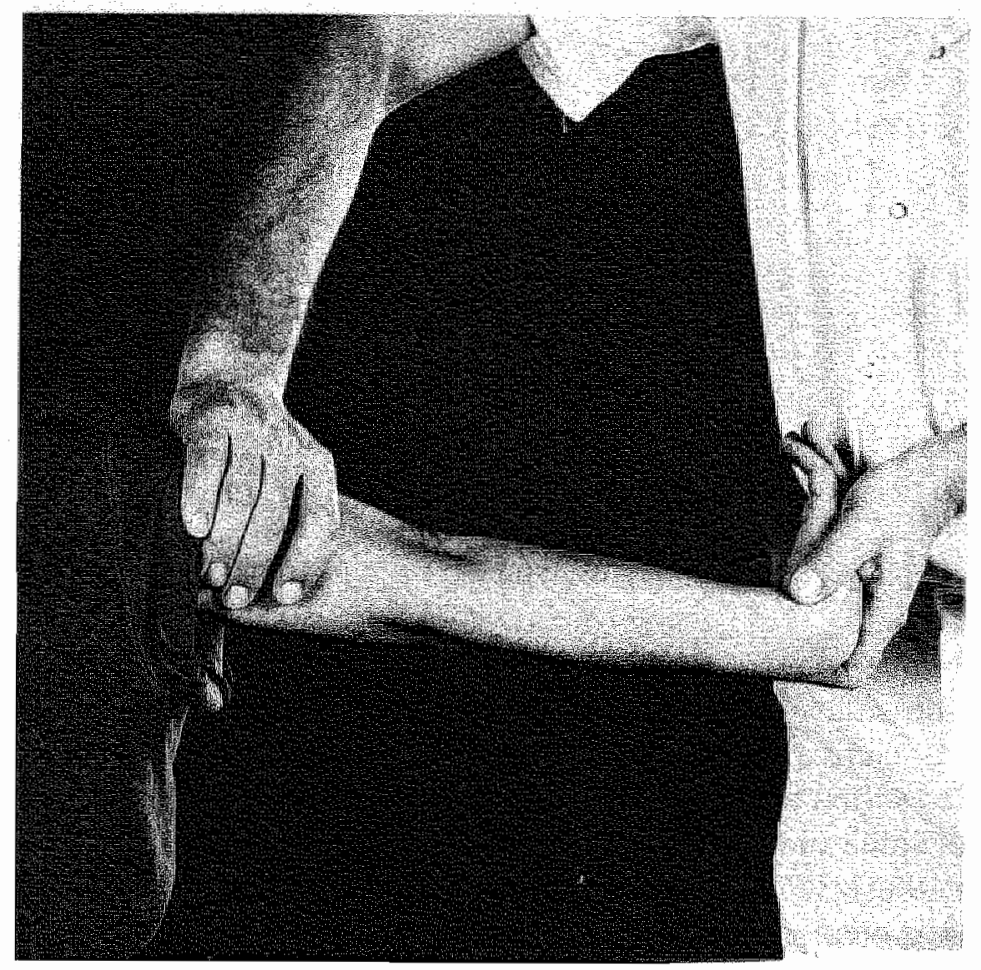

\section{Figure 1-5.}

Mills' manipulation. In this manipulation the elbow is flexed, with the forearm in pronation and the wrist in palmar flexion. From this position the elbow is forcefully extended.

\subsubsection{Manipulations}

In the UK in particular, Mills' manipulation is also used for the treatment of tennis elbow without application of the principles of Cyriax. These manipulations have their supporters (Marlin 1930, Cyriax 1936, Wadsworth 1987) and opponents (Meherin 1950). Wadsworth (1987) used the method of Mills with the patients fully relaxed under general anaesthesia. The author describes that in typical cases he heard a clearly audible snap as full extension of the elbow was achieved, which may be attributed to a closed lateral release or to breakdown of adhesions. Good results are claimed without further data being provided. 


\subsubsection{Operative techniques}

A clear definition of the pathological process is essential for the development of any well-conceived operation. The etiology of tennis elbow, however, is still in debate and for that reason, many operative techniques have been described. Most procedures are modifications of techniques already described in the beginning of this century. Table 1-2 presents a historical survey of the operative treatment of tennis elbow.

\section{Table 1-2.}

Historical survey of operative treatment of tennis elbow

$\begin{array}{lll}\text { Cauterization of the tender area } & \text { Runge } & 1873 \\ \text { Excision of the radiohumeral bursa } & \text { Osgood } & 1922 \\ \text { Fasciotomy of the forearm extensors } & \text { Fischer } & 1923 \\ \text { Division of the extensor origin } & \text { Hohmann } & 1926 \\ \text { Excision of the synovial fringe } & \text { Trethowan } & 1929 \\ \text { Exploration of the subtendinous space } & \text { Hughes } & 1950 \\ \text { Resection of the annular ligament } & \text { Bosworth } & 1955 \\ \text { Removal of calcium deposits } & \text { Van Demark } & 1956 \\ \text { Denervation of the radiohumeral joint } & \text { Kaplan } & 1959 \\ \text { Lengthening of the ECRB tendon } & \text { Garden } & 1961 \\ \text { Excision of scar tissue in the subtendinous space } & \text { Goldie } & 1964 \\ \text { Release of the radial nerve } & \text { Roles } & 1972\end{array}$

The various operations for tennis elbow may be classified into five groups:

\section{I- $\quad$ Release of extensor origin}

Tension on the common extensor origin is relieved. This procedure has also been described as "muscle slide procedure". Relief of tension can be achieved by 3 methods: 


\section{Fasciotomy}

Michelle and Kueger (1956) used a transverse, 4 centimeter long incision two fingerbreadths distal to the lateral epicondyle over the proximal muscle belly of the extensors of the forearm, including the intermuscular septa.

\section{2-Release of the common extensor origin from the lateral epicondyle}

A-Open procedure. Under local anaesthesia Hohmann (1933) made a $3 \mathrm{~cm}$ longitudinal incision over the epicondyle and incised the origin of the extensor carpi radialis brevis transversely just ventral to the lateral epicondyle. The limited release was only performed over the ventral part of the epicondyle.

Spencer and Herndon (1953) modified the Hohmann technique. They performed the release of the origin by stripping the entire origin, beginning at the tip of the lateral epicondyle, until the synovium had been reached. The muscles were permitted to retract. In cases with a flexion contracture Cabot (1987) added a release of the anterolateral articular capsule to the release of the extensor origin. B-Percutaneous tenofasciotomy. Baumgard and Schwartz (1982) transected the common extensor origin percutaneously, leaving a gap in the tendon of approximately $1 \mathrm{~cm}$ overlying the anterior aspect of the lateral epicondyle. A similar technique has been described by Yerger and Turner (1985).

\section{3-Lengthening the exterisor carpi radialis brevis tendon using a Z-plasty}

A-Proximally. An operative lengthening of the common extensor origin has been described by Van der Beken and Joveneau (1983). The origin was incised with a $\mathrm{Z}$-incision and a transposition was carried out, resulting in a lengthening of the extensor origin.

B-Distally. Based on the assumption that the pathology in tennis elbow is localized in the origin of the extensor carpi radialis brevis, Garden (1961) performed an open lengthening of the tendon of the extensor carpi radialis brevis just proximal to the point at which the thumb extensors cross the radius obliquely. Litt and Albassir (1983) performed lengthening of extensor carpi radialis brevis as well as extensor carpi radialis longus.

\section{II- Excision of the rupture in the extensor origin}

At operation, the extensor origin is repaired after the excision of torn tendon or granulation tissue. Additionally, some authors remove part of the lateral epicondyle.

Osgood (1922) excised a bursa lying beneath the origin of the extensors in the treatment of tennis elbow. Instead of such a bursa, Goldie (1964) found granulation tissue in this area. This granulation tissue, from the subtendinous space lying under the extensor origin, was removed. After excision, the longitudinally 
incised origin was repaired. Gardner (1970) used an inverted U-shape incision in the common extensor origin, including capsule and lateral collateral ligament, to free the lateral epicondyle. An osteotome was used to remove the epicondyle down to the cancellous bone. The flap of the extensor origin was then sutured back to the epicondyle. Preoperatively, Coonrad (1986) inserted a needle at the point of maximal tenderness at the lateral epicondyle to identify a possible lesion in the extensor origin. If a tear or scar tissue was detected, this was excised. If no scar tissue or tearing was identified, a resection of the part of the extensor origin in which the needle had been inserted was performed. Nirschl and Pettrone (1979) made an incision at the interface of extensor carpi radialis longus and the extensor aponeurosis to expose the origin of the extensor carpi radialis brevis. Pathologic tissue was excised, which generally included the entire origin of this muscle. After this resection, small holes were drilled through the cortical bone to enhance the blood supply to the tissue defect created by the excision. Froimson (1982) incised the common extensor tendon longitudinally and dissected off the origin of the lateral epicondyle over approximately $0.5 \mathrm{~cm}$ both anteriorly and posteriorly. Tears in the tendon were identified and excised, along with any granulation tissue. Subsequently, the tip of the lateral epicondyle was excised, producing a raw cancellous bone surface and permitting approximation of the debrided tendon without tension.

\section{III- Denervation}

Denervation of the lateral epicondyle is achieved operatively by severing the sensory fibers of the radial nerve innervating the lateral epicondyle.

After an anatomical study of the sensory innervation of this region Kaplan (1959) used these data for the successful treatment of three patients with tennis elbow. Wilhelm and Giesler (1962) developed an operative technique which is supposed to lead to sensory denervation of the lateral region of the elbow as well as release of the extensor origin. An extensive release of the origin is carried out and the epicondyle is denervated by cauterization.

\section{IV- Intra-articular procedures}

The radiohumeral joint is opened and an intra-articular procedure for lateral elbow pain diagnosed as tennis elbow is performed. These procedures may include partial or complete division of the annular ligament, synovectomy or debridement of the radial head, and are sometimes combined with one of the procedures from the categories I or II. 
Arthrotomy with excision of the synovial fringe between the radial head and the humeral capitulum has been recommended by several authors (Threthowan 1929, Moore 1952, De Goes and Silva 1960, Decaix 1982). Bosworth (1955 and 1965) postulated that the annular ligament was the causative factor for tennis elbow and hence excised this ligament. Boyd and Mcleod (1973) modified Bosworth's technique, because they found that excision of the annular ligament led to instability in the radiohumeral joint. They detached the extensor origin and reflected it carefully from the annular ligament by sharp dissection. Then they excised circumferentially a segment of the annular ligament, leaving the distal three-fourths of the annular ligament intact. Newman and Goodfellow (1975) performed a lateral arthrotomy in patients with a chronic tennis elbow. They found a high incidence of cartilage lesions of the radial head, which they treated successfully with debridement. Additionally, they removed the annular ligament. Bowden (1978) performed an extensive lateral release with synovectomy of the radiohumeral joint and reattachment of the extensor origin.

\section{Decompression of the radial nerve}

The radial nerve is exposed and transverse veins divided. The posterior interosseous nerve is followed distally and the fibrous edge of the supinator (Arcade of Frohse) is divided longitudinally.

\section{I- Anterior approach}

Roles and Maudsley (1972) used an anterior longitudinal approach over the ventral surface of the forearm, distal to the elbow crease and extending over the radial head. The deep fascia was divided and the brachioradialis muscle was exposed and retracted in radial direction.

\section{2- Posterior approach}

Werner (1979) employed a straight dorso-lateral incision with dissection between the extensor carpi radialis longus and brevis muscles, which gave easy access to the nerve. The nerve was decompressed by a 1 to $2 \mathrm{~cm}$ longitudinal incision through the supinator muscle.

\subsubsection{Results of operative treatment}

Reviewing the results of treatment is complicated by many factors. The most important problem is the lack of uniformity in the assessment of the results of treatment. This assessment differs considerably in the various publications about operative treatment. The criteria listed in table 1-3 are based on the criteria used 
Table 1-3.

Criteria for results assessment after treatment of tennis elbow

$\begin{array}{ll}\text { Excellent } & \text { Complete relief of pain } \\ \text { Good } & \text { Occasional slight pain after strenuous activities } \\ \text { Moderate } & \text { Improved; essentially no pain at night and slight pain at rest: } \\ & \text { discomfort after strenuous activities at a more tolerable level } \\ \text { Poor } & \text { No improvement }\end{array}$

by Roles and Maudsley (1972), Hagert et al. (1978), Nirschl and Pettrone (1979), Werner (1979), Meine (1981), Litt and Albassir (1983). Some authors also include resumption of labor as a criterium (Litt and Albassir 1983). In general, the criteria for poor or fair are rather constant in the literature. The criteria for excellent or good are less uniform.

On the basis of the criteria of table 1-3 we have summarized our review of the results of operative treatment in table 1-4. In this table excellent and good have been combined into one criterium. All studies are retrospective and many studies do not provide information about the number of patients with adequate followup. Some studies mention the number of patients seen at follow-up but do not give data about the size of the original group. The duration of the follow-up is presented in the right most column of table 1-4.

Only three studies reported a percentage of good results below 60 per cent (Caroll and Jorgensen 1968, Paar et al. 1978, Nollen 1981). Seventeen of the 37 studies describe more than 90 per cent good results. The differences are difficult to explain. Interestingly, all the less successful studies used a lateral release procedure. From table 1-4 it may be concluded that most authors claim fairly good results with very different operative techniques.

\subsubsection{Complications}

Unfortunately, only a limited number of publications give data about postoperative complications. The cumulative data of 9 series allow a total complication rate of $3.6 \%$ to be calculated; $1.6 \%$ infections or synovial fistula, $1.4 \%$ wound healing problems or hematoma and $0.6 \%$ restriction of function of the elbow joint, usually mostly a limitation of extension (Table 1-5). Although infection may result in septic arthritis, this complication has not often been seen. The data from the literature suggest a rather low complication rate. 
Table 1 m.

Results of operative treatment of tennis elbow in the literature

\begin{tabular}{|c|c|c|c|c|c|c|c|c|c|}
\hline \multirow[b]{2}{*}{ Author } & \multirow[b]{2}{*}{ Year } & \multirow{2}{*}{$\begin{array}{l}\text { Op- } \\
\text { type }\end{array}$} & \multirow[b]{2}{*}{$\mathrm{N} \sim \mathrm{Op}$} & \multirow[b]{2}{*}{ N-Fu } & \multicolumn{3}{|c|}{ Results as percentages } & \multicolumn{2}{|c|}{ Follow-up } \\
\hline & & & & & Good & Failr & Poor & Mean & $(\min , \max )$ \\
\hline Michelle & 1956 & 1 & 3 & - & 100 & 0 & 0 & - & \\
\hline Wiesner & 1952 & II & 10 & 10 & 100 & 0 & 0 & - & \\
\hline Spencet & 1953 & II & - & 49 & 95 & 0 & 5 & 13 & $(-120)$ \\
\hline Thơrmer & 1957 & II & - & 76 & 99 & 1 & 0 & - & $(24,96)$ \\
\hline Garden & 1961 & III & 50 & 50 & 100 & 0 & 0 & 1.08 & $(-,-)$ \\
\hline Caroll & 1968 & III & 16 & 16 & 19 & 50 & 31 & 26 & $(03,60)$ \\
\hline Posch & 1978 & II & 67 & 35 & 88 & 5 & 7 & 96 & $(-,-\infty)$ \\
\hline Paar & 1978 & III & - & 30 & 43 & 0 & 57 & 26 & $(04,48)$ \\
\hline Stovell & 1979 & III & 21 & 18 & 88 & 6 & 6 & 72 & $(12,168)$ \\
\hline Kammer & 1981 & III & 100 & 100 & 90 & 0 & 10 & 18 & $(03,84)$ \\
\hline Nollen & 1981 & II & 206 & 168 & 54 & 21 & 25 & 48 & $(-,-)$, \\
\hline Bengert: & 1982 & III & 79 & 63 & 94 & 3 & 3 & - & \\
\hline Schirmer & 1982 & II & 57 & 33 & 73 & 0 & 27 & - & $(03,108)$ \\
\hline Baumgard & 1982 & III & 37 & 37 & 92 & 0 & 8 & 35 & $(14,81)$ \\
\hline Litt & 1983 & III & - & 19 & 78 & 11 & 11 & - & $(24,168)$ \\
\hline VdBeken & 1983 & II & - & 34 & 91 & 9 & 0 & - & $(12,84)$ \\
\hline Calvert & 1985 & II & 42 & 37 & 89 & 0 & 11 & 74 & $(-,-)$, \\
\hline Yerger & 1985 & II & 149 & 109 & 94 & 2 & 4 & - & $(12,132)$ \\
\hline Waldis & 1989 & II & 101 & 63 & 80 & 5 & 15 & 20 & $(02,108)$ \\
\hline Coonrad & 1973 & III & - & 39 & 77 & 23 & 0 & - & $(12,108)$ \\
\hline Nirschl & 1979 & III & 88 & 76 & 85 & 13 & 2 & - & \\
\hline Kaplan & 1959 & IV & 3 & 3 & 100 & 0 & 0 & - & \\
\hline Wilhelm & 1962 & IV & - & 6 & 100 & 0 & 0 & - & \\
\hline Bosworth & 1965 & IV & 28 & 21 & 91 & 0 & 9 & - & $(12,108)$ \\
\hline Gardner & 1970 & IV & 9 & 9 & 100 & 0 & 0 & 14 & $(-, \cdots)$ \\
\hline Savastano & 1972 & IV & 20 & 16 & 75 & 25 & 0 & - & $(18,66)$ \\
\hline Boyd & 1973 & IV & 28 & 25 & 88 & 4 & 8 & - & $(05,192)$ \\
\hline Bowden & 1978 & IV & $\cdots$ & 11 & 100 & 0 & 0 & - & $(03,18)$ \\
\hline Patar & 1978 & IV & - & 40 & 80 & 10 & 10 & - & \\
\hline O'Neil & 1980 & IV & 50 & 42 & 88 & 7 & 5 & - & $(36,84)$ \\
\hline Meine & 1981 & IV & 63 & 62 & 94 & 5 & 1 & - & $(08,70)$ \\
\hline Cabot & 1987 & IV & 47 & 47 & 87 & 13 & 0 & 38 & $(22,62)$ \\
\hline Wanivenhaus & 1988 & IV & 48 & 45 & 93 & 0 & 7 & 78 & $(-,-)$ \\
\hline Goldie & 1964 & $\mathrm{~V}$ & 114 & 97 & 80 & 16 & 4 & - & $(06,36)$ \\
\hline Oerke & 1977 & $\mathrm{~V}$ & 671 & 488 & 80 & 0 & 20 & 132 & $(07,240)$ \\
\hline Kaiser & 1982 & $\mathrm{~V}$ & - & 55 & 67 & 0 & 33 & - & $(24,60)$ \\
\hline Saillant & 1989 & $\mathrm{~V}$ & 123 & 123 & 91 & 7 & 2 & 9 & $(-,-)$ \\
\hline
\end{tabular}


Legend table 1-4: Op-type (Type of operations): I Fasciotomy: II Release of extensor origin; II Excision of the rupture or the scar in the extensor origin and reconstruction of the origin: IV Denervation of the lateral epicondyle; V Intra-articular operation or combination of different types of operations; N-Op: Number of elbows operated ( $\angle$ indicates no numbers given by author); $\mathrm{N}$-Fu: Number of elbows at follow-up (- indicates no numbers given by author); Follow-up: Mean follow-up and range (minimum and maximum) in months after operation (-indicaltes absence of data).

Table 1-5.

Complications of operative treatment of tennis elbow

Types of complications

\begin{tabular}{lrrrrrrrr} 
Author & Year & N-Op & A & B & C & D & E & F \\
\hline Spencer & 1953 & 49 & 2 & - & - & - & - & - \\
Oerke & 1977 & 488 & - & 4 & - & 6 & - & $\ldots$ \\
Posch & 1978 & 42 & - & 2 & - & - & - & - \\
Nirschl & 1979 & 88 & - & - & - & 1 & 2 & $\ldots$ \\
Kammer & 1981 & 100 & - & - & 1 & - & - & 1 \\
Meine & 1981 & 63 & - & - & 4 & 1 & - & - \\
Baumgard & 1982 & 37 & - & - & - & - & - & - \\
Vd Beken & 1983 & 34 & 1 & - & - & 2 & 1 & - \\
Calvert & 1985 & 37 & - & 2 & - & - & 2 & - \\
Yerger & 1985 & 109 & - & - & 4 & - & 2 & - \\
Cabot & 1987 & 47 & - & - & - & - & 1 & - \\
Saillant & 1989 & 123 & 5 & - & 2 & - & - & - \\
& & & & & & & & \\
Total & & 1217 & 8 & 8 & 11 & 10 & 8 & 1 \\
\hline
\end{tabular}

Legend: N-Op: Number of operations performed; A- Hematoma; B-Disturbed wound healing; C-Synovial fistula; D- Limited range of elbow motion; E- Infection; G- Scar problems

\subsubsection{Prognostic variables of the outcome of operation}

Nollen (1981) and Saillant (1989) both found operative treatment less successful in tennis elbow caused by professional activities. They disagreed about the effect of age on the outcome of treatment. Nollen noticed that patients aged between 30 and 39 had poorer results than other patients, while Saillant found that 
patients over 50 were less successful. Operative treatment was also found to be less successful in patients who had failed to respond to any conservative treatment even temporarily (Nollen 1981) and in patients with a tennis elbow which had existed for more than two years (Saillant 1989).

\subsection{CONCLUSIONS}

Tennis elbow is a rather frequent pain syndrome at the lateral epicondyle, the pathogenesis of which still is not fully clear. The most widely supported view about its pathogenesis is that force overload causes (micro)-ruptures in the origin of the extensors of the forearm.

Few studies have been carried out to the incidence and prevalence rates of tennis elbow in the general population and no studies have been done to the numbers of people actually treated for a tennis elbow, or the type of treatment received. The incidence and prevalence of tennis elbow in general practice have not been studied in relation to the number of persons suffering from this condition in the general population.

Conservative treatment of tennis elbow has many modalities, the two commonest being corticosteroid injection and physiotherapy. Results of these treatments for tennis elbow have not been compared with each other.

Most studies of treatment results conducted by orthopaedic surgeons and rheumatologists have had a rather brief follow-up. Long-term treatment results in their selected patient populations seem to be disappointing.

Most operative procedures claim fairly good results on the basis of limited and retrospective studies. However, the severity and the extent of these operations vary greatly and no gold standard operation is available for comparison. 
CHAPTER

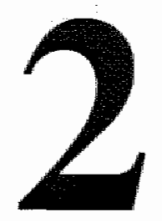

Anatomical aspects of tennis elbow 


\section{ANATOMICAL ASPECTS OF TENNIS ELBOW}

\section{1.}

\section{INTRODUCTION}

Few papers have concentrated on the anatomy of the lateral elbow region and most authors have used the rather limited description provided by the most current textbooks of anatomy. Over the last decade, however, some authors have produced new data about the anatomical features of the lateral elbow region (Heyse-Moore 1984, Briggs and Elliott 1985, Van Mameren and Drukker 1984). This has led to the concept of the Lateral Cubital Force Transmission System, which describes the way tensile forces generated by the muscle fibres of the forearm muscles are transmitted to the bony structures of the lateral epicondyle (Van Mameren and Drukker 1984). This concept offers a framework for the possible localisation of the macro- or micro-ruptures supposed to occur in tennis elbow. In this chapter we will review the traditional anatomical description of the anatomy of the elbow in relation to tennis elbow. This is followed by a description of the Lateral Cubital Force Transmission System.

\section{2.}

\section{ANATOMY OF THE LATERAL ELBOW REGION}

Joint

The ellbow joint is a compound synovial joint consisting of three articulations, the humero-ulnar, the humero-radial and the proximal radio-ulnar joint. The humero-ulnar and humeroradial joints form a uni-axial joint. The proximal radio-ulnar joint is a uni-axial pivot between the circumferences of the radial 
head and the osteofibrous ring made by the ulnar radial notch and the annular ligament. The annular ligament is a strong band which holds the radius against the radial notch of the ulna and forms four-fifths of the ring. Notch and ligament form a cupshaped socket that is wide proximally and narrow distally, thereby preventing distal migration of the radial head in relation to the ulna (Wadsworth 1982).

\section{Capsule}

The articular capsule is attached to the front of the medial epicondyle and humerus above the coronoid and radial fossa, and distally to the edge of the ulnar coronoid process and annular ligament. The capsule is continuous at its sides with the ulnar and radial collateral ligaments. Posteriorly, the capsule is attached to the humerus behind the capitulum to the edge of the olecranon and to the back of the medial epicondyle. Two collateral ligaments have been described (Williams and Warwick 1989) (Figure 2-1):

The ulnar collateral ligament (=medial cubital ligament) has a triangular form. It consists of three bands, continuous one with the other; an anterior, a posterior and an inferior band. The anterior part has its apex towards the medial epicondyle and its broad distal base towards the ulna. The posterior part also has a triangular form and is attached to the medial epicondyle and to the medial side of the olecranon. The inferior oblique ligament runs from the coronoid process of the ulna to the olecranon.

The radial collateral ligament (=lateral cubital ligament) is attached to the lateral epicondyle and to the annular ligament. Some of its fibres cross the annular ligament to the proximal end of the ulna. It is extensively blended with attachments of the supinator muscle and the extensor carpi radialis brevis.

The proximal border of the annular ligament blends with the cubital capsule. The annular ligament's external surface blends with the radial collateral ligament and is part of the attachment of the supinator muscle.

Vascular supply to capsule and synovial membrane comes mainly from the anastomosis of the collateral branches of the brachial artery and the recurrent branches of the radial and ulnar arteries. Antebrachial veins ascend with the radial and ulnar arteries, communicating with superficial veins, and emerge into the venae commitantes of the brachial artery at the elbow.

The anterior part of the capsule is innervated by a branch of the musculocutaneous nerve. The radial nerve supplies the posterior and anterolateral regions. The ulnar nerve supplies the ulnar collateral ligament and the median nerve gives off a small branch to the region of the medial epicondyle (Gardner 1948). 


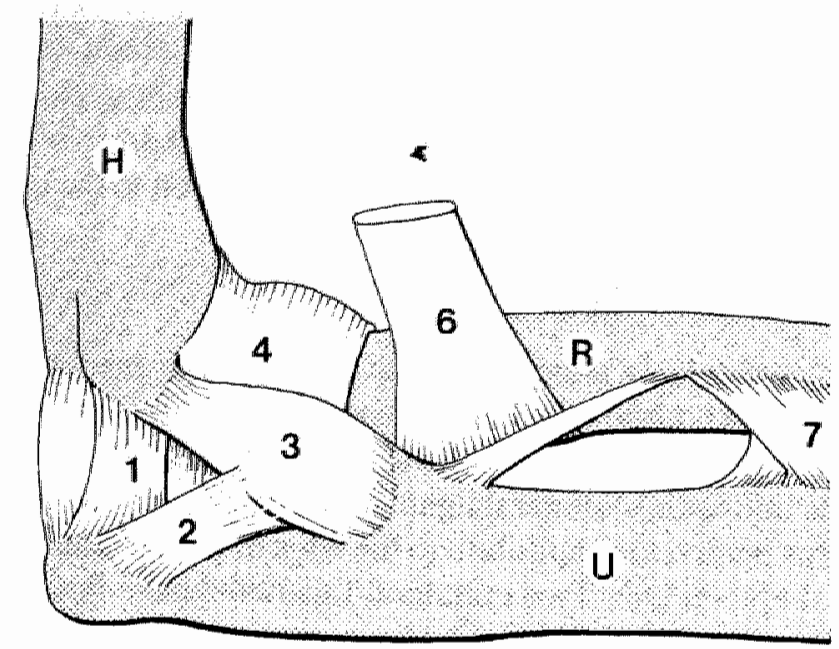

a

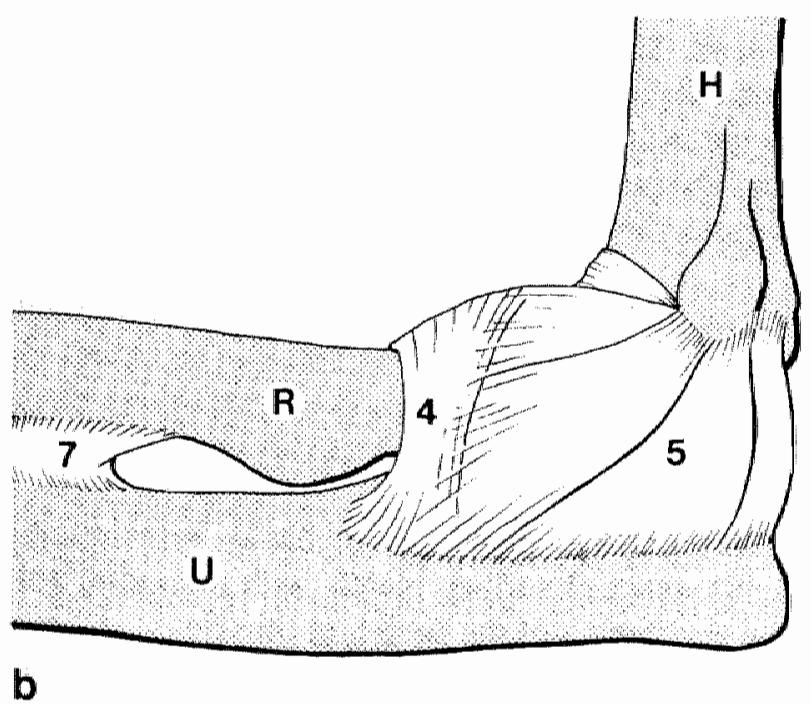

Figure 2-1.

Left elbow joint: Medial (a) and lateral (b) aspect.

Legend: humerus $(\mathrm{H})$; ulna $(\mathrm{U})$; radius $(\mathrm{R})$; posterior $(1)$, oblique (2) and anterior band of ulnar collateral ligament (3); annular ligament (4); radial collateral ligament (5); tendon of biceps (6); interosseous membrane (7). 
Table 2-1.

Muscles of the forearm

\author{
Anterior Antebrachial Muscles \\ Superficial group \\ pronator teres \\ nexor carpi radialis \\ palmaris longus \\ flexor carpi ulnaris \\ flexor digitorum superficialis \\ Deep group \\ flexor digitorum profundus \\ flexor pollicis longus \\ pronator quadratus
}

\title{
Posterior Antebrachial Muscles \\ Superficial group \\ brachioradialis \\ extensor carpi radialis longus \\ extensor carpi radialis brevis \\ extensor digitorum \\ extensor digiti minimi \\ extensor carpi ulnaris \\ Deep group \\ supinator \\ abductor pollicis longus \\ extensor pollicis brevis \\ extensor pollicis longus \\ extensor indicis
}

\section{Muscles}

The antebrachial musclles are divided morphologically into flexors and extensors of the wrist and fingers (anterior and posterior), but they often co-operate in more complex activities. The anterior and posterior antebrachial groups are divided into a superficial and a deep group (Table 2-1).

The anconeus muscle is considered to belong to the triceps region. All muscles of the posterior superficial group arise from the lateral epicondyle, except the brachioradialis and extensor carpi radialis longus.

Because of the rellation with the subject of this thesis the posterior muscles, which are located near the lateral epicondyle, will be discussed in further detail: 
- Brachioradialis muscle: The muscle arises from the proximal two-thirds of the lateral supracondylar ridge and anterior surface of the lateral intermuscular septum. The muscle forms a flat tendon attaching on the distal end of the radius, proximal to its styloid process. Nerve supply by the radial nerve (C5- C6- C7). - Extensor carpi radialis longus muscle (ECRL): The ECRL arises from the distal one-third of the lateral supracondylar ridge and partially from the common extensor tendon and is distally attached to the second metacarpal base. Nerve supply by the radial nerve (C6-C7).

- Extensor carpi radialis brevis muscle (ECRB): The ECRB is attached to the lateral epicondyle, the radial collateral ligament, to the antebrachial fascia that is aponeurotically reinforced and to adjacent intermuscular septa. Distally, it is attached to the dorsal proximal side of the third metacarpal. The posterior interosseus nerve innervates the ECRB (C7-C8).

- Extensor digitorum muscle $(E D)$ : The muscle arises from the lateral epicondyle by the common extensor tendon, from the adjacent intermuscular septa and the antebrachial fascia that is aponeurotically reinforced. Distally, it forms four extensor tendons of the fingers. The muscle is innervated by the posterior interosseous nerve ( $\mathrm{C} 7-\mathrm{C} 8$ ).

- Extensor digiti minimi muscle (EDM): The muscle is usually connected with the extensor digitorum, arising from the common extensor tendon and the adjacent intermuscular septa. It extends the fifth digit. As the extensor digitorum it is supplied by the posterior interosseous nerve (C7-C8).

- Extensor carpi ulnaris muscle (ECU): The ECU is attached to the lateral epicondyle by the common extensor tendon and to the middle two-fourths of the posterior ulnar border. Its tendon ends on the ulnar side of the base of the fifth metacarpal bone. The posterior interosseous nerve innervates the muscle (C7-C8). - Supinator muscle: The supinator surrounds the proximal third of the radius and has a superficial and deep leaf between which the posterior interosseous nerve passes the muscle, which is also innervated by this nerve (C5-C6). The muscle arises from the lateral epicondyle, radial collateral ligament, annular ligament and the supinator crest of the ulna. Distal attachment is to the lateral surface of the proximal third of the radius, reaching the distal attachment of pronator teres.

- Anconeus muscle: The anconeus is partially blended with the triceps. Its tendon is attached postero-distally to the lateral epicondyle and its muscle fibres are attached to the olecranon and the shaft of the ulna. It is innervated by the radial nerve (C7-C8) (Williams and Warwick 1989).

\section{Arcade of Frohse}

At the level of the humero-radial joint the radial nerve divides into its major branches, the posterior interosseous nerve and the superficial radial nerve. The posterior interosseous nerve passes between the two heads of the supinator 
muscle. The proximal edge of the supinator forms an arch for the posterior interosseous nerve, the arcade of Frohse (Spinner 1986). The arcade of Frohse may have a well developed fibrous edge at its intersection with the nerve (Werner 1979).

\section{Movements}

The ulna moving on the trochlea and the radial head on the humeral capitulum result in flexion and extension. This swing is, however, accompanied by slight conjunct rotation. The ulna internally rotates by approximately 5 degrees at the humero-ulnar articulation in early flexion and externally rotates by nearly 5 degrees during terminal flexion (Wadsworth 1982). Flexion is limited chiefly by soft parts, but in full flexion the tip of the ulnar coronoid enters the coronoid humeral fossa. Extension is limited by the tension in the capsule and the muscles anterior to the joint on the one hand, and the entry of the tip of the olecranon in the olecranon fossa of the humerus on the other.

The axis of rotation for pronation and supination is often represented as a line through the center of the radial head (proximal) and the ulnar attachment of the articular disc (distal). The axis of rotation, however, does not remain stationary and is far more complex than many authors have suggested. (Williams and Warwick 1989).

\section{Subtendinous space}

Goldie (1964) drew attention to the subtendinous space beneath the common extensor origin. The space is bounded proximally by the lateral epicondyle, anteriorly and laterally by the common extensor origin, posteriorly by the anconeus and medially by the joint capsule (Hughes 1950, Goldie 1964). Others have described a bursa in the same area (Osgood 1922, Stack 1949). Goldie (1964) found no signs of a primary bursa in the subtendinous space, which normally consists of areolar tissue. He claims it becomes filled with granulation tissue in patients with tennis elbow. Briggs and Elliot (1985), however, noticed a bursa between the aponeurosis and the underlying bone in 9 out of 139 cadaveric arms $(5 \%)$. These authors concluded that the presence of a bursa in the anatomical specimen may represent an acquired structure rather than an anatomical one.

\subsection{ACTION OF THE POSTERIOR ANTEBRACHIAL MUSCLE GROUP}

Almost all muscles have several functions, depending on the position of rotation of the forearm or the position of wrist, fingers or thumb. However, the following general outline can be given (the humerus being considered in a fixed position) (Williams and Warwick 1989): 
- Supination of the forearm: The supinator acts alone in slow, unopposed supination, but is joined by biceps brachii in fast or forceful supination.

- Extension of the wrist: The extensor carpi radialis brevis and longus, together with extensor carpi ulnaris, extends the wrist. The extensor carpi radialis longus is less active in pure extension than the brevis and is more active than the brevis in grasping. Both muscles work synergistically with the flexor carpi radialis for the abduction of the wrist. The extensor carpi ulnaris acts synergistically with the flexor carpi ulnaris, preventing abduction of the wrist when the pollex is extended.

- Extension of the digits: The extensor digitorum, together with the lumbricales, interossei and abductor digiti minimi, forms a complex digital extensor mechanism. The second and fifth fingers have an additional extensor muscle, the extensor indicis and extensor digiti minimi.

In tennis elbow, contraction of the wrist and finger extensors may induce pain. Several resisted movements of forearm, wrist and fingers have been described for the diagnosis (chapter 1, 1.2.2).

From anatomical data it may be concluded that:

1. Resisted dorsiflexion of the wrist may lead to activity in the extensor carpi radialis longus and brevis and the extensor carpi ulnaris.

2. Resisted dorsiflexion of individual fingers may provoke tension in different areas of the common extensor origin.

3. Resisted pronation and supination of the forearm usually activate more than just the rotators of the forearm, because synergistic activity arises in the extensors and flexors of the wrist.

4. Grasping (which is an activity of the flexors) leads to contraction of the extensors of the wrist, because the flexors of the fingers must be counterforced by the extensors of the wrist. This may explain the pain in tennis elbow during this activity.

\subsection{LATERAL CUBITAL FORCE TRANSMISSION SYSTEM}

Regular dissection techniques of the elbow separate muscles from the joint, capsule and bone. If the superficial antebrachial fascia is incised and muscle tissue is removed, then the coherence between capsular and intermuscular septa remains undisturbed. Thus, visualization of the relation between the regular 


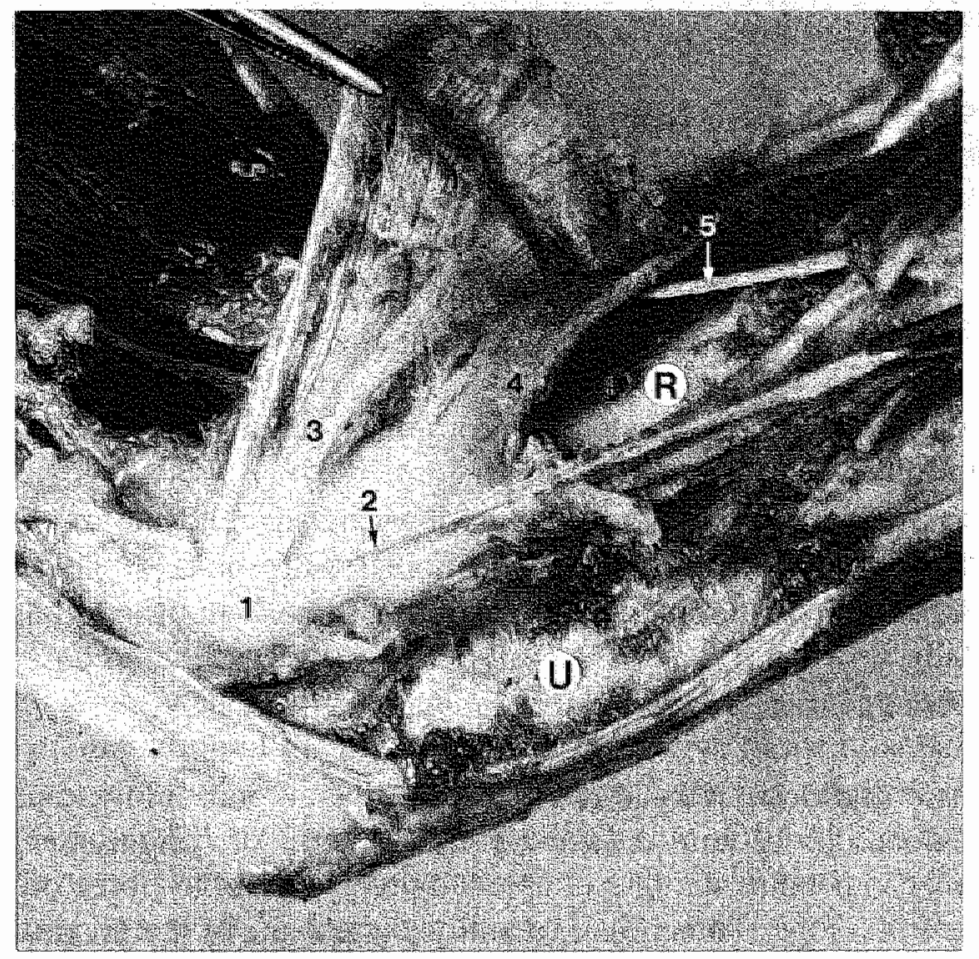

Figure 2-2.

Right cubital region in 50 degrees of flexion of the elbow and semipronation. The skin, subcutaneous tissue and the brachioradialis and extensor carpi radialis longus muscles has been removed. The muscle fascicull of the other muscles have been removed from their connective tissue sheaths. (From Mameren, van H, Lataster A, Rensema J, Drukker J : Acta Morphol Neerl-Scand 1985;23:247-58.)

Legend: ulna $(U)$; radius $(\mathbb{R})$; epicondyle (I); septum between the extensor carpi ulnaris muscle on one side and the extensor digitorum and digiti minimi muscle on the other side (2); septum between the extensor digitorum muscle on one side and the extensor carpi radialis brevis on the other side (3); supinator sheath (4); posterior interosseous nerve (5).

dense connective tissue of the muscle tissue and the elbow joint tissue can be achieved (Figure 2-2). This has led to the following findings (Van Mameren and Drukker 1984): 


\section{Extensor carpi radialis brevis muscle}

All muscle fibres of the extensor carpi radialis brevis attach to the septum between the extensor carpi radialis brevis and the extensor digitorum. No fibres are artached to the superficial fascia or directly to the lateral epicondyle.

\section{Extensor digitorum muscle}

The muscle fibres of the extensor digitorum are proximally surrounded by a sheath of regular dense connective tissue formed by

- the septum between the extensor digitorum muscle and the extensor carpi radialis brevis

- the septum between the extensor digitorum muscle and the extensor carpi ulnaris.

- the antebrachial fascia superficial to the muscle fascicles.

The extensor digitorum muscle consists of 4 separate musculotendinous units. The musculotendinous unit of the extensor of the middle finger attaches proximally directly distal to the lateral epicondyle. The muscle fibres of the other extensors of the fingers attach more distally to the walls of the sheath described above:

- The muscle fibres of the extensor of the second finger attach to the septum of the extensor digitorum muscle and the extensor carpi radialis brevis.

- The muscle fibres of the extensor of the fourth and fifth fingers attach to the septum between the extensor digitorum muscle and the extensor carpi ulnaris, and partially to the extensor digiti minimi.

\section{Extensor carpi ulnaris muscle}

Proximally, some muscle fibres attach to the lateral ulnar shaft, others to the overlying antebrachial fascia and some to a regular dense connective tissue aponeurosis deep to the muscle. This aponeurosis is not attached to the ulnar shaft. It is proximally continuous with the septum between the extensor digitorum and extensor carpi radialis brevis. 


\section{Supinator muscle}

The fibres of the supinator are surrounded by a tube of regular dense connective tissue. The muscle fascicles extend between the radius and the inner side of this tube. This tube extends proximally around the radial head. At this level, fibrocartilaginous tissue covers the inside of the tube, where it is in contact with the radial head. The connective tissue fibres of the supinator tube spiral and attach to the humerus around the humeral capitulum. This arrangement shows similarities to the rotator cuff in the shoulder.

This complex of connective tissue has also been studied using macroscopic cross-sections, CT and MRI scanning (Van Mameren et al. 1985). Based on these findings a reconstruction of the complex was generated with the aid of a computerized drawing program (Figure 2-3). At the level of the radial head the annular ligament was not identifiable as single structure. It appeared to be a proximal continuation of the regular dense connective tissue tube belonging to the supinator muscle. The collateral ligaments were not recognizable either. They were embedded in the regular connective tissue of the whole complex. The orientation of the fibres in the region was similar to the direction of the fibres of the collateral ligaments as described in many anatomical textbooks.

Removal of all muscle fibres of extensor carpi radialis brevis, extensor digitorum, extensor carpi ulnaris and supinator reveals a system of regular dense connective tissue. At the level of the radial head and proximal to the radial head the fibres of the connective tissue are strongly intertwined, all converging to the lateral epicondyle. These results are supported by the dissection studies of Heyse-Moore (1984), who found that the origins of the extensor carpi radialis brevis and the superficial part of the supinator were blended and quite inseparable. According to this author, both muscles originate from the lateral epicondyle, the capsule of the elbow and the annular ligament, and contraction of either of these muscles would exert an equal pull on these structures. Briggs and Elliot (1985) also found that the extensor carpi radialis brevis did not solely arise from the lateral epicondyle, but also from the capsule of the elbow joint and the lateral intermuscular septum.

The concept that the regular dense connective tissue structures in the lateral cubital region are actually continuations of the walls of the muscle compartments in the forearm was further studied by Peters (1988) and Van der Wal (1988). Peters (1988) studied in vitro the septum between extensor digitorum and extensor carpi radialis brevis in man and found that different parts of the proximal septum were stressed when different distal tendons of the extensor 

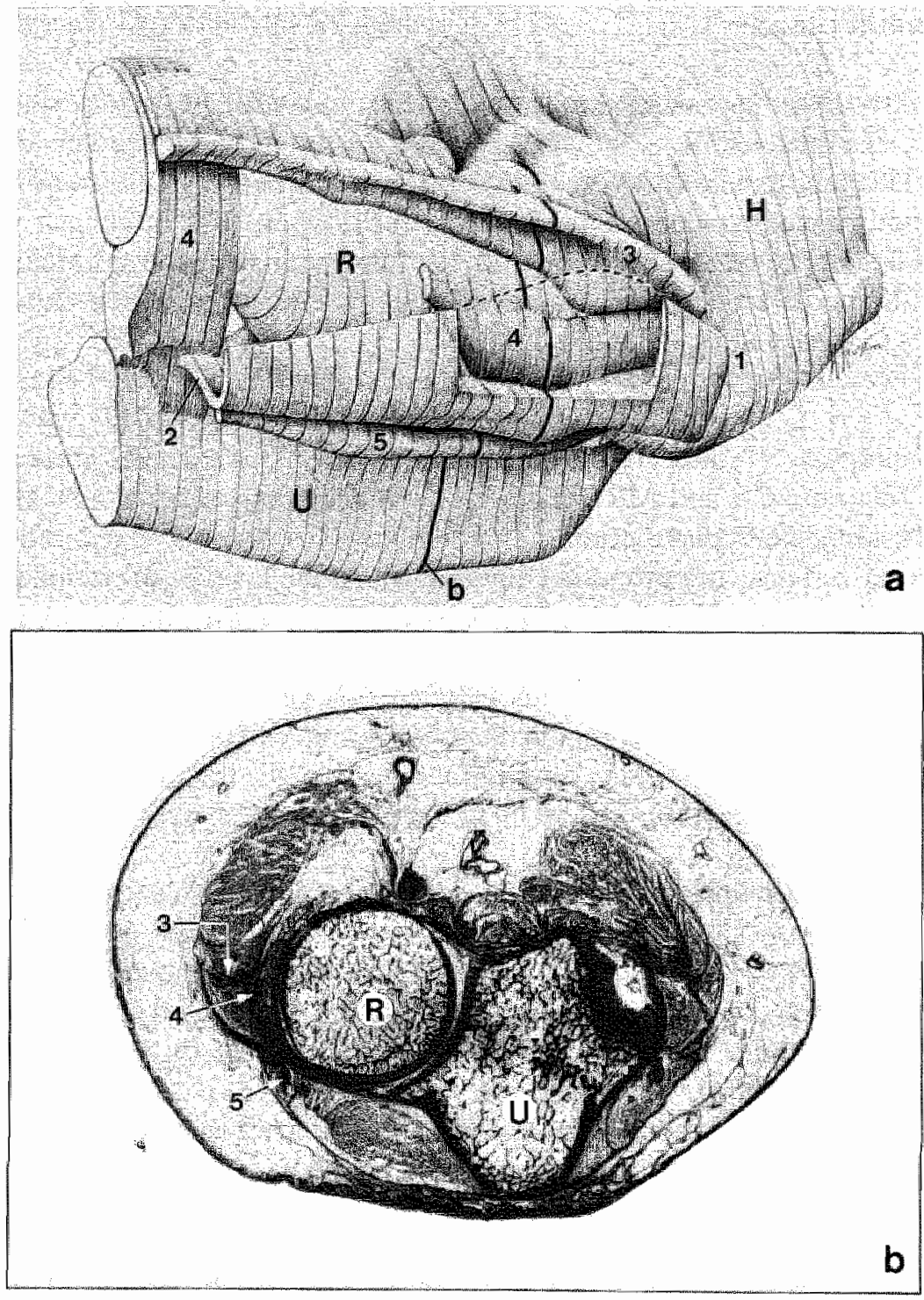

Figure $2 \cdot 3$.

A- A reconstruction of the intermuscular septa and collagenous fibres generated with the aid of a computerized drawing program. (From Mameren, wan H, Lataster LMA, Rensema $J$, Drukker J: Acta Morphol Neerl-Scand $1985 ; 23: 247-58$.) B-20 micrometers thick transverse section through the area indicated in Figure 2-3-A by means of a black line. (From Lataster LMA, van Mameren $H$, Terwindt E.: Anat Rec in press)

Legend: ulna $(U)$; radius $(\mathbb{R})$; epicondyle $(1)$; septum between the extensor carpi ulnaris muscle on one side and the extensor digitorum and digiti minimi muscle on the other side (2);septum between the extensor digitorum muscle on one side and the extensor carpi radialis brevis on the other side (3); supinator sheath (4); tendon of the anconeus muscle (5). 
digitorum were stressed. Van der Wal (1988) investigated the morphological substrate of proprioception in this region (in rats) and concluded that the spatial orientation was in accordance with the architecture of the regular dense connective tissue as described above.

\section{$2.5 \quad$ CONCLUSIONS}

The general conclusions from these anatomical studies are that most of the regular dense connective tissue at the lateral epicondyle is connected in series with the intermuscular septa and the sheaths of the extensor muscles and the supinator. The system is rather complex and the transmission of muscular forces at the lateral epicondyle may have different patterns, depending upon the muscles activated. If a rupture were to arise in the LCFTS, it might be expected that most pain is produced by the contraction of those muscles originating from that part of the LCFTS. 
CHAPTER

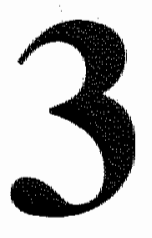

Epidemiology of tennis elbow

a study of the prevalence in the general population and the incidence and treatment in general practice 


\section{EPIDEMIOLOGY OF TENNIS ELBOW}

a study of the prevalence in the general population and the incidence and treatment in general practice

\subsection{INTRODUCTION}

It is predominantly those patients whose livelihood or continuation of sport or hobby depends on adequate functioning of the arms who will be treated for tennis elbow (Kurppa et al. 1979). Thus, the actual number of people suffering from this condition may be higher than the estimations based on figures provided by general practitioners and hospitals. Only epidemiological studies in the general population can clarify the size of this group. Studies of the incidence and prevalence rates of tennis elbow in the general population have been scarce and insufficient, with the exception of the study by Allander (1974) and the epidemiological study in Zoetermeer (EPOZ 1979). However, neither of the studies analyzed the number of people actually treated for a tennis elbow, or the type of treatment received. This led us to design an epidemiological study to analyze the prevalence of tennis elbow in the general population as well as the way it is treated.

In the Netherlands, treatment for tennis elbow is mostly done by general practitioners. Studies by Lamberts (1975) and Blanken (1981) revealed an incidence of 4 to 5 per 1000 patients per year in general practice. However, in order to link the information from epidemiological studies in the general population with those in general practice the studies leading to these data should be carried out simultaneously, so as to correct the influence of seasonal fluctuations in incidence of tennis elbow. For that reason we analyzed the incidence of tennis elbow in 19 general practices in the same period when we also conducted our 
Table 3-11.

Indications for hospital admission or radiographic referral

\begin{tabular}{|c|c|c|c|}
\hline & $\mathbf{N}$ & $\%$ & \\
\hline Uterus/vagina prolapse & 22 & 3 & \\
\hline Cysto/rectocele & 3 & 1 & \\
\hline Uterus myomatosus & 63 & 9 & \\
\hline Cataract & 71 & 10 & \\
\hline \multicolumn{4}{|l|}{ Inguinal hernia } \\
\hline left & 46 & 7 & \\
\hline right & 54 & 8 & \\
\hline bilateral & 10 & 1 & \\
\hline \multicolumn{4}{|l|}{ Femoral hernia } \\
\hline left & 2 & 0 & \\
\hline right & 1 & 0 & \\
\hline emorrhoids & 2 & 0 & \\
\hline \multicolumn{4}{|l|}{ Varices } \\
\hline left & 17 & 2 & \\
\hline right & 13 & 2 & \\
\hline bilateral & 23 & 3 & 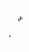 \\
\hline Coxarthrosis & 66 & 9 & \\
\hline Hallux valgus & 31 & 4 & \\
\hline Removal osteosynthetic material & 14 & 2 & \\
\hline \multicolumn{4}{|l|}{ Roentgenographic referral } \\
\hline ultrasonography gall bladder & 137 & 20 & \\
\hline abdominal ultrasonography & 83 & 12 & \\
\hline Miscellaneous & 50 & 7 & \\
\hline Total & 708 & 100 & \\
\hline
\end{tabular}

epidemiologic study in the general population. In addition, we studied the treatment of tennis elbow by the general practitioner, the treatment results and the referral pattern.

The first part of each section describes the epidemiological study in the general population. The study carried out in general practice is presented in the second part of each section of this chapter. 


\subsubsection{Study in the general population}

The prevalence of tennis elbow was investigated concurrently with an epidemiological study of gall stones and their relation with dyspepsia. Patients who were admitted to the Academic Hospital Maastricht or referred to this hospital for a radiographic abdominal examination were asked to participate in this dyspepsia study (Thijs et al. 1992). The study was performed by the Department of Epidemiology and Biostatistics of the University of Limburg.

Supplementary to the extensive questionnaire on the dyspepsia-study, information was gathered about the prevalence and treatment of tennis elbow. Our study only included those patients who were referred to the hospital for diseases without known association with tennis elbow. Table 3-1 lists the indications for admission or referral of the persons included in the study.

The patients were interviewed by one trained research assistant using a standardized questionnaire. Subjects were asked whether they felt any pain at the lateral elbow which increased at dorsiflexion of the wrist or fingers (this was demonstrated to the subject) at the time of the inquiry. If they responded affirmatively, the side of the complaints, the duration of complaints and the way they had been treated were recorded. All subjects were also asked if they had suffered such complaints in the past. If the answer was affirmative, data similar to the present complaints described above were recorded.

The diagnosis of tennis elbow was based on a combination of the following two criteria:

1- complaints of pain at the lateral side of the elbow.

2- provokable pain at the lateral epicondyle upon resisted dorsiflexion of the wrist.

These criteria were chosen according to the suggestions of Waris et al.(1979) who developed diagnostic criteria for the screening of neck and upper limb disorders. Waris et al. (1979) also included local tenderness of the lateral epicondyle at palpation in their diagnostic criteria, but we excluded this criterium from our study, since our inquiry was in most cases not performed by a physician. 
Table 3-2.

Differences in age between the subjects in the study and the general population

\begin{tabular}{|c|c|c|c|c|c|c|}
\hline \multirow[b]{2}{*}{ Age } & \multicolumn{3}{|c|}{ Males } & \multicolumn{3}{|c|}{ Females } \\
\hline & $\mathrm{N}$ & \% group & $\%$ population & $\mathbf{N}$ & \% group & \% population \\
\hline $20-39$ & 58 & 23 & 47 & 105 & 23 & 44 \\
\hline $40-59$ & 87 & 35 & 34 & 215 & 47 & 33 \\
\hline $60-80$ & 105 & 42 & 19 & 138 & 30 & 23 \\
\hline
\end{tabular}

250 males and 458 females were available for analysis (response rate: males $81 \%$, females $79 \%$ ). The mean age of the subjects was 51.8 years (range $20-80$ years). There were differences in age between the subjects in the study and the general population (Table 3-2). This table shows that subjects were older than the general population. Moreover, there were more women in our group as shown in table $3-3$, especially in the younger age category.

Table 3-3.

Differences in male-female ratio between the subjects in the study and the general population

Sex-ratio male - female

Age

Group studied

General population

20-39

$35.6-64.4$

$50.9-49.1$

$40-59$

$28.8-71.2$

$50.5-49.5$

$60-80$

$43.2-56.8$

$42.5-57.5$

From the age-specific prevalence figures we calculated the expected prevalence in the general population of our region (Zuidelijk Zuid-Limburg), for the age between 20 and 80 .

\subsubsection{Study in general practice}

Nineteen general practitioners were asked to participate in this part of the study. The mean number of patients of the practices was 2500 (range 1300-4000 patients). The total practice population consisted of about 47,500 persons. 
All patients presenting for the first time with tennis elbow during a one-year period were recorded on a precoded form, which included items about age, onset of symptoms, cause of symptoms, pain tolerance of the patient, severity and localization of the pain, pain provoked by resisted movements, treatment and result of treatment and character of the patient (fighter or flighter). The last description was solely based on the impression of the general practitioner.

Inclusion in the study was based on the following criteria.

1 complaints of pain at the lateral side of the elbow.

2 provokable pain at the lateral epicondyle with resisted dorsiflexion of the wrist.

3 local tenderness of the lateral epicondyle at palpation

The first two criteria were also used in the epidemiological study in general population.

The treatment was left to the judgement of the general practitioner and was registered in the study records. The preliminary results of treatment were described at the end of treatment by the general practitioner after a patient had been cured or when he or she was referred.

One year after the first consultation, the general practitioner again evaluated the treatment results of the patients. The evaluation was done by personal examination in $59 \%$ of the patients, and by telephone in $41 \%$ of the patients. The result of treatment could be assessed as 'good' or 'poor'. 'Good' was defined as cure or distinct improvement of the complaints, based on the patient's own judgement. 'Poor' was defined as no improvement, recurrence of symptoms after a symptomfree interval or referral to the out-patient clinic of the hospital.

At evaluation the severity of remaining complaints was described, as well as the total duration of the treatment.

\subsubsection{Statistics}

All data are given as numbers of persons or patients and percentages of the entire group. 95\%-confidence intervals for the expected prevalence in the general population were based on normal approximation. In the study in general practice the correlation between some pre-treatment variables and the outcome of treatment was evaluated using the $\mathrm{X}^{2}$-test. 


\subsubsection{General population}

\section{Present tennis elbow complaints}

Thirty-one of the 708 participants $(4.4 \%)$ to the study had tennis elbow complaints at the time of the interview. Twenty-four subjects $(77 \%)$ had right-sided complaints, 3 subjects left-sided complaints and 4 subjects had bilateral complaints. Tennis elbow was rather rare in persons younger than 40 or older than 70 (Table 3-4). In the group of subjects between 40 and 60 years of age, 7 to $9 \%$ had tennis elbow complaints.

\section{Table 3-4.}

Number (percentage of age group) of subjects with tennis elbow complaints at the time of the interview in relation to their age

\begin{tabular}{|c|c|c|c|c|c|c|}
\hline \multirow[b]{2}{*}{ Age } & \multicolumn{3}{|l|}{ Merl } & \multicolumn{3}{|l|}{ Women } \\
\hline & Tennis Elbow & $(\%)$ & Total & Tennis Elbow & $(\%)$ & Total \\
\hline $20-29$ & 0 & $(0)$ & 12 & 0 & $(0)$ & 30 \\
\hline $30-39$ & 0 & $(0)$ & 46 & 1 & ( 1$)$ & 75 \\
\hline $40-49$ & 1 & (3) & 39 & 14 & (11) & 123 \\
\hline $50-59$ & 2 & (4) & 48 & 8 & ( 9$)$ & 92 \\
\hline $60-69$ & 0 & (0) & 55 & 4 & (5) & 83 \\
\hline $70-79$ & 1 & (2) & 50 & 0 & $(0)$ & 55 \\
\hline Total & 4 & & 250 & 27 & & 458 \\
\hline
\end{tabular}

The mean period of complaints was 44 months (range 1-120 months) (Table $3-5)$. Eighteen of the 31 subjects $(58 \%)$ had been or were being treated for their tennis elbow. It was remarkable that as much as one third $(7 / 20)$ of those subjects who had had complaints for over a year did not seek medical advice (Table 3-5).

The treatment had been or was being performed by the general practitioner in 16 subjects. This treatment consisted of analgesics (3), local corticosteroid injections (9), advice to restrict activities or combinations of treatments (4). Physiotherapy had been prescribed to 8 persons. Four subjects had been referred to an orthopaedic surgeon- 3 of them had been operated on. 
"Wable 3.5 .

Relation between duration of complaints and numbers of patients treated $(\mathrm{N}=31)$

Duration of complaints (months)

\begin{tabular}{lllllll} 
Treated & $0-3$ & $4-6$ & $7-9$ & $10-12$ & $12-24$ & $>24$ \\
\hline No & 3 & 1 & 1 & 1 & 4 & 3 \\
Yes & 1 & 0 & 1 & 3 & 2 & 11 \\
\hline
\end{tabular}

Tennis elbow complaints in the past

Sixty-four subjects $(9 \%)$ reported complaints of tennis elbow from which they had recovered completely (Table 3-6). This table includes 6 of the 31 subjects with complaints at the time of the interview who had an earlier episode of complaints. Fifty of the 70 subjects $(71 \%)$ had been treated for tennis elbow; 38 $(53 \%)$ by the general practitioner. Nineteen subjects $(27 \%)$ had been operated on for tennis elbow. The mean length of time between the period of complaints and the date of the interview was 9.6 years (range $0.5-35$ years). The mean duration of the period of complaints was 11 months (range 1-108 months). This mean duration is rather short compared to the mean duration of complaints in the group with current elbow complaints ( 44 months). The pain was right-sided in 46 subjects $(65 \%)$, left-sided in 21 subjects ( $30 \%)$ and bilateral in 3 subjects. Prevalence of tennis elbow

\section{Table 3-6.}

Number of persons with tennis elbow complaints in the past in relation to their age

\begin{tabular}{|c|c|c|c|c|c|c|}
\hline \multirow[b]{2}{*}{ Age } & \multicolumn{3}{|l|}{ Men } & \multicolumn{3}{|l|}{ Women } \\
\hline & Tennis Elbow & $(\%)$ & Total & Tennis Elbow & $(\%)$ & Total \\
\hline $20-29$ & 0 & $(0)$ & 12 & 0 & $(0)$ & 30 \\
\hline $30-39$ & 0 & $(0)$ & 46 & 3 & (4) & 75 \\
\hline $40-49$ & 6 & $(15)$ & 39 & 15 & (12) & 123 \\
\hline $50-59$ & 11 & $(23)$ & 48 & 15 & (16) & 92 \\
\hline 60.69 & 6 & $(13)$ & 55 & 10 & $(12)$ & 83 \\
\hline $70-79$ & 2 & $(4)$ & 50 & 2 & (4) & 55 \\
\hline Total & 25 & & 250 & 45 & & 458 \\
\hline
\end{tabular}


Table 3-7 shows the results of the calculation of the prevalence of tennis elbow in the population in our region (Zuidelijk Zuid-Limburg). Thus, the expected point prevalence in the general population is $1 \%$ in men $(95 \%$-confidence interval $0-2.6 \%)$ and $4 \%$ in women $(2.6-5.8 \%)$.

\section{Table 3-7.}

Prevalence of tennis elbow in the general population aged between 20 and 80 . Values are $\%$ of entire population $(\mathrm{SE}=$ Standard error)

\begin{tabular}{lll}
\hline \multicolumn{3}{c}{ Prevalence } \\
\hline Population & 2.7 & $($ SE 0.52) \\
Men & 1.3 & (SE 0.66) \\
Women & 4.2 & $($ SE 0.79) \\
\hline
\end{tabular}

Allander (1974) found a remarkably high prevalence of tennis elbow in women between 40 and 45 years of age $(10 \%)$. For that reason we calculated the prevalence ratio (for the entire population) for women, using intervals of ten years (Table 3-8). These figures show that as many as 1 in 10 women aged 40 to 60 have tennis elbow complaints.

\section{Table 3-8.}

Point-prevalence of tennis elbow in women in relation to age. Values in \% of age group

\begin{tabular}{lc}
\hline Age & Women \\
\hline $20-29$ & 0 \\
$30-39$ & 1 \\
$40-49$ & 11 \\
$50-59$ & 9 \\
$60-69$ & 5 \\
$70-79$ & 0 \\
\hline
\end{tabular}




\section{Incidence of tennis elbow}

A total of 247 new patients presented with tennis elbow over a period of one year. The mean number of patients per participating general practitioner was 13 , with a considerable range; over a one year period one physician reported only one patient with a tennis elbow, while another general practitioner saw 48 patients with tennis elbow. The total number of new patients corresponds to an incidence of 5.2 patients per 1000 patients per practice per year.

The main complaint of all but one patient was pain. Subjective loss of strength was noticed by 87 patients. Numbers of males and females seen were similar (121 vs. 126). The complaints had started acutely in $28 \%$ of the patients. The mean symptomatic period before presentation to the general practitioner was 8 weeks (range 1-98 weeks). The mean age of the patients was 48 years (range 20-81 years) (Figure 3-1).

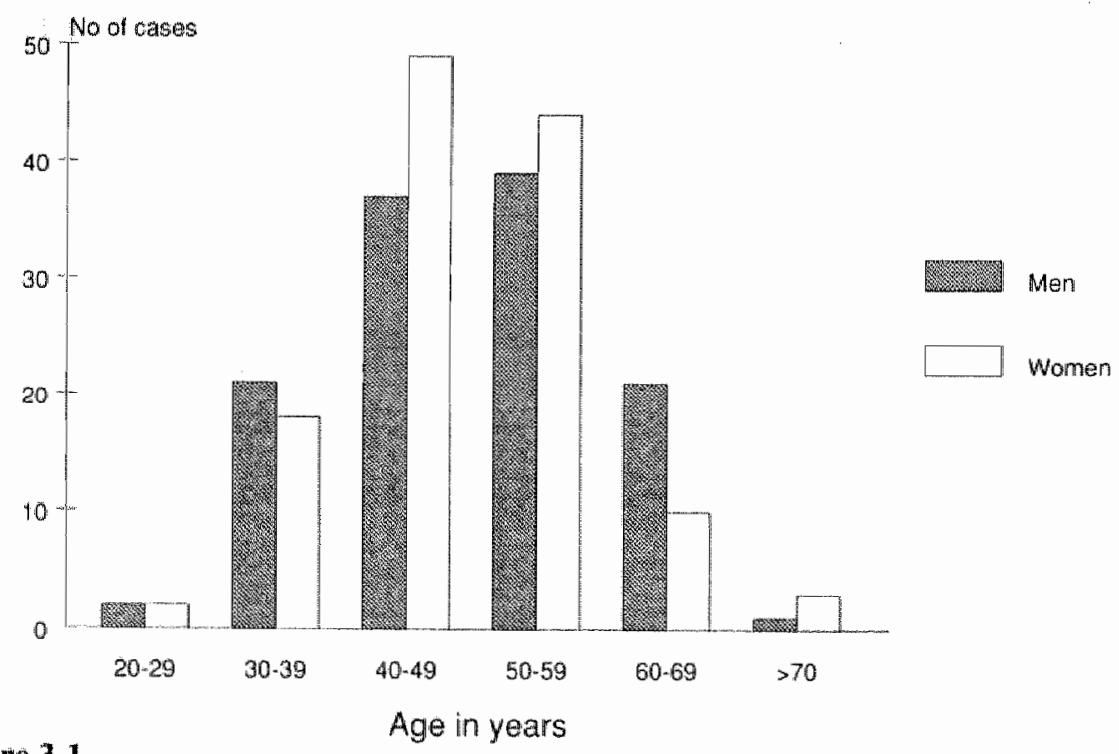

Figure 3-1.

Age distribution of the patients seen by the general practitioner. 
Nineteen per cent of patients consulted their general practitioner primarily for other reasons than the tennis elbow. Right-sided tennis elbow was more than twice as common as left-sided tennis elbow; $68 \%$ versus $29 \%$. Three per cent had a bilateral tennis elbow. Seventy-five per cent of left-dominant patients had complaints in their right elbow. Thus, the right arm was more often involved than the left arm, independent of the dominance of the $\operatorname{arm}(\mathrm{p}<0.001)$. Fifty-two of the 247 patients $(21 \%)$ who presented with a tennis elbow over a one-year period were recurrences after a painfree interval. The mean period between the two episodes was 19 months (range 2 months-9 years).

Ninety patients (36\%) thought there was an association between their professional activities and their symptoms. Twenty-six patients (11\%) noticed an association between sports activities and their tennis elbow. Twenty-five patients $(10 \%)$ presenting for the first time were on sick leave at that moment. The mean period during which they were unable to work was 11 weeks.

\section{Treatment}

The treatment of the tennis elbow was left to the judgement of the general practitioner consulted. Restriction of activities was advised in most patients $(68 \%)$ as the only therapy or in combination with other therapies. Seventy patients $(28 \%)$ received physiotherapy for a mean period of 6 weeks. The general practitioners gave an average of 2 corticosteroid injections to each of 93 patients (38\%) (range $\mathbb{1}-4$ injections). Bandages or splints were prescribed to 52 patients (21\%) and anti-inflammatory drugs or topical application of ointments was used in 86 patients ( $35 \%$ ). In most patients a combination of treatments was given, depending upon the reaction to prior treatment. Twenty-eight combinations of therapies were met. The treatment was completed after a mean period of 13 weeks (range 1-52 weeks).

231 patients $(93 \%$ ) could be followed up for one year. The remaining 16 patients had moved, could not be reached, had changed their general practitioner or had died. The results are shown in table 3-9. The results were classified as good or poor. 'Good' was defined as healed tennis elbow or distinct reduction in complaints. 'Poor" was defined as no improvement (unchanged or increased complaints), recurrence of symptoms or referral to the out-patient clinic of the hospital. 150 patients $(65 \%)$ had good treatment results and 81 patients $(35 \%)$ had poor results after treatment by the general practitioner. 
Table 3-9.

Results of treatment of tennis elbow in general practice

\begin{tabular}{lr}
\hline Healed tennis elbow & 108 \\
Reduced complants & 42 \\
Unchanged complaints & 18 \\
Increased complaints & 5 \\
Recurrence of symptoms & 19 \\
Referred & 39 \\
& \\
Lost to follow-up & 16 \\
\hline
\end{tabular}

Thirty-nine of the 81 patients with a poor result were referred to the orthopaedic out-patient clinic. Twenty-nine of the 111 patients younger than 50 were referred, as opposed to 10 of the 82 patients older than $50(\mathrm{p}<0.05)$. The general practitioner classified the patients on a 5-point scale for pain tolerance. Analysis of this pain tolerance versus the referral pattern showed that patients who were classified by their general practitioner as having a lower threshold for pain were more often referred than patients classified as having a high threshold $(\mathrm{p}<$ 0.001 ).

\section{Table 3-10.}

Predictors of a failure of treatment of tennis elbow in general practice

Prognostic negative factors \% poor result $\mathrm{p}$-value $\mathrm{x}^{2}$-test

Left-sided complaints

Right-sided complaints

'Flight' behavior

'Fight' behavior

Recurrence after prior treatment

No recurrence

Severe tennis elbow

Mild tennis elbow
37

23

55

35

47

33

47

27 $p<0.05$

$\mathrm{p}<0.01$

$\mathrm{p}<0.001$ 
At follow-up 7 patients (3\%) were still unable to work, $21(9 \%)$ patients had had to decrease their sports activities and 17 patients $(7 \%$ ) had changed their hobbies because of elbow complaints. Because of the high number of combinations of treatments it was impossible to analyze the results of each combination separately. The treatment results did not differ for males and females. The treatment results in patients presenting with a severe tennis elbow (pain in combination with loss of elbow motion and/or subjective loss of grip strength) were worse than those in patients with only lateral elbow pain $(p<0.01)$. Other factors associated with a poor result are summarized in table 3-10. If the period of treatment by the general practitioner exceeded three months, fewer than half of the patients achieved good treatment results $(p<0.001)$ (Table 3-11).

\section{Table 3-11.}

End results of treatment of tennis elbow in relation to duration of treatment

\begin{tabular}{lccc}
\hline & \multicolumn{2}{c}{ Results } & \\
\cline { 2 - 4 } Duration of treatment & Good & Poor & $(\%)$ \\
\hline$<12$ weeks & 104 & 32 & $(24)$ \\
$>12$ weeks & 46 & 49 & $(52)$ \\
Total & 150 & 81 & $(35)$ \\
Lost to follow-up: $16(6 \%)$ & & & \\
\hline
\end{tabular}

\section{4 .}

\section{DISCUSSION}

Ideal studies of the incidence and prevalence of a condition should be performed in a non-selected population suffering from that condition. In our study we used patients referred to our hospital as a substitute for the general population. None of the reasons for referral is known to be related to tennis elbow. However, it cannot be excluded that for example referral for abdominal complaints is related to a lower threshold of pain which may lead to a selection of persons with pain at the elbow.

The results of our epidemiological study in the general population show a prevalence for tennis elbow of $1 \%$ in men and $4 \%$ in women for the population aged between 20 and $80.28 \%$ of the population of our region is younger than 20 
or older than 80. At these ages tennis elbow is a rather rare condition (Chard and Hazleman 1989). Based on this assumption, the actual prevalence for the whole population might be $2 \%$. In Sweden, Allander (1974) found a prevalence for tennis elbow between 1 and $3 \%$ for both sexes. In women aged 42-46, however, he found a high prevalence rate, viz. $10 \%$. We found a comparable prevalence of $11.4 \%$ in the female age category of $40-49$. However, the prevalence rates for females up to the age 70 were also fairly high (Table 3-8).

The only other Dutch epidemiological study addressing the prevalence of tennis elbow was performed in Zoetermeer (EPOZ epidemiological survey). This study revealed prevalence of elbow complaints of $2 \%$ in men and $4 \%$ in women (EPOZ 1979). In the methods section we described the diagnostic criteria used in our epidemiological study. These criteria fit the diagnosis of tennis elbow, but will not exclude all other conditions, such as radial tunnel syndrome or radiohumeral osteoarthritis. In fact, what we have studied is the prevalence of lateral elbow pain. This means that the prevalence data given may slightly overestimate the prevalence of tennis elbow. However, similar criteria were used in other studies in the literature, which allows for comparison with ours.

Our study in general practice revealed an incidence of 5.2 per 1000 patients. Lamberts (1975) found an incidence of 5 per 1000 patients in 4 general practices in the Netherlands. The same number was found by Blanken (1981), reporting the incidence figures of 8 general practitioners. Van Lisdonk et al. (1990) reported an incidence of 6.6 for men and 6.4 for women per 1000 patients. In Great Britain, Hamilton (1986) noted an incidence rate of 4.2 per 1000 patients.

It has been shown that a considerable number of persons with lateral elbow complaints never seek medical attention. This is compatible with the notion that tennis elbow is often a self-limiting condition (Kamien 1990). About $60 \%$ of patients with current tennis elbow complaints and $70 \%$ of patients with previous episodes of these complaints had been treated, most of them by their general practitioner. In our study in general practice only new cases were registered in a one-year period (=incidence), so the study did not provide data about the actual number of patients suffering from the condition (=prevalence).

In general the following formula may be used:

$$
\text { Point prevalence }=\text { Incidence } * \text { Mean duration of complaints (in years) }
$$

The point prevalence we found in the general population was about $2 \%(0.02)$ and the mean duration of complaints was 11 months. Thus, the incidence in the general population will be about $2 \%(0.02=$ Incidence $\times 11 / 12)$. 
If half of all subjects with tennis elbow complaints were treated by the general practitioner the expected incidence in general practice would be $1 \%$. The actual incidence of tennis elbow in general practice was $0.5 \%$. The difference between this expected incidence $(1 \%)$ and the incidence ratio actually measured in general practice $(0.5 \%)$ may have several causes. The mean duration of complaints was different for those subjects with present tennis elbow complaints (mean 44 months) and those with tennis elbow complaints in the past (11 months). The relapsing character of the disorder may also have contributed to this difference. It is also possible that some persons with lateral elbow pain were not diagnosed as having a tennis elbow. Another source (Registratienet Huisartsenpraktijken RL) suggested, that the prevalence of tennis elbow in general practice in our region is $1.2 \%$. This figure suggests that there may have been some under-registration of tennis elbow in our study.

The epidemiological survey confirmed the findings of other authors that in most patients the mean period of complaints of tennis elbow is approximately one year (Cyriax 1982). There was, however, a wide range in the duration of the symptomatic period.

In general practice we found equal proportions of male and female tennis elbow patients, which is similar to the findings of other studies of tennis elbow (Hamilton 1986). Our own epidemiological study in the general population, as well as the study by Allander (1974), however, showed a higher prevalence of tennis elbow in females. Although the inclusion criteria were not fully identical for the two studies (local tenderness of the lateral epicondyle was not used as inclusion criterion in the study of the general population), this cannot explain the high percentage of tennis elbow complaints in females. It might mean that females do not present to their general practitioner with tennis elbow as often as men do. It might also indicate that the duration of complaints in females is longer than in males. Our data were inconclusive on that point.

Ten per cent of the patients with tennis elbow were unable to perform their job and were on sick leave. Because many women still do not have a paid employment, this figure represents an underestimation of the real incapacity provoked by tennis elbow. One fifth of the patients, however, presented with tennis elbow at consultation for another condition, which illustrates the benign nature of the condition in these patients.

No similar study in general practice is available to compare the results of our study. Forty-seven per cent of patients were cured and $18 \%$ improved. Nearly one sixth of the patients with a tennis elbow were referred to an out-patient clinic. These results of treatment must be interpreted in the light of the inherent 
limitations of studying a condition in 19 different general practices. The study was not intended to compare various treatment regimens, but was meant to analyze current treatment in general practice without influencing it, which is why we used a registration form. Differences in the accuracy of registration, the great variety of numbers of patients seen by the general practitioners, the various treatment regimens and the differences in assessment method (by telephone or by examination) may have influenced the outcome of the study. The methodological problems of scientific research in general practice are adequately described in the thesis by Jonquière (1986).

Patients who were referred to the specialist were more often classified by the general practitioner as having a lower threshold for pain than those who were not referred $(p<0.001)$. As was shown in table 3.10 , several other factors influence the outcome of treatment negatively. These data suggest that especially patients with severe complaints (subjective loss of grip strength, epicondylar redness or swelling, limitation of elbow motion) who do not respond to treatment within 12 weeks have a high risk of obtaining a poor treatment result. Further research in general practice should aim to limit the period of complaints especially for this group of patients.

\subsection{CONCLUSIONS}

From this study it may be concluded that:

- the prevalence of tennis elbow in the general population aged between 20 and 80 was 2.8 per cent.

- the prevalence for tennis elbow in females was higher than that in males and was 11 per cent in females between 40 and 50 years of age.

- approximately half of the patients with lateral elbow pain in the general population was actually being treated.

- in the general population the mean duration of tennis elbow complaints showed a wide range, with a mean duration of 11 months in the group with a cured tennis elbow.

- the incidence of tennis elbow in general practice was 5.2 per 1000 patients in one year, with an equal proportion of males and females. 
- in general practice a good result of treatment was obtained in two thirds of the patients.

- restriction of activities was the most commonly advised treatment in general practices, either as sole therapy or in combination with other therapies.

- seventeen per cent of the patients in general practice were referred to a specialist, among whom there were more patients with a lower threshold for pain, patients younger than fifty and patients with more severe complaints.

- if patients still had complaints after 3 months of treatment, more than half of them had a poor result at 1 year follow-up. 
CHAPTER

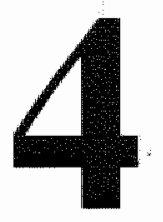

Conservative

treatment of tennis elbow

A prospective randomized trial comparing Cyriax' physiotherapy with corticosteroid injections 


\title{
CONSERVATIVE TREATMENT OF TENNIS ELBOW
}

\author{
A prospective randomized trial comparing Cyriax' \\ physiotherapy with corticosteroid injections
}

\subsection{INTRODUCTION}

Local corticosteroid injections and physiotherapy are among the most commonly applied treatment modalities of tennis elbow. The effectiveness of local corticosteroid injections has been established by several authors in randomized trials using saline or local anaesthetic as control (Bailey and Brock 1957, Day et al. 1978). After the initial reduction or disappearance of the complaints, however, several patients experience a recurrence of the lateral elbow pain (Freeland and Gribble 1954, Quin and Binks 1954, Murley 1954, Young et al. 1954). This recurrence rate has been reported to vary from 10 to 70 per cent over a period of six months (Clarke and Woodland 1975, Nevelös 1980). With the exception of the study by Binder et al. (1985) none have found physiotherapy to be more effective than placebo physiotherapy in the treatment of tennis elbow (Devereaux et al. 1985, Lundeberg et al. 1987, Lundeberg et al. 1988, Chard and Hazleman 1988). Cyriax (1936, 1982), however, claimed a high percentage of success in the treatment of tennis elbow using local friction in combination with Mills' manipulations. This claim has never been adequately evaluated, although Cyriax' method has found wide application in the Netherlands. No study has compared the effectiveness of local corticosteroid injections with physiotherapy in the treatment of tennis elbow. 
Therefore, we performed a study of these two common treatment modalities. In this chapter we describe the results of this study, which was set up as a clinical trial comparing the outcome and side effects in patients with tennis elbow randomized to treatment with local corticosteroid injections or to the physiotherapeutic method described by Cyriax. Severity of pain, grip strength and pain provoked by dorsiflexion of the wrist were chosen as principal outcome measures. Other outcome measures were tenderness at the lateral epicondyle and subjective loss of grip strength. Because recurrences of treatment can still be observed six months after treatment, we opted for a follow-up period of twelve months after the start of the treatment.

\subsection{PATIENTS AND METHODS}

\subsubsection{Selection of patients and randomization}

All patients with a tennis elbow referred to the departments of orthopaedics and general surgery of the Academic Hospital Maastricht were included in this study over a one year period.

The criteria for entry into the trial were:

1 pain at the lateral side of the elbow;

2 tenderness over the forearm extensor origin;

3 pain at the lateral epicondyle during resisted dorsiflexion of the wrist with the elbow in full extension.

One or more of the following factors led to exclusion from the study:

1 previous operation at the lateral side of the elbow;

2 arthritis or allied conditions;

3 neurological disorders of the painful extremity;

4 more than three local corticosteroid injections within a period of six months before consultation;

5 same elbow treated before with Cyriax' methods.

If patients had bilateral tennis elbow complaints, only the most painful arm was included in this trial.

All patients were seen at the 'elbow clinic' by an orthopaedic surgeon. If patients fulfilled all criteria for inclusion and there were no reasons for exclusion, patients were randomized using sealed numbered envelopes (Department of Medical Statistics, State University Limburg, A.Kester Ph.D.). A simple randomization method was used, without strata or blocks. The envelope contained the treatment to which the patient was to be allocated. 
It was considered unethical to continue the randomized treatment if treatment was entirely unsuccessful. At the follow-up after six weeks it was decided to continue or to alter the treatment in cases with persisting symptoms. This decision was not based on randomization. If patients had recovered and only slight elbow symptoms persisted, this was considered to be the end-point of treatment. The next scheduled evaluation for these patients was fifty-two weeks after the start of the treatment. However, patients were instructed to return to the out-patient clinic if they experienced moderate or severe symptoms. All patients who returned to the out-patient clinic after discharge were considered to have experienced a recurrence of symptoms. The investigator both provided the treatment and evaluated the results. He was, however, blinded for prior evaluations.

\subsubsection{Methods of treatment}

Two treatments were compared:

\section{1-Local corticosteroid injections}

The injection solution was triamcinolone acetate suspension $1 \%$, diluted with 1 cc lidocaine $1 \%$. This solution was injected into the tendinous origin of the extensor digitorum and extensor carpi radialis brevis. Starting from the point of maximal tenderness the triamcinolone solution was injected in different directions, in order to obtain optimal distribution. The patients were seen two and four weeks after the start of the treatment. A second or third injection was given if the patient had persisting complaints. The patients were advised not to use the affected arm for any activity which provoked pain during the six week treatment period.

\section{2-Physiotherapy according to Cyriax}

The physiotherapeutic treatment described by Cyriax was carried out at the physiotherapy department of the Academic Hospital Maastricht. Three experienced physiotherapists, who had attended a special course in 'Orthopaedic Medicine" (Cyriax), performed the treatments, which consisted of deep transverse friction over the extensor origin and Mills' manipulations. A total number of 12 treatments was given in the course of four weeks, followed by another two weeks of restriction of all painful activities. 


\subsubsection{Evaluation}

\section{Assessment of pretreatment variables}

Medical History:

Several variables such as severity and occurrence of pain and subjective loss of grip strength, were included. Patients were asked to grade these parameters by choosing from a set of 3 or 4 items (Table 4-1). Patients were asked whether the elbow pain radiated to their upper arm or forearm and whether they had shoulder or neck complaints. Other variables, such as dominance of the arm, onset of symptoms, cause of the complaints, profession and period of sick leave were also registered (Table 4-1).

\section{Table 4-1.}

Variables registered at intake of patients with tennis elbow

Severity of pain

Slight
Moderate

Severe

Occurrence of pain

Seldom

Regularly

Often/continuously

Radiation of pain

Upper arm

Forearm

Maximal pain

At rest

At activity

Localization

Very localized

More diffuse

Pain at night

Subjective loss of grip strength

Absent

Slight

Moderate

Severe
Cervical complaints

Shoulder pain

Onset of symptoms

Acute

Slowly progressive

Cause

Profession

Hobby

Sports

Side

Left

Right

Left and right

Dominant hand

Left

Right

Left and right

Sex

Male

Female

Age

Number of injections

Number of physiotherapy treatments 
Inspection: local swelling, depigmentation or atrophy of the skin were noted.

Range of motion: flexion and extension of the elbow, pronation and supination of the forearm were measured with a goniometer.

Pain provoked by resisted motion: The severity of pain around the lateral epicondyle provoked by contraction of various muscles during resisted movements were described by the patient as 'absent', "slight" or 'severe'.

- Elbow

Resisted contraction of the flexors and extensors of the elbow was carried out in full flexion and extension. During resisted pronation and supination the hand was in mid-position, with 90 degrees of flexion of the elbow. In both tests resistance was given by holding the forearm.

- Wrist

Resisted dorsal flexion, palmar flexion, ulnar and radial deviation were performed with an extended elbow and the forearm in 80 degrees of pronation.

\section{- Finger}

The same position of elbow and forearm was used for resisted extension of the extensor of the middle finger.

Palpation: The severity of tenderness was classified by the patient as absentslight-moderate-severe and the localization of the point of maximal tenderness was identified as described by Cyriax. Five locations of pain were distinguished (Figure 4-1):

\section{Type 1: (Supracondylar variety)}

Tenderness at the supracondylar origin of the extensor carpi radialis longus

Type 2: (Tenoperiosteal variety)

Tenderness at the anterior aspect of the lateral humeral epicondyle, and at the origin of the common extensor tendon to the bone

Type 3: (Tendinous variety)

Tenderness in the body of the common extensor tendon, 5 to $10 \mathrm{~mm}$ below the epicondyle

Type 4: (Muscular variety)

Tenderness at the proximal part of the muscle belly, at the level of the neck of the radius

Type 5: (not described by Cyriax)

Tendemess dorsolateral of the tip of the lateral epicondyle 


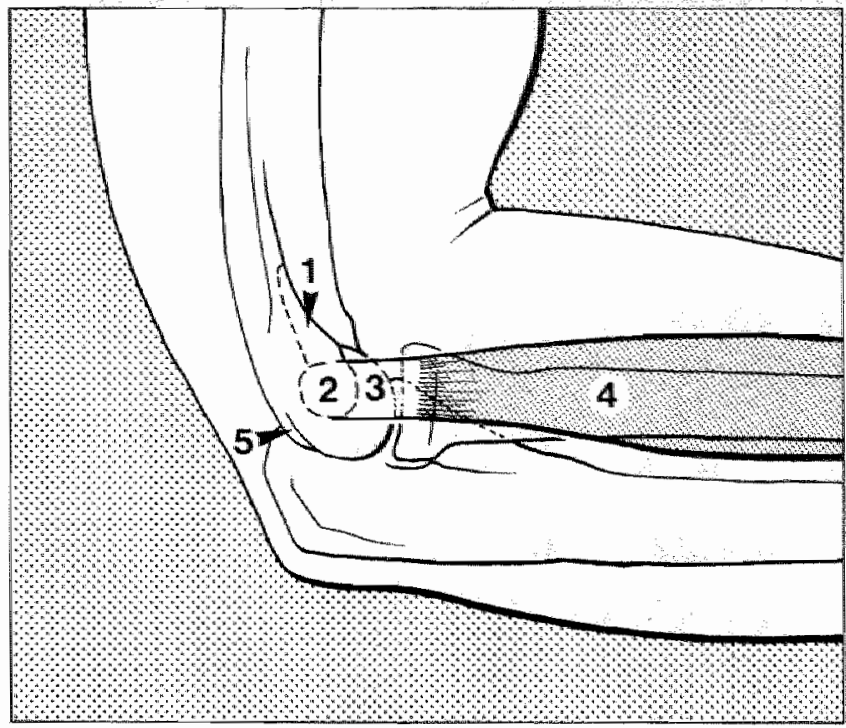

\section{Figure 4-1.}

The localization of the point of maximal tenderness. Five localizations were recognized.

The active ranges of motion of the cervical spine and the shoulder were examined and a limited neurological examination (including reflexes and sensibility) was performed.

\section{Grip strength}

Grip strength was measured using a Jamar ${ }^{(B)}$ digital hand dynamometer. The patient was unable to see the display during measurements. The grip strength of both hands was measured in kilograms. Measurements were performed with the elbow extended and in 90 degrees of flexion and the forearm in mid-pronation. For each position the average of two measurements was used (Figure 4-2).

During the study, the grip strength meter was damaged. The type of hand dynamometer used originally had been taken out of production and it could therefore not be replaced. It was substituted by a different model, with a digital display. Fortunately, a meter identical to the first one could be borrowed temporarily from another department. For a while, measurements were done using both meters in order to analyze differences in scaling between the two types of meters. We plotted the $\log$ (new/old) values against $\log ($ new/old) for the available "double" measurements. The correlation was low, from which we 


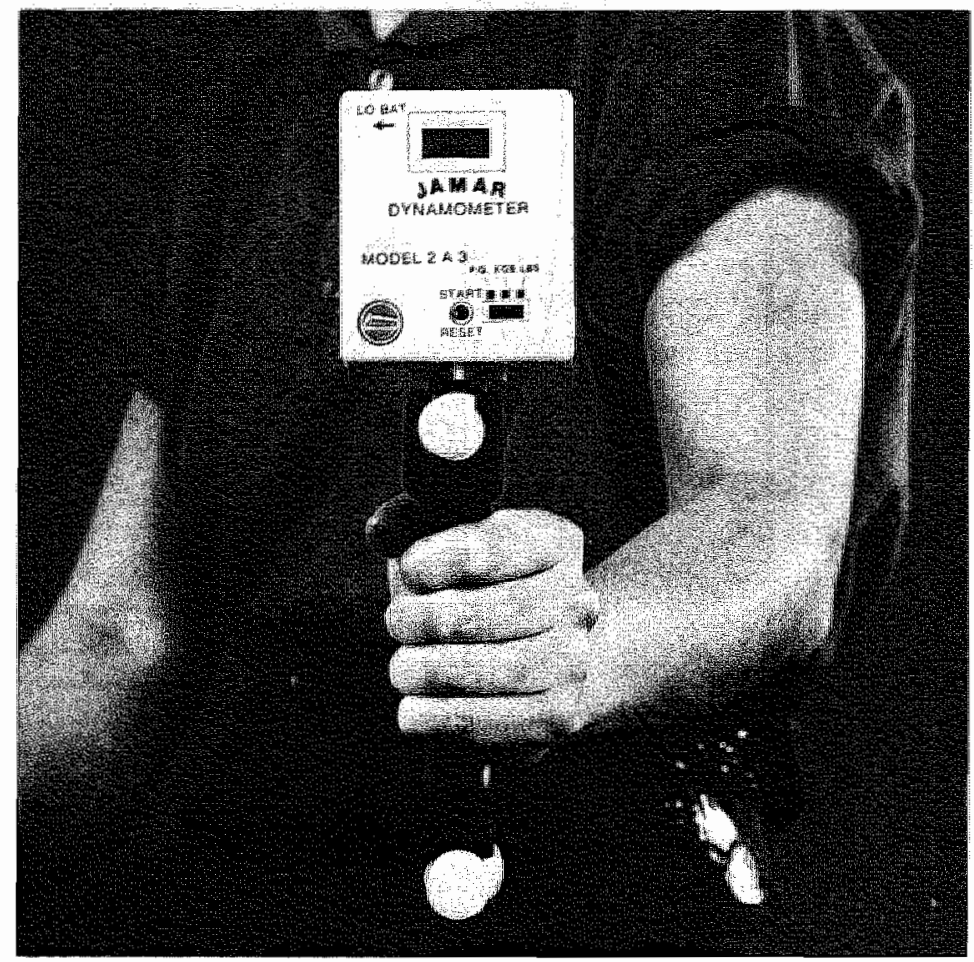

Figure 4-2.

The Jamar handdynamometer recording grip strength. The patient is not able to see the digital display.

concluded that the new/old ratio was apparently independent of the value itself. The mean of ${ }^{10} \log ($ new/old) was 0.198 (SD, 0.114), so the new meter's readings were approximately 1.58 times those of the old one. Subsequently, the measurements from the old meter were transformed to "new" through multiplication by 1.58. These values were analyzed.

\section{Radiographic examination}

$\mathrm{AP}$ and lateral radiographs were made of the affected elbows of all patients.

\section{Laboratory tests}

The sedimentation rate (Westgren) was determined in all patients.

\section{EMG examination}

Electromyographic and/or nerve conduction studies were performed only if neurological disorders were suspected. 


\title{
Assessment of results of treatment
}

The results of treatment were evaluated after six weeks and fifty-two weeks. The same pre-coded form for assessment of the results was used every time (Table 4-2). The presentation of the results of treatment below follows the sequence of variables in table 4-2.

Table 4-2.

Parameters used to evaluate treatment results of tennis elbow

\section{I- Anamnesis}

A-Severity of pain

B-Occurrence of pain

C-Subjective loss of grip strength

D-Return to work

\section{II- Physical Examination \\ A-Provocation tests (resisted motion) \\ B-Local tenderness \\ C-Localization of the point of maximal tenderness \\ D-Grip strength (hand dynamometer)}

\section{III-Conclusions}

A-Patient's assessment

B-Overall result rating

\begin{abstract}
All patients had at least two assessments, with the same intervals between start of treatment and assessment:
\end{abstract}

1- Evaluation after six weeks of treatment.

The treatment determined by randomization was not changed during the first six weeks. If this treatment turned out to be unsuccessful at the follow-up after six weeks, it was discontinued and a different conservative treatment was chosen. Conservative treatment was considered unsuccessful if the patient still had moderate or severe complaints and was unsatisfied with the outcome of treatment. Operative treatment was considered in patients with persisting complaints ( $>6$ months) and a long history of conservative treatment ( $>3$ months) by the general practitioner. The decision to operate, however, also depended upon the individual patient's functional requirements. 
2. Evaluation 52 weeks after start of treatment.

Patients were evaluated fifty-two weeks after the start of the treatment. The results were evaluated on the basis of the intention to treat principle.

The assessment made by the patient consisted of the following items:

- Satisfied

- Moderately satisfied

- Dissatisfied

For the overall ratings of the results of treatment made by the examining physician the following criteria were used:

$\begin{array}{ll}\text { Excellent: } & \text { - Complete relief of pain } \\ \text { Good: } & \text { - Occasional slight pain after strenuous activities } \\ & \text { - No pain provoked by resisted dorsiflexion of the wrist } \\ \text { Moderate: } & \text { - Improved; essentially no pain at might and slight pain at rest; } \\ & \text { discomfort after strenuous activities on a more tolerable level } \\ & \text { - Slight pain provoked by resisted dorsiflexion of the wrist } \\ \text { - No improvement } & \text { - Moderate or severe pain provoked by resisted dorsiflexion of the } \\ & \text { wrist }\end{array}$

\subsubsection{Statistics}

All data are given as numbers of patients, with the exception of the grip strength, which is reported as means $\pm S D$. Differences between the two treatment groups at 0 weeks, six weeks and fifty-two weeks were tested with the chi-square test for linear trend (Cochran 1954). P-values for the tests are given. Differences in the increase of grip strength were evaluated using two-sample T-tests.

Multiple regression was used to obtain a more accurate evaluation of the therapy results with correction for possible imbalances in the randomization, and to identify other predictors for the outcome at six weeks.

Grip strength (both in full extension and with the elbow in 90 degrees of flexion), overall result rating of the elbow, patient's assessment, severity of pain, subjective loss of strength and local tenderness at six weeks were used as outcome parameters. Of the pretreatment variables, randomized therapy (injections versus Cyriax), dominance of the elbow, grip strength with an extended elbow, severity of pain, cervical complaints, subjective loss of grip strength, local 
tendemess, and pain at resisted extension of the wrist and the middle finger were chosen as predictors at week 0 for the outcomes of treatment. For grip strength, multiple linear regression was used on the log-transformed values in order to improve compliance with the conditions for the linear regression model. For the other outcome variables, multiple logistic regression was used. In each analysis, the model was subsequently reduced by means of a step by step omission of non-significant predictors, without provoking a substantial shift in the coefficient of the therapy indicator. Further details of the analysis method are given in the appendix to this chapter.

\subsection{RESULTS}

\subsubsection{0 weeks: pretreatment variables}

\subsubsection{Anamnesis}

106 patients were included in the study: 59 men and 47 women, with a mean age of 43 years (standard deviation 9 years). The characteristics of the patients are listed in table 4-3.

No significant differences between the two treatment groups were found. Most patients considered a combination of factors to be responsible for the onset of their tennis elbow complaints (Table 4-3), professional activities being mentioned by half of the patients. Most patients (48) who considered their tennis elbow to be caused by professional activities said they had a heavy manual occupation. 16 patients described their job as light and 42 patients said they performed moderately heavy work. 36 patients were on sick leave (mean period 7 weeks). The other patients were still working or did not have a job or were receiving a disability pension.

92 of the 106 patients had been treated by their general practitioner. Medication had been prescribed for 34 of them, mostly NSAID's. The mean duration of the complaints was 33 weeks. An average of one corticosteroid injection (maximum three) had been given to the patients. The average number of physiotherapeutic treatments (no Cyriax' treatments) given was 8 per patient. Thus, most patients had been treated 12 times, which was the usual number of treatments prescribed in one series. 
Table 4-3.

Pretreatment clvaracteristics of the patients according to treatment group. Values are numbers (\%)

\begin{tabular}{|c|c|c|c|c|c|}
\hline \multirow{2}{*}{ Characteristics } & \multirow{2}{*}{ 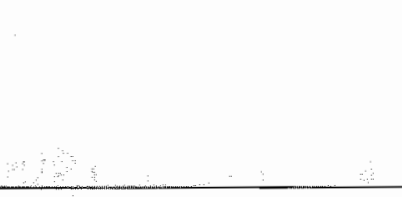 } & \multicolumn{4}{|c|}{ Treatment group } \\
\hline & & \multicolumn{2}{|c|}{$\begin{array}{l}\text { Physiotherapy } \\
(\mathrm{N}=53)\end{array}$} & \multicolumn{2}{|c|}{$\begin{array}{l}\text { Injections } \\
(\mathrm{N}=53)\end{array}$} \\
\hline \multirow[t]{3}{*}{ Severity of pain: } & Slight & 1 & $(02)$ & 2 & (04) \\
\hline & Moderate & 32 & $(60)$ & 30 & $(56)$ \\
\hline & Severe & 20 & $(38)$ & 21 & $(40)$ \\
\hline \multirow[t]{3}{*}{ Occurrence of pain: } & Seldom & 5 & $(09)$ & 4 & (08) \\
\hline & Regularly & 25 & $(47)$ & 22 & (41) \\
\hline & Often/continuously & 23 & $(44)$ & 27 & $(51)$ \\
\hline \multicolumn{2}{|c|}{ Radiation pain upper arm } & 24 & $(45)$ & 17 & (32) \\
\hline \multicolumn{2}{|c|}{ Radiation pain forearm } & 32 & $(60)$ & 32 & $(60)$ \\
\hline \multirow[t]{2}{*}{ Maximal pain: } & At rest & 11 & $(21)$ & 3 & (06) \\
\hline & At activity & 42 & $(79)$ & 50 & (94) \\
\hline \multirow[t]{2}{*}{ Localization: } & Very localized & 36 & (68) & 35 & (66) \\
\hline & More diffusely & 17 & $(32)$ & 18 & (34) \\
\hline Pain at night & & 33 & $(62)$ & 26 & (49) \\
\hline \multicolumn{6}{|c|}{ Subjective loss of grip strength: } \\
\hline & Absent & 10 & (19) & 6 & (11) \\
\hline & Slight & 11 & (21) & 12 & (23) \\
\hline & Moderate & 25 & $(47)$ & 28 & (53) \\
\hline & Severe & 7 & (13) & 7 & (13) \\
\hline \multicolumn{2}{|c|}{ Cervical complaints } & 15 & $(28)$ & 9 & (17) \\
\hline \multicolumn{2}{|l|}{ Shoulder pain } & 15 & $(28)$ & 8 & (15) \\
\hline \multicolumn{2}{|c|}{ Onset of symptoms: Acute } & 12 & (23) & 13 & (25) \\
\hline & Slowly progressive & 41 & $(77)$ & 40 & $(75)$ \\
\hline \multicolumn{2}{|l|}{ Cause profession } & 27 & $(51)$ & 26 & (49) \\
\hline \multicolumn{2}{|l|}{ Cause hobby } & 15 & $(28)$ & 13 & (25) \\
\hline \multicolumn{2}{|l|}{ Cause sports } & 5 & $(09)$ & 9 & (17) \\
\hline \multicolumn{2}{|l|}{ Cause unknown } & 14 & $(26)$ & 13 & (25) \\
\hline \multirow[t]{3}{*}{ Side: } & Left & 16 & $(30)$ & 14 & (26) \\
\hline & Right & 34 & (64) & 38 & (72) \\
\hline & Left and right & 3 & $(06)$ & 1 & (02) \\
\hline \multirow[t]{3}{*}{ Donuinant hand: } & Left & 3 & $(06)$ & 6 & (11) \\
\hline & Right & 48 & $(91)$ & 43 & (81) \\
\hline & Left and right & 2 & $(03)$ & 4 & (08) \\
\hline \multirow[t]{2}{*}{$\operatorname{Sex}^{\prime \prime}$} & Male & 28 & $(53)$ & 31 & (59) \\
\hline & Fennale & 25 & $(47)$ & 22 & $(41)$ \\
\hline \multirow{3}{*}{\multicolumn{2}{|c|}{$\begin{array}{l}\text { Mean (SE) number of corticosteroid injections } \\
\text { Mean (SE) number of physiotherapy treatments } \\
\text { Mean (SE) age in years }\end{array}$}} & 0.9 & $(1.1)$ & 0.7 & (1.1) \\
\hline & & 8.3 & $(11)$ & 6.7 & (10) \\
\hline & & 43.0 & $(8.5)$ & 42.6 & (9.9) \\
\hline
\end{tabular}




\subsubsection{Physical examination}

\section{Inspection}

Four patients showed a depigmentation of the skin at the injection site of the corticosteroid preparation given by the general practitioner. Some swelling around the lateral epicondyle was noticed in 11 patients.

\section{Range of motion}

The extension was limited in 7 patients. The extension limitation was induced by pain at passive or active extension.

\section{Provocation tests}

Table 4-4 lists the severity of pain provoked by resisted movements of elbow, wrist or hand.

Pain at resisted extension of the wrist was used as an inclusion criterion and therefore caused pain in all of our patients. Resisted extension of the middle finger was painful in 88 of the 106 patients.

\section{Table 4-4.}

Severity of pain provoked by resisted movements of elbow, wrist or hand in patients with tennis elbow $(\mathrm{N}=106)$

\begin{tabular}{|c|c|c|c|c|c|c|c|}
\hline \multirow[t]{2}{*}{ Pain } & & \multicolumn{2}{|l|}{ Absent } & \multicolumn{2}{|l|}{ Slight } & \multicolumn{2}{|l|}{ Severe } \\
\hline & & Cyriax & Injection & Cyriax & Injection & Cyriax & Injection \\
\hline \multirow[t]{4}{*}{ Elbow } & supination & 28 & 28 & 22 & $2 \|$ & 3 & 4 \\
\hline & pronation & 32 & 36 & 19 & 15 & 2 & 2 \\
\hline & extension & 47 & 46 & 6 & 6 & 0 & 1 \\
\hline & flexion & 47 & 46 & 6 & 6 & 0 & 1 \\
\hline \multirow[t]{4}{*}{ Wrist } & extension & 0 & 0 & 9 & 7 & 44 & 46 \\
\hline & palmar flexion & 50 & 50 & 3 & 3 & 0 & 0 \\
\hline & ulnar deviation & 37 & 31 & 15 & 21 & 1 & 1 \\
\hline & radial deviation & 18 & 24 & 32 & 28 & 3 & 1 \\
\hline Hand & extension digit 3 & 12 & 6 & 33 & 39 & 8 & 8 \\
\hline
\end{tabular}

\section{Local tenderness}

Tenderness at palpation of the lateral elbow region was moderate in 52 patients and severe in 29 patients. 
Table 4-5 lists the localization of the tendemess in the regions defined in the methods section (Figure 4-1)

Table 4-5.

Localization of tenderness at the lateral epicondyle

\begin{tabular}{lcc}
\hline Sites & Cyriax & Injection \\
\hline I & 4 & 0 \\
II & 38 & 46 \\
III & 7 & 5 \\
IV & 0 & 0 \\
V & 4 & 2 \\
\hline
\end{tabular}

Thus, 84 patients had maximal tenderness over the origin of the extensor carpi radialis brevis or the extensor digitorum.

\section{Grip strength}

The absolute value of grip strength depends on many variables and is not a good parameter of the severity of pain. We therefore used the increase or decrease in grip strength as the outcome parameter, which is why the grip strength found is described together with the grip strengths measured after six weeks (Table 4-14).

\subsubsection{Radiographic findings}

The radiographs of 13 patients showed calcification in the extensor origin at the lateral epicondyle. This calcification ranged from some minor calcifications to extensive calcification as shown in figure 4-3.

\subsubsection{Sedimentation rate}

The mean sedimentation rate in the first hour was $9( \pm 7)$. 


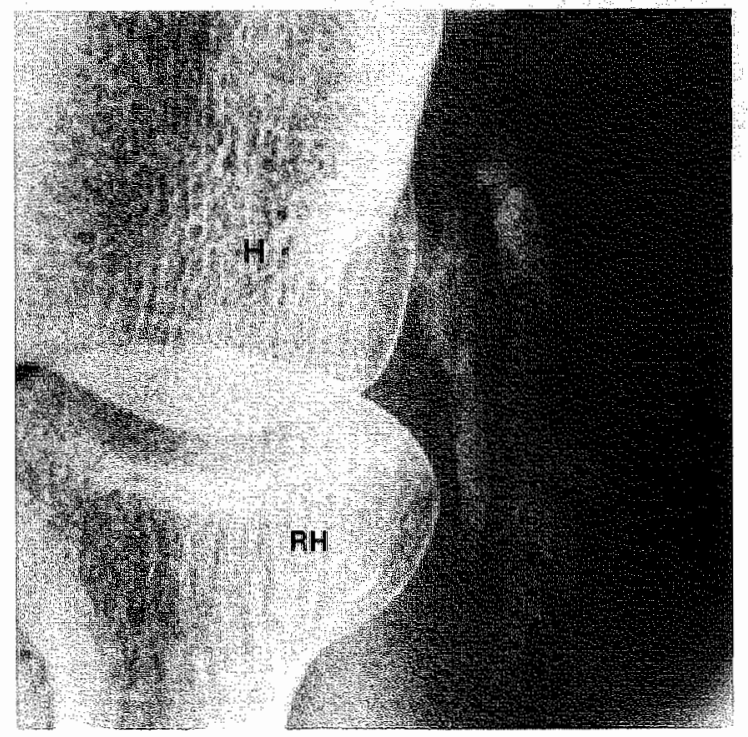

Figure 4-3.

A.P-radiograph of the elbow showing extensive calcification in the soft tissues near the lateral epicondyle of the right elbow.

Legend: humerus ( $\mathrm{H})$; radial head $(\mathrm{RH})$.

\subsubsection{Evaluation of treatment at 6 weeks}

The treatment results after six weeks could be evaluated in 103 of the 106 patients. Two patients from the physiotherapy group and one patient from the injection group were not examined after six weeks. Two of these three patients were evaluated after 12 weeks and the remaining patient was not seen again until the follow-up after fifty-two weeks.

One of the 53 patients discontinued the physiotherapy treatment because of severe pain during treatment. 20 of the 53 patients were treated with one injection, 17 patients received two injections and 16 patients had three injections.

\section{Severity of the pain}

The severity of pain decreased in both treatment groups after six weeks of treatment. Sixty-four patients (62\% of all patients) experienced little or no pain at the lateral epicondyle, compared to 3 patients (3\%) before treatment. Table 4-6 shows the treatment results in the physiotherapy group and the injection group. The patients treated with corticosteroid injections improved significantly better than the patients who had received physiotherapy according to Cyriax $(p<0.0001)$. 
Severity of pain at 0 weeks and 6 weeks for all patients, for the physiotherapy group (Cyriax) and for the injection group (Injection). Values are numbers

\begin{tabular}{lccccc} 
& \multicolumn{1}{c}{0 weeks } & \multicolumn{1}{c}{6 weeks } & & \\
\cline { 2 - 3 } \cline { 5 - 6 } Pain & All patients & Cyriax & Injection & All patients \\
\hline Absent & 0 & 3 & 22 & 25 \\
Slight & 3 & 19 & 20 & 39 \\
Moderate & 62 & 22 & 9 & 31 \\
Severe & 41 & 7 & 1 & 8 \\
Total & 106 & 51 & 52 & 103 \\
Missing & & 2 & 1 & 3 \\
\hline
\end{tabular}

Significance of difference: $\mathrm{X}^{2}$-test for linear trend $=23.3 ; \mathrm{p}<0.0001$

\section{Occurrence of pain}

After six weeks, $53 \%$ of all patients seldom or never experienced pain, compared with $9 \%$ of the patients before the beginning of treatment (Table 4-7). The improvement in the injection group was significantly higher than that in the physiotherapy group $(\mathrm{p}<0.0001)$.

Subjective loss of grip strength

At the six week follow-up, patients from both treatment groups said they experienced subjective loss of grip strength less often. Ninety patients had loss of grip strength at the start of the treatment, while 45 experienced loss of grip strength after a period of six weeks of treatment (Table 4-8). The results in the corticosteroid group were significantly better than those in the physiotherapy group ( $p=0.003$ ).

\section{Return to work at six weeks}

At six weeks, 13 of the 36 patients who were on sick leave had resumed their professional activities (Table 4-9). As result of the advised restriction of painful activities several patients in both groups refrained from professional activities until the follow-up at six weeks. No significant differences between the 2 treatment groups were noted. 
Table $4-7$.

Occurrence of pain at 0 weeks and 6 weeks for all patients, for the physiotherapy group (Cyriax) and for the injection group (Injection). Values are numbers

\begin{tabular}{|c|c|c|c|c|}
\hline \multirow[b]{2}{*}{ Occunrence } & \multirow{2}{*}{$\frac{0 \text { weeks }}{\text { All patients }}$} & \multicolumn{2}{|l|}{6 weeks } & \multirow[b]{2}{*}{ All patients } \\
\hline & & Cyriax & Injection & \\
\hline Never & 0 & 3 & 22 & 25 \\
\hline Seldom & 9 & 15 & 15 & 30 \\
\hline Regularly & 47 & 24 & 12 & 36 \\
\hline Often/ continuously & 50 & 9 & 3 & 12 \\
\hline Total & 106 & 51 & 52 & 103 \\
\hline Missing & & 2 & 1 & \\
\hline
\end{tabular}

Significance of difference: $\mathrm{X}^{2}$-test for linear trend $=17.5 ; \mathrm{p}<0.000 \rrbracket$

Table 4-8.

Subjective loss of grip strength at 0 weeks and 6 weeks for all patients, for the physiotherapy group (Cyriax) and for the injection group (Injection). Values are numbers

\begin{tabular}{|c|c|c|c|c|}
\hline \multirow[b]{2}{*}{ Loss } & \multirow{2}{*}{$\frac{0 \text { weeks }}{\text { All patients }}$} & \multicolumn{3}{|l|}{6 weeks } \\
\hline & & Cyriax & Injection & All patients \\
\hline Absent & 16 & 13 & 32 & 45 \\
\hline Slight & 23 & 24 & 13 & 37 \\
\hline Moderate & 53 & 12 & 5 & 17 \\
\hline Severe & $\llbracket 4$ & 2 & 2 & 4 \\
\hline Total & 106 & 51 & 52 & 103 \\
\hline Missing & & 2 & 1 & 3 \\
\hline
\end{tabular}

Significance of difference: $\mathrm{X}^{2}$-test for linear trend $=10.0 ; \mathrm{p}=0.003$ 
Tablo $4-9$

Resumption of labor at 0 weeks and 6 weeks for all patients, for the physiotherapy group (Cyriax) and for the injection group (Injection). Vallues are numbers

\begin{tabular}{|c|c|c|c|c|}
\hline & \multirow{2}{*}{$\frac{0 \text { wreks }}{\text { All patients }}$} & \multicolumn{3}{|c|}{6 weeks } \\
\hline & & Cyriax & Injection & All patients \\
\hline Resumed & 0 & 4 & 9 & 13 \\
\hline Still working & 31 & 14 & 15 & 29 \\
\hline Unable to work & 36 & 13 & 9 & 22 \\
\hline Not applicable & 39 & 20 & 19 & 39 \\
\hline Total & 106 & 51 & 52 & 103 \\
\hline Missing & & 2 & 1 & 3 \\
\hline
\end{tabular}

Significance of difference: $\mathrm{X}^{2}$-test for linear trend $=2.6 ; \mathrm{p}>0.05$

\section{Pain on resisted motion}

At the start of treatment, resisted dorsiflexion of the wrist was painful in all patients, while the pain was considered slight by 16 of 106 of them. At the six week follow-up 72 of the 103 patients had little or no pain at resisted dorsiflexion of the wrist (Table 4-10). The corticosteroid group had significantly less pain at resisted dorsiflexion than the physiotherapy group ( $<<0.0001$ ).

Resisted supination of the forearm showed a reduction of the pain provoked, which was significantly different for the two treatment groups ( $p=0.007$ ) (Table 4-11).

Resisted pronation of the forearms did not yield any significant differences between the 2 groups ( $p=0.39$ ) (Table not presented).

At six weeks, resisted extension of the middle finger was less painful in the injection group than in the physiotherapy group (Table 4-12).

\section{Local tenderness}

The tenderness at the lateral epicondyle decreased in the course of treatment. This decrease in tenderness was significantly greater in the injection group than in the physiotherapy group (Table 4-13). 
Table 4-10.

Pain provoked by resisted dorsiflexion of the wrist at 0 weeks and 6 weeks for all patients, for the physiotherapy group (Cyriax) and for the injection group (Injection). Values are numbers
0 weeks
6 weeks

Pain

All patients

Cyriax

Injection

All patients

Absent

0

5

26

31

Slight

16

24

17

41

Severe

90

22

9

31

Total

106

51

52

103

Missing

2

1

Significance of difference: $\mathrm{X}^{2}$-test for linear trend $=19.2 ; \mathrm{p}<0.0001$

\section{Table 4-11.}

Pain provoked by resisted supination of the forearm at 0 weeks and 6 weeks for all patients, for the physiotherapy group (Cyriax) and for the injection group (Injection). Values are numbers

\begin{tabular}{|c|c|c|c|c|}
\hline \multirow[b]{2}{*}{ Pain } & \multirow{2}{*}{$\frac{0 \text { weeks }}{\text { All patients }}$} & \multicolumn{3}{|l|}{ 6. weeks } \\
\hline & & Cyriax & Injection & All patients \\
\hline Absent & 56 & 36 & 47 & 83 \\
\hline Slight & 43 & 12 & 5 & 17 \\
\hline Severe & 7 & 3 & 0 & 3 \\
\hline Total & 106 & 51 & 52 & 103 \\
\hline Missing & & 2 & 1 & 3 \\
\hline
\end{tabular}

Significance of difference: $X^{2}$-test for linear trend $=7.328 ; p<0.0068$

\section{Localization of tenderness}

At six weeks, no tenderness could be elicited in 30 patients ( 26 patients from the corticosteroid group and 4 from the physiotherapy group). Sixty-five patients showed tenderness on location II (pain at the anterior aspect of the lateral 
Table 412 .

Pain prowoked by resisted extension of the middle finger at 0 weeks and 6 weeks for all patients, for the physiotherapy group (Cyriax) and for the injection group (Injection). Values are numbers

\begin{tabular}{|c|c|c|c|c|}
\hline \multirow[b]{2}{*}{ Paín } & \multirow{2}{*}{$\frac{0 \text { weeks }}{\text { All patients }}$} & \multicolumn{3}{|l|}{6 weeks } \\
\hline & & Cyriax & Injection & All patients \\
\hline Absent & 18 & 22 & 37 & 59 \\
\hline Slight & 72 & 23 & 15 & 38 \\
\hline Severe & 16 & 6 & 0 & 6 \\
\hline Total & 106 & $5 \rrbracket$ & 52 & 103 \\
\hline Missing & & 2 & 1 & 3 \\
\hline
\end{tabular}

Significance of difference: $X^{2}-$ test for linear trend $=11.1 ; p<0.0009$

epicondyle). The figures for the location I, III, IV and V were rather low, which made it impossible to find any relation between the localizations before the start of the treatment and the results of the treatment (Table 4-21).

\section{Grip strength}

The absolute value of the grip strength is known to be related to sex, profession, dominance of the arm, type of sports or hobby, and other variables. For this reason, we calculated the increase or decrease in grip strength at six weeks, and compared it with the pre-treatment values in the involved as well as the non-involved arm (Table 4-14). The increase in the grip strength in the injection group was higher than that in the physiotherapy group. The increase, however, only differed significantly when the grip strength was measured with the elbow in full extension. The painful arm showed a greater standard deviation than the control arm. Subjective improvement correlated very well with increase of grip strength.

\section{Patient's assessment at six weeks}

One third of the patients were dissatisfied with the treatment results after six weeks. Forty-eight per cent of the patients were satisfied with the improvement (Table 4-15). Satisfaction was lower in the physiotherapy group $(\mathrm{p}<0.0001)$. 
Table 4-13.

Tendemess at the lateral epicondyle at 0 weeks and 6 weeks for all patients, for the physiotherapy group (Cyriax) and for the injection group (Injection). Values are numbers

\begin{tabular}{lccccc} 
& \multicolumn{1}{c}{ Oweeks } & & & \\
\cline { 2 - 5 } Pain & All patients & Cyriax & Injection & All patients \\
\cline { 2 - 5 } & 0 & 4 & 26 & 30 \\
Absent & 24 & 21 & 14 & 35 \\
Slight & 52 & 21 & 11 & 32 \\
Moderate & 30 & 5 & 1 & 6 \\
Tevere & 106 & 51 & 52 & 103 \\
Missing & & 2 & 1 & 3 \\
\hline
\end{tabular}

Significance of difference: $\mathrm{X}^{2}$-test for linear trend $=19.2 ; \mathrm{p}<0.0001$

\section{Table 4-14.}

Increase (decrease) in grip strength in kilograms at 6 weeks in comparison to initial grip strength for the physiotherapy group (Cyriax) and the injection group (Injection). Values are numbers

Mean $\pm S D$

\section{Elbow in 0 degrees:}

Painful arm:

$\begin{array}{lr}\text { Cyriax } & 2.3 \pm 10.6 \\ \text { Injection } & 10.7 \pm 14.9\end{array}$

Control arm:

$\begin{array}{lll}\text { Cyriax } & & -0.6 \pm 8.7 \\ \text { Injection } & & -1.8 \pm 5.6\end{array}$

Elbow in 90 degrees:

Painful arm:
Cyriax
$3.1 \pm 10.2$
Injection
$4.9 \pm 10.6$

Control arm:

$$
p>0.05
$$

Cyriax

Injection 
Trable $4-15$.

Patient"s assessment at 6 weeks

\begin{tabular}{lcccc}
\hline Satisfaction & Cyriax & Injection & Total & $(\%)$ \\
\hline Dissatisfied & 26 & 9 & 35 & $(34)$ \\
Moderately Satisfied & 11 & 8 & 19 & $(18)$ \\
Satisfied & 14 & 35 & 49 & $(48)$ \\
& & 52 & 103 & $(100)$ \\
Total & 51 & 1 & 3 & \\
Missing & 2 & & & \\
\hline
\end{tabular}

Significance of difference: $\mathrm{X}^{2}$-test for linear trend $=17.5 ; \mathrm{p}<0.0001$

Overall result rating at six weeks

At six weeks, $49(47 \%)$ of the patients had a good or excellent result. 18 patients of the injection group and 1 patient of the physiotherapy group were completely free of symptoms (Table 4-16). Sixteen patients in the injection group and 38 in the physiotherapy group had a moderate or poor result. These differences between the treatment groups were significant with a $p$-value less than 0.0001 .

Table 4-16.

Overall result rating at: 6 weeks

\begin{tabular}{lcccc}
\hline & Cyriax & Injection & Total & $(\%)$ \\
\hline Excellent & 1 & 18 & 19 & $(18)$ \\
Good & 12 & 18 & 30 & $(29)$ \\
Moderate & 15 & 10 & 25 & $(24)$ \\
Poor & 23 & 6 & 29 & $(29)$ \\
& 51 & 52 & 103 & $(100)$ \\
Total & 2 & 1 & 3 & \\
Missing & & & & \\
\hline
\end{tabular}

Significance of difference: $\mathrm{X}^{2}$-test for linear trend $=25.4 ; \mathrm{p}<0.0001$ 
Multiple linear regression was used on the log-transformed values for grip strength. For the other outcome variables, multiple logistic regression was used.

Table 4-17 presents the coefficients from the final regression models: In the case of grip strength, these coefficients are to be interpreted as the amount of increase in the outcome parameter if the predictor rises by one unit, assuming the other predictors to be fixed. Thus, the 'treatment' entry means that the "log grip strength at week 6 was 0.156 higher in the injection group than in the Cyriax group, assuming patients were identical with respect to the other predictors. Since grip strengths were analyzed as $\mathrm{c}$ log transformed, this means that the mean grip strength measured in full extension of the elbow was $16.8 \%$ higher in the injections group. For the categorized outcome variables, the coefficients are the e $\log$ odds ratios of outcome levels $j+1$ or higher relative to levels 1 up to $j$ for a one unit increase in the predictor variable.

Table 4-17.

Coefficients from multiple regression analysis

Outcome parametera

$\begin{array}{lllll} & \text { grip } & 90^{\circ} \text { grip overall patient's } & \text { Predictor } & \text { strength strength } \\ \text { result satisfaction of pain loss of grip tenderness }\end{array}$

\begin{tabular}{|c|c|c|c|c|c|c|c|}
\hline $0^{\circ}$ grip strength & 0.427 & $0.569^{\mathrm{b}}$ & . & -1.09 & . & . & \\
\hline Treatment & 0.156 & $0.011^{\mathrm{c}}$ & -2.048 & 1.446 & -2.046 & -1.448 & -1.771 \\
\hline Dominance & . & . & . & . & . & . & · \\
\hline Severity of pain & . & . & . & . & 0.543 & . & 0.627 \\
\hline Cervical complaints & 0.232 & 0.208 & . & 0.989 & . & . & -0.928 \\
\hline \multicolumn{8}{|l|}{ Subjective loss grip } \\
\hline strength & . & . & . & . & . & 0.504 & . \\
\hline Tenderness & . & . & . & . & . & . & . \\
\hline \multicolumn{8}{|l|}{ Resisted wrist } \\
\hline dorsiflexion & 0.227 & 0.214 & . & . & . & . & . \\
\hline \multicolumn{8}{|l|}{ Resisted extension } \\
\hline of digit 3 & . & . & 0.626 & . & . & . & . \\
\hline
\end{tabular}

a) The table gives coefficients from the reduced models; coefficients not entered (except treatment) were not significant; ${ }^{b}$ ) For $90^{\circ}$ grip strength, the corresponding 0 weeks $90^{\circ}$ value was used as a predictor; ${ }^{\mathfrak{c}}$ ) Not significant. 
Table 4-17 shows that the treaiment is the most important factor determining outcone parameters. With the exception of the grip strength measured with the elbow in 90 degrees of flexion, the 2 types of treatment differed on all outcome parameters presented in table 4-17. Patients from the corticosteroid group had a better result on these outcome parameters than patients treated with Cyriax" method. Patients with a good grip strength (measured with an extended elbow at the start of treatment) seemed to be less satisfied with the treatment results at six weeks than patients with a poor grip strength.

If the patient had cervical complaints, this negatively influenced the assessment of the treatment results by the patient at six weeks (i.e., more patients were dissatisfied with the outcome of treatment). Furthermore, local tenderness of the epicondyle at six weeks was also less reduced in these patients (Table 4-17).

The severity of the pain provoked by resisted dorsiflexion of the wrist at week 0 was related to grip strength at six weeks. If dorsiflexion provoked more severe pain, this corresponded with a greater increase in grip strength than if less pain was provoked.

A more painful resisted dorsiflexion of the middle finger seemed to be related to a better result of treatment, based on the overall result rating. No significant relation with other parameters was found.

\subsubsection{Additional treatments between 6 and 52 weeks}

After the assessment of the treatment results at six weeks several patients needed additional treatment. If spontaneous pain and the pain provoked by resisted dorsiflexion of the wrist were absent or slight and the patient was satisfied, no further treatment was given. If there was some improvement in the severity of pain and the resisted dorsiflexion of the wrist, the original conservative treatment was continued. Conservative treatment was altered in unimproved cases. If treatment had been entirely unsuccessful operative treatment was proposed to patients who had had a long period of complaints ( $>6$ months) and extensive conservative treatment ( $>3$ months). Table $4-18$ gives an outline of the treatment of the patients after the initial six weeks.

Twenty-seven patients of the Cyriax group did not require further treatment after the initial six weeks. Of these 27 patients 4 returned to the out-patient clinic with a recurrence of symptoms. In the steroid injection group 40 patients did not require further treatment after six weeks, but 16 patients returned because of recurring of symptoms. In both treatment groups 2 patients had a recurrence of 
Table 4-18.

Outline of treatment given at 6 weeks. Randomized treatment was stanted at 0 weeks. The figures are numbers of patients

$>6$ weeks

Further conservative treatment

No further

$0-6$ weeks

treatment Operation

Cyriax

Injection

Cyriax

27

Injection

40

10

7

9

8

3

2

symptoms after twelve weeks of treatment. The interval between the start of the treatment and the recurrence was 15 weeks in the injection group (SD: 6.4 weeks). In the physiotherapy group this period was 19 weeks (SD: 8.5 weeks) (Figure 4-4). With the exception of three patients who received treatment according to Cyriax, the other 13 patients with recurrent symptoms were treated conservatively with local corticosteroid injections.

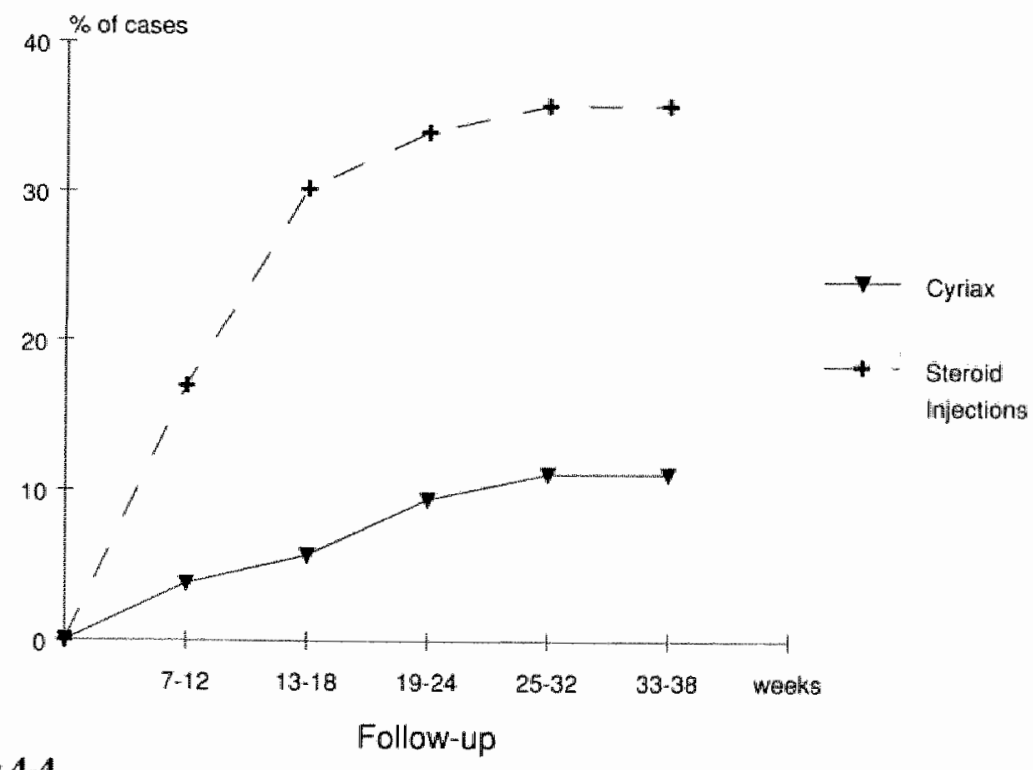

Figures 4-4.

Follow-up

Cumulative recurrences after successful conservative treatment for the injection group and the physiotherapy group. 
Thus, six weeks of treatment sufficed in half of our patients ( 23 in the physiotherapy group and 24 in the corticosteroid group). At fifty-two weeks, 27 patients had only received physiotherapy treatment and 29 patients only corticosteroid injections, as determined by the randomization. Seventeen patients of the steroid group and 14 from the physiotherapy group were operated upon. Statistical analysis showed no differences between these two operated groups on any of the preoperative variables.

\subsubsection{Evaluation of treatment at 52 weeks}

All patients were seen for follow-up fifty-two weeks after initiation of treatment, with the exception of one man (from the injection group) who had died. We analyzed our patients group on the basis of the initial randomization ('intention to treat' principle). This analysis showed no differences between the 2 randomized treatment groups (Table 4-19). The p-values in this table are based on the $\mathrm{X}^{2}$-test for linear trend.

Table 4-19.

Differences between the 2 treatment groups at 52 week follow-up

p-value

Severity of pain

$0.41 *$

Occurrence of pain

$0.63 *$

Subjective loss of grip strength

$0.18 *$

Tenderness

$1.00 *$

Resisted extension of the wrist

$0.58 *$

Resisted supination

$0.45 *$

Resisted pronation

$0.78 *$

Resisted extension of the middle finger

$0.43 *$

Patient"s assessment

$0.20 *$

Overall result rating

$0.17 *$

* = not significant

Therefore, the two treatment groups will be discussed as one group in the further analysis. This analysis represents the one-year results of our treatments. The findings at the fifty-two week follow-up were compared with the findings at the start of treatment and at six weeks: 

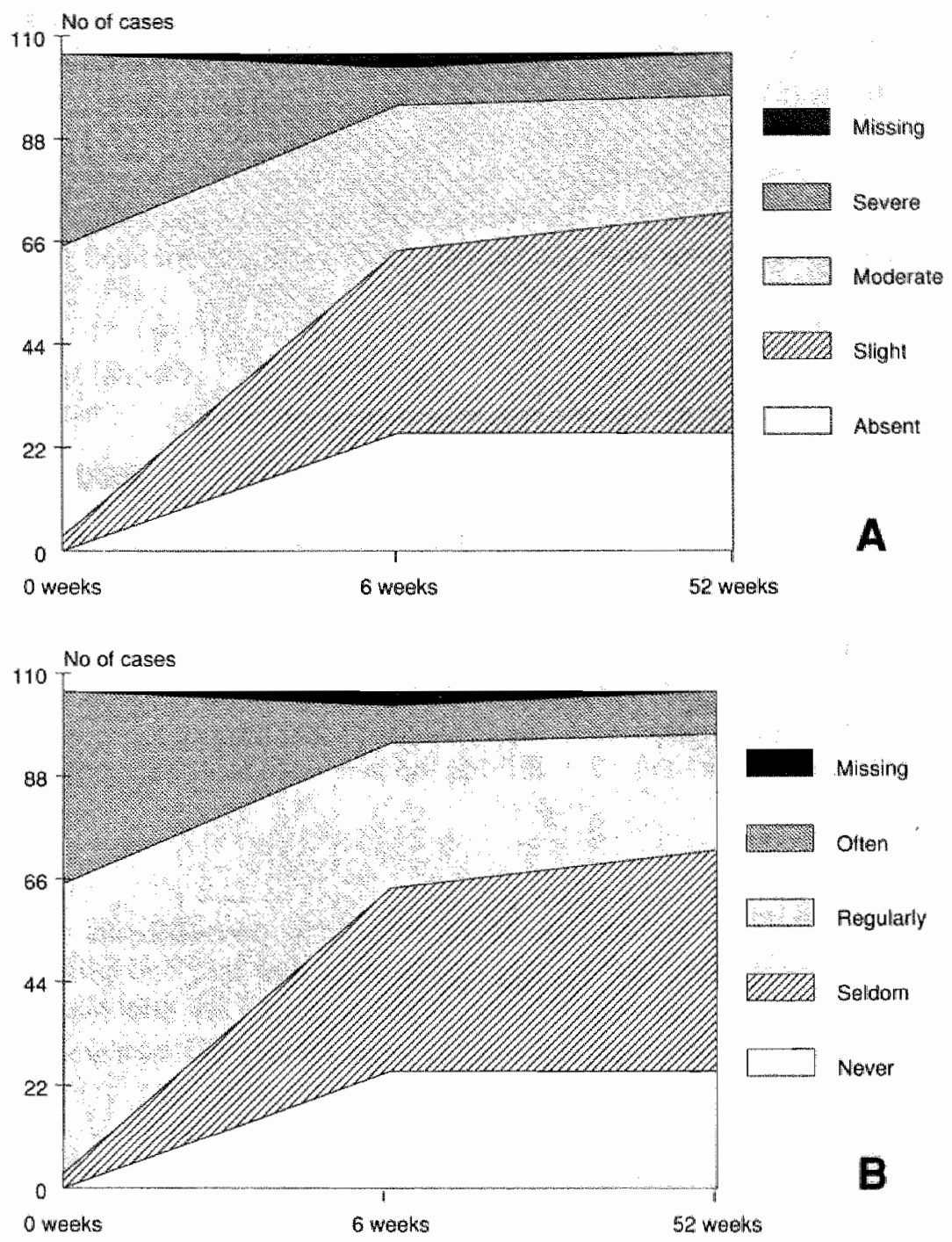

Figure 4-5.

Follow-up

The severity (A) and the occurrence of pain (B) at 0,6 and 52 weeks for the 2 treatment groups.

Severity and occurrence of pain

At one year, 72 patients had little or no pain at the lateral epicondyle (Figure 4-5-A). Twenty-five patients were free of symptoms at fifty-two weeks, the same number as had been recorded at six weeks. In the period between six weeks and fifty-two weeks, however, the severity of pain decreased; slight pain persisted in 39 patients at six weeks and in 47 patient at fifty-two weeks. 
The occurrence of pain showed a similar pattern as the severity of pain (Figure 4-5-B). At fifty-two weeks 65 patients $(61 \%)$ had seldom or no pain compared to 55 patients $(52 \%)$ at six weeks.

Subjective loss of grip strength

58 of the 103 patients seen at the evaluation at six weeks had subjective loss of grip strength. After one year, 55 patients (52\%) still experienced loss of grip strength (Figure 4-6).

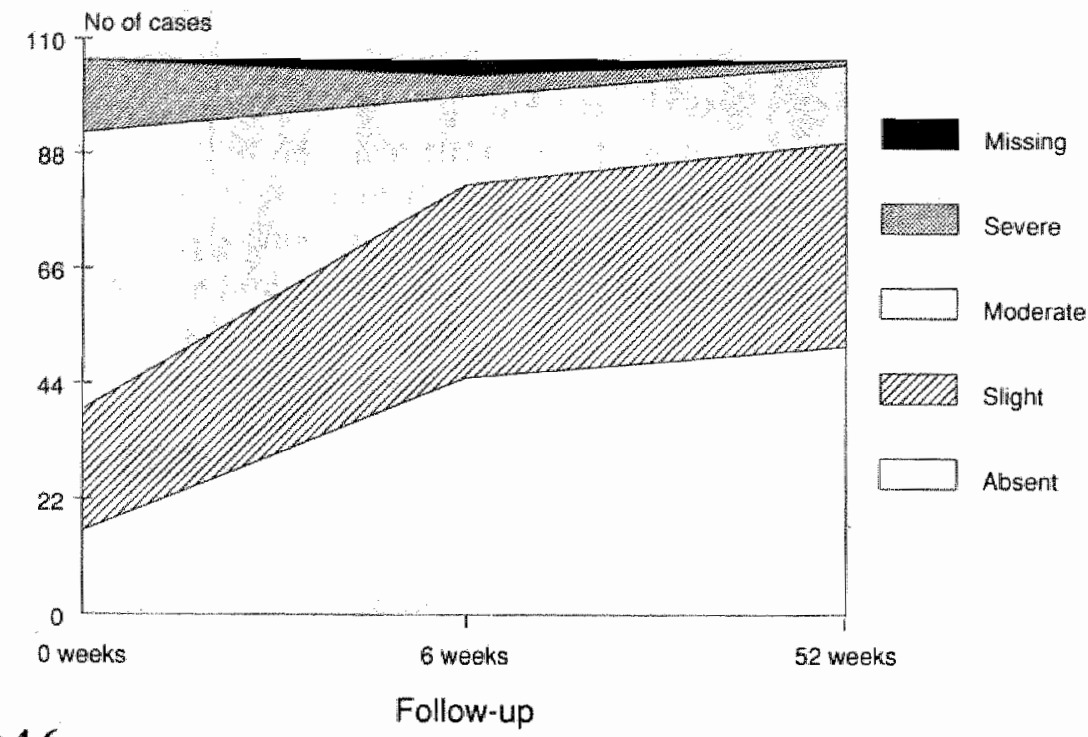

Figure 4-6.

The subjective loss of grip strength at 0,6 and 52 weeks for the 2 treatment groups.

Return to work

At the beginning of treatment 67 patients had a job. At the evaluation after one year, 57 of these 67 (84\%) were performing their original professional activities (Table 4-20). Two patients had a new job and 8 patients were receiving a disability pension, were waiting for one, or had decided to leave their job because of persisting elbow symptoms.

\section{Pain on resisted motion}

At fifty-two weeks, resisted dorsiflexion of the wrist did not provoke pain at the lateral epicondyle in 39 patients (37\%) (6 weeks: 31 patients) (Figure 4-7-A). At fifty-two weeks, resisted dorsiflexion caused severe pain in 10 patients ( 6 weeks: 31 patients). The resisted extension of the middle finger at fifty-two weeks showed a pattern similar to the pattern found at six weeks (Figure 4-7-B). 
Table 4-20.

Return to work at 52 weeks

\begin{tabular}{lccc}
\hline & 0 weeks & 6 weeks & 52 weeks \\
\hline Back at work & 0 & 13 & \\
& 31 & 29 & 57 \\
Still at work & 36 & 22 & 8 \\
Not working (sick leave) & 39 & 39 & 37 \\
Not applicable (no job) & - & 0 & 4 \\
New Profession & 106 & 103 & 106 \\
\end{tabular}

The pain provoked by resisted pronation and supination showed only slight differences between the evaluation at one year and that at six weeks; resisted supination was not painful in 87 patients at one year, compared to 83 patients at six weeks. Resisted pronation was not painful in 91 patients, both at six and at fifty-two weeks.

\section{Tenderness}

At both six and fifty-two weeks, the lateral epicondyle was not tender in 30 patients. At the one year follow-up the severity of the tenderness had decreased in comparison with the figures at the six week follow-up (Figure 4-8).

\section{Localization of tenderness}

27 patients ( $25 \%$ ) did not experience local tenderness over the lateral epicondyle at the one year follow-up. Table 4-21 lists the localizations of tenderness. No major differences were found between the patterns of localization and the findings at 0 and 6 weeks, except for an increased tenderness over the muscles of the forearm in 9 patients.

\section{Grip strength}

The grip strength increased over the one year period, but in contrast to the six week measurements (Table 4-14) no differences between the treatment groups were seen. At one year, the mean grip strength (with extended elbow) of the treated arm was 33.1 (SD \pm 13.5 ) in the corticosteroid group and 34.5 (SD \pm 14.6 ) in the Cyriax group. The control arm showed a grip strengths for the two groups of $36.6(\mathrm{SD} \pm 15.6)$ and $38.8(\mathrm{SD} \pm 19.6)$ respectively. 

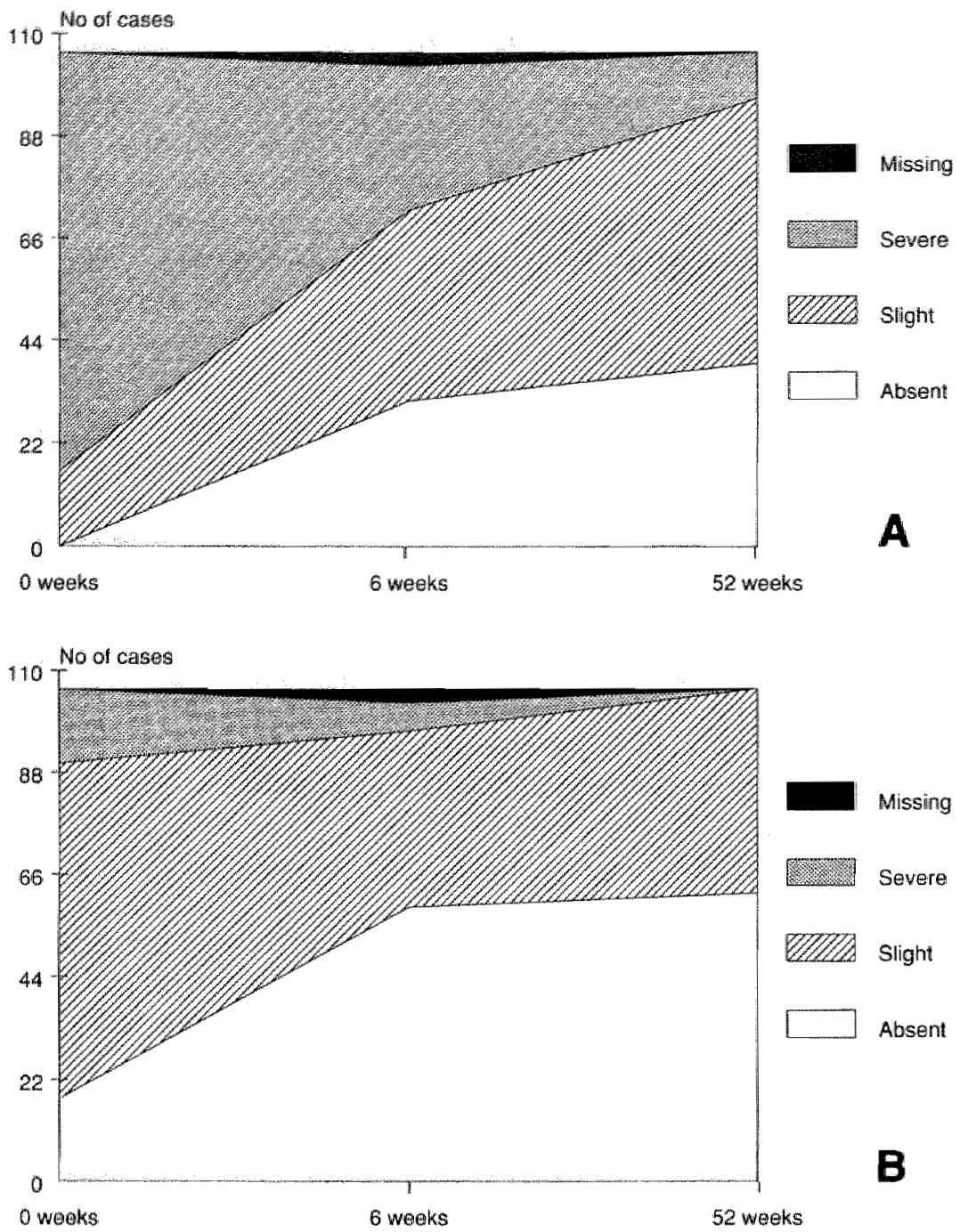

\section{Figure 4-7.}

Follow-up

The resisted dorsiflexion of the wrist (A) and the middle finger (B) at 0,6 and 52 weeks for the 2 treatment groups. 


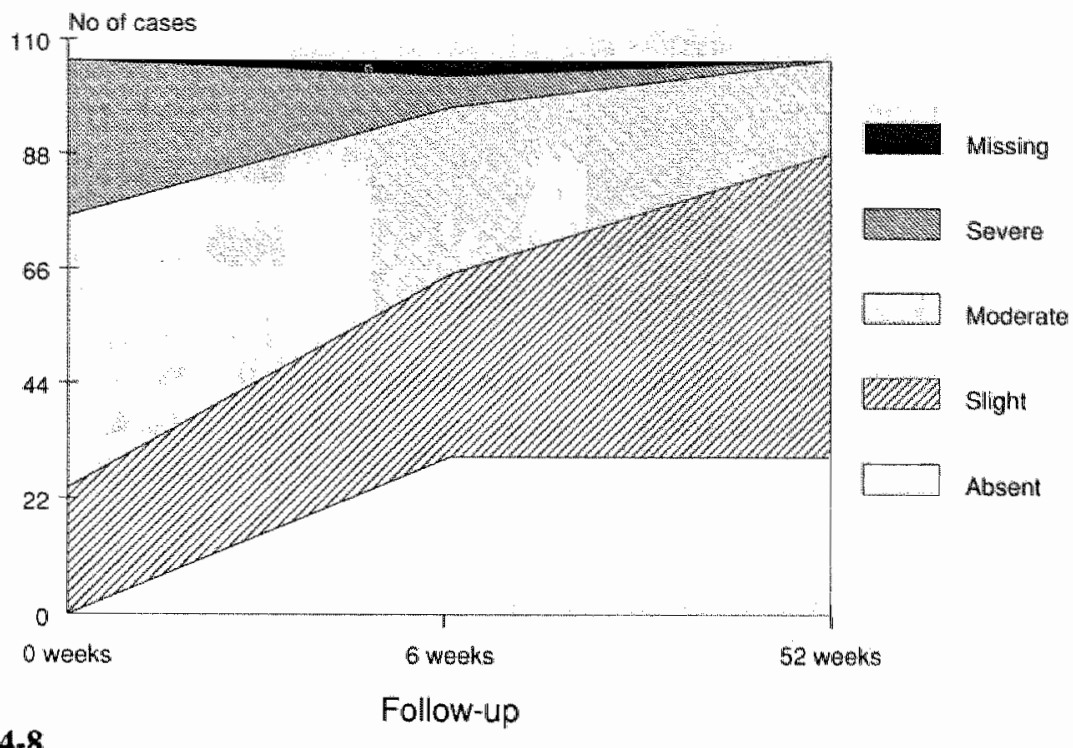

\section{Figure 4-8.}

The tenderness of the lateral epicondyle at 0,6 and 52 weeks for the 2 treatment groups.

\section{Table 4-21.}

Localization of tenderness at the lateral epicondyle at 0 weeks, 6 weeks and 52 weeks

\begin{tabular}{lccc}
\hline Region & 0 weeks & 6 weeks & 52 weeks \\
\hline I & 4 & 2 & 0 \\
II & 84 & 65 & 60 \\
III & 12 & 4 & 8 \\
IV & 0 & 0 & 9 \\
V & 6 & 2 & 2 \\
No tenderness & 0 & 30 & 27 \\
\hline
\end{tabular}

Before the start of the treatment the grip strengths with an extended elbow were $18.4(\mathrm{SD} \pm 9.3)$ in the corticosteroid group and $23.3(\mathrm{SD} \pm 13.8)$ in the Cyriax group. The control arm showed a grip strengths in the two groups of 36.3 (SD \pm 13.7) and 37.8 ( $S D \pm 14.0$ ). This means that the grip strength had increased considerably at one year compared with the start of the treatment, but was not yet normal. 

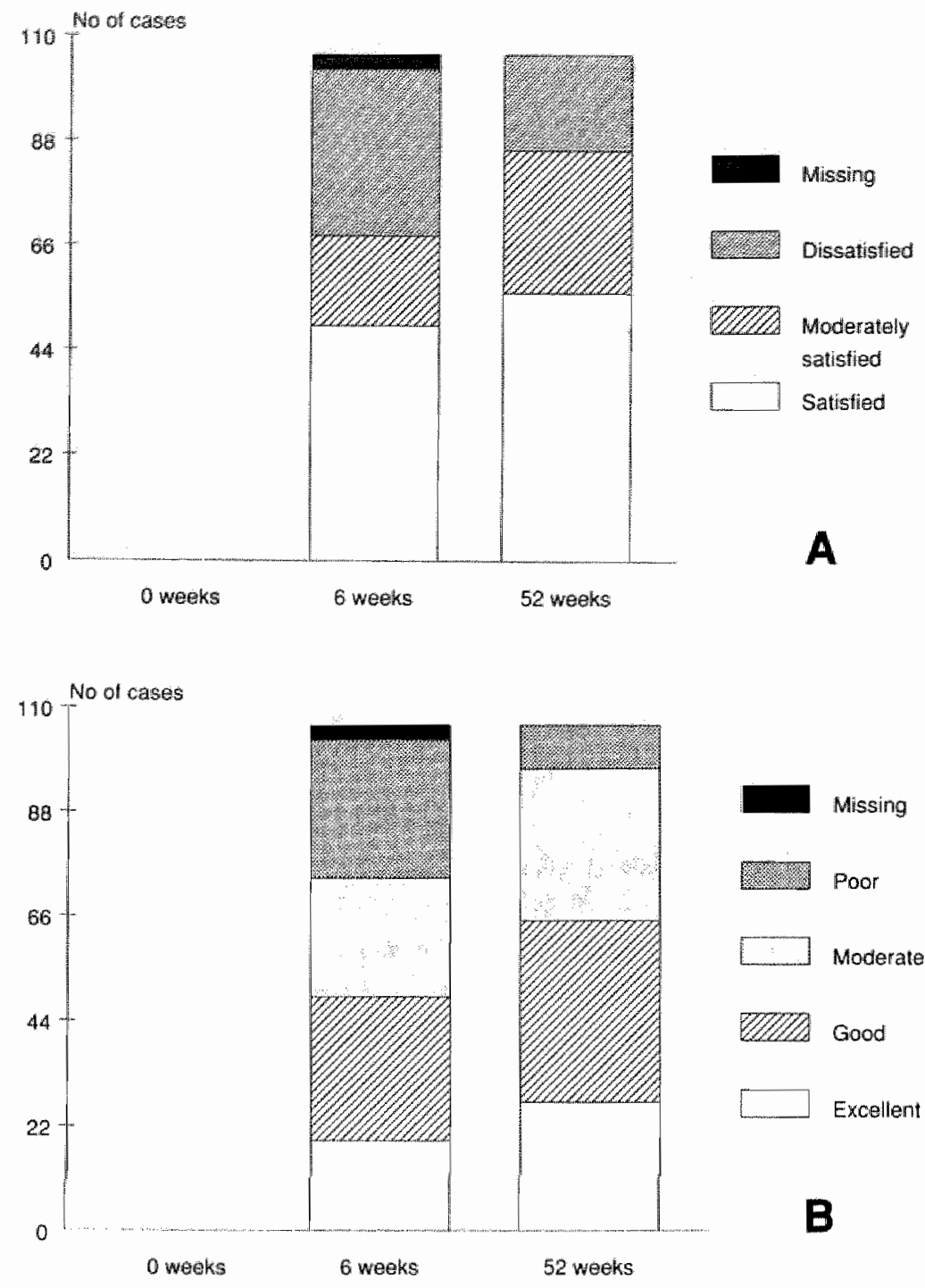

\section{Figure 4-9.}

Follow-up

Patients' assessments of the results of treatment (A) and overall results (B) at 0,6 and 52 weeks for the 2 treatment groups.

\section{Patient's assessment}

Fifty-six patients were satisfied with the treatment results (53\%). At six weeks 49 patients had been satisfied (48\%) (Figure 4-9-A). Thirty patients were moderately satisfied at one year $(28 \%)$. Twenty patients were dissatisfied at fifty-two weeks ( 6 weeks: 35 patients). 
Overall result rating at 52 weeks

One year after initiation of treatment, one quarter of the patients had an excellent result (Figure 4-9-B). These patients were fully recovered and had no pain. However, the result of $39 \%$ of the patients was considered moderate or poor.

\section{Side-effects}

No side-effects of either treatment were seen at the follow-up after six and fifty-two weeks. No infections occurred after injections and no hypopigmentation of the skin was induced by the corticosteroid injections.

\section{Statistical analysis of outcome of treatment at 52 weeks}

Multiple regression was used to obtain a more accurate evaluation of the therapy results and to identify other predictors for the outcome at fifty-two weeks.

Grip strength (both in full extension and in 90 degrees of flexion), overall result rating, patient's satisfaction, severity of pain, subjective loss of strength and local tenderness at fifty-two weeks were used as outcome parameters.

Predictors at week 0 were dominance of the elbow, grip strength with an extended elbow, pain, cervical complaints, subjective loss of grip strength, local tenderness, and pain at resisted extension of the wrist and the middle finger. The randomized therapy (injections versus Cyriax) was not considered because at fifty-two weeks no uniform treatment had been used. For the rest, the method was identical to the procedure described for the statistical analysis of the outcome of treatment at six weeks.

Table 4-22 shows that no predictors of the end result of treatment after fifty-two weeks could be identified. A high grip strength with an extended elbow correlated with a high grip strength value at fifty-two weeks.

At fifty-two weeks, cervical complaints correlated with a reduced grip strength at 90 degrees of flexion of the elbow. A relation was also found between resisted dorsiflexion of the wrist and grip strength at 90 degrees of elbow flexion. Patients with slight pain at the start of treatment had greater grip strength at 90 degrees of flexion after fifty-two weeks than patients with more severe pain. All other parameters did not to correlate with each other. 


\section{Oatcome parametera}

$\begin{array}{llllll} & 0^{\circ} \text { grip } 90^{\circ} \text { grip overall: patient's } & \text { severity subjective local } \\ \text { Predictor } & \text { strength strength result satisfaction pain } & \text { loss of grip tenderness }\end{array}$

$0^{\circ}$ grip strength $\quad 0.430 \quad 0.549^{b}$.

Dominance

Severity of pain

Cervical complaints .

Subjective loss

grip strength

Tenderness

Resisted wrist

dorsiflexion

0.230

Resisted extension

of digit 3

a) The table gives coefficients from the reduced models; coefficients not entered were not significant; b) For $90^{\circ}$ grip strength, the corresponding 0 week $90^{\circ}$ value was used as a predictor.

\subsection{DISCUSSION}

The results of this study showed a significant improvement in the entire group of patients after a treatment period of six weeks. We further found that the results of corticosteroid injections were superior to those of the physiotherapeutic regimen of Cyriax at the six week follow-up. $69 \%$ of the patients who received corticosteroid injections had excellent or good results and $67 \%$ of them were satisfied with the treatment results. In the physiotherapy group, $27 \%$ of the patients had excellent or good results and $26 \%$ were satisfied with the results of treatment.

The success rate in the injection group $(69 \%)$ was somewhat lower than the results reported in the literature. $92 \%$ of the patients studied by Day et al. (1978) improved or were cured with corticosteroid injections. Hughes and Currey (1969) claimed a success rate of $95 \%$ and Valtonen (1967) reported a success rate of $86 \%$. Our results in the physiotherapy group (27\%) are also somewhat lower than the results of treatment obtained by other authors, who reported a 
success rate for physiotherapy of between $29 \%$ (Binder et al. 1985) and $53 \%$ (Devereaux et al. 1985). In his thesis, Jonquière (1986) found no differences between the results of physiotherapy and the treatment according to Cyriax for shoulder and elbow disease. His group of patients with a tennis elbow was, however, too small to draw definite conclusions. In our study no other physiotherapeutic treatment was used to compare with the Cyriax 'treatment. However, the results of treatment we have found do not provide arguments to assume that this treatment is greatly superior to other types of physiotherapy (Devereaux et al. 1985, Chard and Hazleman 1988, Lundeberg et al. 1988).

After an initially successful six weeks of treatment, a considerable number of our patients experienced recurrence of the pain in the subsequent months. $34 \%$ of patients treated with corticosteroid injections showed recurrent symptoms. Most studies of steroid injections with a short follow-up have claimed excellent results of this treatment (Hohl 1961, Valtonen 1967, Hughes and Currey 1969, Day et al. 1978). Clarke and Woodland (1975) and Nevelös (1980), however, extending their follow-up to six months, also noticed a high recurrence of symptoms, which was as high as $66 \%$ in one group of their patients. Thus, corticosteroid injections may alleviate the pain in tennis elbow very well, but may not address the cause of the symptoms. The high recurrence rate may imply that patients should be instructed to avoid undue strain on the arm for some months, particularly if they feel they have been fully cured by the injections. The recurrence rate of $34 \%$ in the corticosteroid group contrasted with a rate of $11 \%$ in the physiotherapy group. On the other hand, the initial success rate of the treatment in the physiotherapy group was much lower, which means that fewer patients could experience a recurrence of symptoms.

Our study was set up as a randomized trial. Clinical trials may be flawed by various types of biases (Gartland 1988). In our study, the investigator both provided the treatment and evaluated the results and for that reason observer bias was inevitable introduced (Laupacis et al. 1989). In addition, it was not feasible to blind the patients for the type of treatment received. However, every assessment of outcome of treatment was done without knowledge of prior evaluations or the type of treatment (except operative treatment). In addition, by measuring grip strength we attempted to use a more objective outcome parameters

The most characteristic feature of tennis elbow is pain over the lateral epicondyle resulting in tenderness in a limited area over the lateral epicondyle (Trethowan 1929, Cyriax 1936, Lamphier et al. 1959, Goldie 1964, Cyriax 1982). Another typical sign of tennis elbow is that resisted dorsiflexion of the clenched wrist provokes pain in the area of the lateral epicondyle where the local tenderness was elicited (Goldie 1964). In our study, the selection of patients was 
based on localized epicondylar pain that increased at resisted dorsiflexion of the wrist. The aim of these restrictive inclusion criteria was to prevent atypical lateral elbow pain being diagnosed as tennis elbow. For that reason no patients with isolated pain in the proximal part of the muscle bellies of the forearm extensors were included. As a result, the conclusions of this study cannot be generalized to all patients with lateral elbow pain.

The typical pain pattern found in our patients was a dull pain around the lateral epicondyle, which in some cases radiated down the forearm and increased during contraction of the forearm extensors. However, there were several exceptions to this classic pattern; $39 \%$ of our patients also had pain radiating to the upper arm. Thirteen per cent of patients had more pain during rest than during activities and $56 \%$ experienced pain at night.

24 of the 106 patients had cervical complaints, which proved to have a negative influence on the outcome of treatment at six weeks. This influence was not found at the fifty-two week follow-up. At fifty-two weeks, however, cervical complaints were related to a lower grip strength at 90 degrees of flexion of the elbow. At other moments during the study, however, the grip strength in this position showed no relation with tennis elbow. We think the relation found between cervical complaints and lower grip strength might be explained by generalized pain in the muscles of the forearm. None of the other anamnestic pretreatment variables had any significant influence on the treatment result.

Half of our patients considered their professional activities to be responsible for their complaints. This is in agreement with the cornmon view that the main causes of tennis elbow are over-use or repetitive activities during profession, hobbies or housework (Binder and Hazleman 1983). This rather high percentage also suggests that patients in whom elbow complaints limit professional activities are more often referred by the general practitioner than other patients.

Pain may be influenced by many factors and is difficult to measure. Therefore, we measured grip strength with a hand dynamometer and used this as a more objective parameter for the outcome of treatment. Gripping is mainly carried out by the forearm flexors. The power grip, however, requires a fixed wrist joint in 15 degrees of dorsiflexion (Van Gemert 1984). This action of the forearm extensors generates tensile forces at the common extensor origin, which is painful in tennis elbow (Snijders et al. 1987). Thus, grip strength measurements in tennis elbow register primarily the severity of pain and may serve as a quantitative measure of the response to treatment (Goldie 1964, Nirschl 1979, 
Thurtle et al. 1984). The Jamar hand dynamometer has been recommended as the best measure for grip strength because it gives reproducible results and is very accurate (Schmidt and Toews 1970, Mathiowetz et al. 1984).

In our study, measuring the grip strength proved to be very useful as an outcome parameter, comparing the grip strength before the start of treatment with the grip strength at follow-up. Flexion of the elbow was found to influence the outcome of the grip strength measurements with the hand dynamometer. Gripping with the elbow in full extension produced more pain than gripping with the elbow in 90 degrees of flexion. This difference has not been reported by other authors and is not fully understood. It may be theorized that the ventral part of the tendinous origin of the forearm extensors presses on the lateral epicondyle at full extension of the elbow. However, this phenomenon might also be explained by an increased tension on the common extensor origin, due to changes in length.

There were considerable differences in grip strength among the patients. In 83 patients the dominant arm - which usually has a higher grip strength than the non-dominant arm - was involved. Other authors have reported significant differences in grip strength between males and females and between people with different professional or recreational activities (Schmidt and Toews 1970, Thorngren and Werner 1979, Sheehan et al. 1983, Mathiowetz et al. 1984). These influences were, however, eliminated by using only the increase or decrease of grip strength as the outcome parameter. The standard deviation in the increase or decrease of the grip strength was higher for the involved elbow than for the uninvolved elbow. This is due to the fact that patients with unsuccessful treatment had a lower grip strength than patients with successful treatment. In the uninvolved elbow this phenomenon did not influence the measurement.

As for the resisted movements, two tests proved to be quite useful: dorsiflexion of the wrist and extension of the third finger. The other tests were only painful in some patients. The painful dorsiflexion of the wrist is generally accepted as a characteristic test for tennis elbow. Painful resisted extension of the middle finger, however, has been considered by several authors as a test typical of the so-called radial tunnel syndrome (Roles and Maudsley 1972). Two-thirds of our patients with typical tennis elbow, however, had pain during resisted extension of the middle finger. This means that this test cannot differentiate between tennis elbow and 'radial tunnel syndrome'. The extension of the third finger was more frequently painful than that of the other fingers. This is easily explained by the concept of the Lateral Cubital Force Transmission System (chapter 2). The extensor digitorum consists of 4 separate musculotendinous units. The musculotendinous unit of the extensor of the middle finger attaches proximally 
directly distal to the lateral epicondyle. The muscle fibres of the other extensors of the fingers attach more distally to the walls of the sheath around the extensor digitorum muscle fibres.

Radiographs of our patients showed calcifications in the soft tissues near the lateral epicondyle in 13 patients $(12 \%)$. The $95 \%$-confidence interval for this percentage lies between 6.4 and $22.7 \%$ (Rümke 1976). Other authors have found similar percentages of calcifications on radiographs (Table 1-2) (Hohl 1961, Ilfeld and Field 1966, Baumgard 1982, Waldis 1989). We have made radiographs of all our patients, while other studies are unclear on that point. Only Gondos (1958) and Goldie (1964) reported a higher percentage of calcification. A significant correlation between the calcification on radiographs and the severity of complaints was not found in our patients.

Many physicians regard tennis ellbow as a benign self-limiting condition, improving with or without treatment within 8 to 12 months (Cyriax 1936, Quin and Murly 1954, Baily and Brock 1957). Our study underlines, however, that for a selected group of patients tennis elbow may be the source of prolonged elbow pain and persistent impairment of activities for many months. The duration of complaints may exceed a period of one year and the economic consequences for some patients may be very high. Half of our patients with a job could not carry out their professional activities at the time when they were referred. After one year of treatment, a high percentage of patients still experienced pain at the lateral epicondyle. Nine per cent still experienced severe pain, while $68 \%$ of patients felt pain intermittently. Only $23 \%$ of patients were completely free of pain. These results are comparable to those of the study by Binder and Hazleman (1983). Twenty-three per cent of their patients experienced severe pain one year after treatment. Fifty-seven per cent felt intermittent pain and $28 \%$ of their patients were completely free of pain. It must be stressed, however, that our study group consisted of patients referred by the general practitioner. Thus, our patients formed a selection of all patients with a tennis elbow. The orthopaedic out-patient clinic serves a population of between 150,000 and 200,000 people for common orthopaedic disorders such as tennis elbow. Over a one-year period we have diagnosed tennis elbow in 138 patients (described in this Chapter and in Chapter 5). This means that the incidence at our out-patient clinic was between 0.07 and $0.09 \%$ of the general population.

The high percentage of patients with complaints persisting after one year seems to be disappointing. However, the severity of complaints was significantly reduced after one year of treatment. A considerable number of the patients with persistent complaints considered these complaints not severe enough to require further therapy. Fifty-three per cent of our patients were fully satisfied with the 
results of treatment. Nineteen per cent were dissatisfied with the outcome. These findings underline the necessity of adequate follow-up in studying the treatment of tennis elbow.

Of the patients who participated in the trial, $29 \%$ were operated upon because of persistent symptoms. This figure seems to be rather high in comparison to other studies of conservative treatment, in which 5 to $10 \%$ of patients were treated operatively (Coonrad and Hooper 1973). Many of these studies, however, come from countries where there is free admission to medical specialists. In the Netherlands most people are obliged to go to the general practitioner, who is to decide whether referral is necessary. Our study in general practice showed that less than $17 \%$ of the patients with tennis elbow were referred by the general practitioner (Chapter 2). Combining these data, it can be calculated that some five per cent of all patients who sought medical help in the study period underwent operative treatment. This figure is comparable with those found in other studies.

One year after the start of treatment no differences were found between the groups initially randomized. At fifty-two weeks, $50 \%$ of the entire group of patients had only received the initial treatment, $30 \%$ of all patients had been operated and $20 \%$ had received combination therapy. This mixing of treatment groups may have induced the lack of differences between the two groups of patients. The initial treatment was not found to influence the outcome of treatment after one year.

If all modalities of treatment for a disorder are equally effective, the procedure of choice should be the one that is the least invasive, least expensive and most time efficient (Kamien 1990). Our study showed that local corticosteroid injections gave better relief of pain in a shorter period of time than the physiotherapy method described by Cyriax. The high recurrence rate after corticosteroid injections, however, implies that the effect of the injections may be transient and they may only produce symptomatic relief. The injection therapy was, however, more time efficient than physiotherapy; 2 additional visits to the out-patient clinic for the injection group compared to 12 visits to the physiotherapy department for the physiotherapy group. No important side-effects of either method were found in our study group.

In view of the time efficiency, the satisfactory level of pain relief and the lack of side effects encountered we tend to prefer the treatment with corticosteroid injections in cases in which rest alone does not provide adequate relief of the tennis elbow complaints. After six weeks, corticosteroid injections are found to be more effective than Cyriax"s treatment, although their healing effect is uncertain. 


\section{5.}

It may be concluded from this study that:

- the results of corticosteroid injections in tennis elbow were superior to the physiotherapeutic regimen according to Cyriax at the six week follow-up.

- 34\% of the patients treated with corticosteroid injections experienced a recurrence of symptoms, as opposed to $11 \%$ of the patients treated with physiotherapy. After correction for unsuccessful cases the recurrence rates were identical.

- one year after the start of treatment, $75 \%$ of patients still had complaints, although most of them did not consider these severe enough to require further therapy.

- cervical pain complaints proved to have a significant negative influence on the outcome of treatment at six weeks.

- resisted dorsiflexion of the middle finger cannot differentiate between tennis elbow and 'radial tunnel syndrome'.

- measuring grip strength with a hand dynamometer proved to be very useful at follow-up, if initial grip strength was compared with grip strength at follow-up. The highest sensitivity was reached if the measurements were done with an extended elbow.

\section{APPENDIX}

\section{Multiple regression analysis}

Multiple regression analysis is used to explain variations in the outcome parameter from variations in the predictors. The result obtained has the form

$$
\text { outcome }=\text { constant }+b_{1} * X_{1}+\ldots+b_{k} * X_{k}=\text { residual }
$$

Here, $X_{1} \ldots X_{k}$ are the values of the predictors considered, and $b_{1} \ldots b_{k}$ are determined so as to minimize the standard deviation of the residual or, in other words, to maximize the correlation of $b_{1} * X_{1}+. .+b_{k} * X_{k}$ with the outcome. This coefficient of correlation is called the multiple correlation $R$; its square $R^{2}$ is called the proportion explained variance. The higher $R^{2}$, the more accurate 
predictions of the week 6 results are possible on the basis of week 0 parameters. The p-values of individual regression coefficients reflect whether the prediction becomes significantly worse when the predictor under consideration is omitted from the equation. For instance, grip strength with the elbow in 90 degrees of flexion at week 0 is a significant predictor of grip strength with the elbow in 90 degrees of flexion at week 6 . However, therapy is not a significant predictor of grip strength with the elbow in 90 degrees of flexion at week 6 .

For measurements on a continuous scale, such as grip strength, the analysis method does justice to the nature of the data and gives readily interpretable results. For outcomes on a two-, three- or four-point scale, however, the linear regression model which assumes a normally distributed continuous outcome variable, is at variance with the data. The more elaborate ordinal logistic regression model was used. In this model, the probability of each possible outcome (e.g., excellent, good, moderate or poor for the overall result rating) is considered to be a function of the pretreatment variables and of the randomized treatment. More precisely, when $1 . . \mathrm{k}$ are the categories of the outcome $\mathbb{Y}$, the model is, for each $\mathrm{j}=1 \ldots \mathrm{k}-1$

$$
\log \frac{\operatorname{Prob}(Y>j)}{1-\operatorname{Prob}(Y>j)}=\alpha_{j}+\beta_{\rfloor} x_{1}+\ldots+\beta_{x} x_{x}, j=1 \ldots k-1
$$

This means that the contribution to the $(\log )$ odds of category $j+1$ or higher of a prognostic factor is the same for each level j, but the baseline-value $\alpha_{j}$ (the value when all prognostic variables are zero) of these odds may be different for each $j$. The $\beta$ 's are the log odds ratios of outcome levels $j+1$ or higher relative to levels 1 to $\mathrm{j}$ for a one unit increase in the $\mathrm{X}$ predictor variable. For example, the log odds of "less than good" in the overall result rating are 2.05 lower for the patients treated with corticosteroids, implying an odds ratio of 0.13 . The a's represent the relative frequencies of the summed ( $j+1$ to $k$ ) outcome categories. If there are only two outcome categories, formula (1) reduces to the classical logistic regression model:

$$
\log \frac{\operatorname{Prob}(Y=1)}{1-\operatorname{Prob}(Y=1)}=\alpha+\beta_{1} x_{1} \ldots \beta_{p} x_{p}
$$





\section{CHAPTER}

\section{Operative treatment of tennis elbow}

a prospective study of the results of the lateral release operation

This chapter has been accepted for publication in the Journal of Bone and Joint Surgery (Am). 


\section{OPERATIVE TREATMENT OF TENNIS ELBOW}

a prospective study of the results of the lateral release operation

\subsection{INTRODUCTION}

If conservative treatment of tennis elbow has failed, operative treatment may be considered. Many authors have found it difficult to define the position of operations in the treatment scheme of tennis elbow. In a condition seen so often (Chapter 3), which in many cases does not even require the attention of a physician (Chapter 3) and which seems to have a self-limiting nature or responds rather well to conservative treatment (Chapter 3 and 4), many hesitate to undertake an operation. However, some patients may recover very slowly and their tennis elbow may be the source of prolonged elbow pain and persistent impairment of activities over a long period of time (Chapter 4). Moreover, patients with a job which requires the full use of the arms, may loose it if they are unable to work for many months (Coonrad and Hooper 1973, Boyd and McLeod 1973, Nirschl and Pettrone 1979). Thus, operative treatment is generally considered to be indicated if complaints last too long or are too severe. Both factors are greatly influenced by the needs of the patient from an economic point of view or by his ambitions in sports or other leisure activities.

Chapters 3 and 4 discussed the results of conservative treatment. In this chapter we describe the results of operative treatment of a group of patients in a non-randomized trial. The group consisted of patients seen at the out-patient clinic with a tennis elbow, diagnosed on the basis of the following criteria: pain at the lateral side of the elbow, tenderness over the forearm extensor origin, and 
pain at the lateral epicondyle during resisted dorsiflexion of the wrist with the elbow in full extension. Sixty-three patients entered this study and were followed prospectively for five years.

\subsection{PATIENTS AND METHODS}

\subsubsection{Selection of patients}

Over a 12 month period (July 1984 - July 1985) all patients who had operative treatment for tennis elbow were included in this study. All patients were operated upon because of pain persisting for more than six months, which interfered with normal activities in their profession or daily life and which was not or inadequately alleviated by conservative treatment. The decision to operate also depended upon the individual patient's functional requirements. For that reason patients in whom tennis elbow complaints interfered with professional activities more often asked for operative treatment than patients with interference of hobby or sports. Patients who had had previous operations at the lateral side of the elbow were excluded from the study. Patients with neurological disorders such as carpal tunnel disease or cervical radicular compression were allso excluded.

The group studied consisted of 63 patients. Thirty-one of these did not participate in the conservative trial because of exclusion criteria for this randomized trial or refusal to participate. The most important reason for exclusion was the number of corticosteroid injections (more than three) which the patients had already received from their general practitioner. The other 32 patients were failures from the conservative trial. Details of the preoperative characteristics of the patients are presented in table 5-1. The mean age of the patients was 44.6 years. The mean period of conservative treatment was 50 weeks (standard deviation 30 weeks). Prior conservative treatment included corticosteroid injections and physiotherapy as well as several other treatment modalities (Table $5-2$ ). The conservative treatment has been performed by the general practitioner and/or by the orthopaedic surgeon at the out-patient clinic.

Sixty-two of the 63 patients were evaluated six weeks and one year after operation. The remaining patient, a man, had died of cardiovascular disease. At the five-year follow-up two more patients had died. In addition, three patients could not be evaluated: one could not be located and two patients refused to return to the out-patient clinic for follow-up. Thus, 57 of the 63 patients were evaluated five years after operation. 
Table 5-1.

Preoperative chatacteristics of the operated group

W

Sex:

$\begin{array}{lll}\text { Male } & 42 & (67) \\ \text { Female } & 21 & (33)\end{array}$

Side:

$\begin{array}{lll}\text { Right } & 41 & (65) \\ \text { Left } & 22 & \text { (35) }\end{array}$

Conservative treatment:

$<26$ weeks 22

$26-52$ weeks 33

$>52$ weeks 8

Extension of the elbow:

Normal $\quad 56$

Limited

7

Localization of maximal tenderness:

Extensor origin

58

Radiohumeral join

4

Dorsal side of the epicondyle

Radiograph of the elbow:

Normal

Calcifications

9

\section{Table 5-2.}

Prior conservative treatment of the operated patients $(\mathrm{N}=63)$

\begin{tabular}{ccc}
\hline & Yes & No \\
\hline $\begin{array}{c}\text { Physiotherapy } \\
\text { \#eneral Practitioner }\end{array}$ & 25 & 38 \\
\#rthopaedic Surgeon & 20 & 43 \\
Corticosteroid injections & & $16 *$ \\
\# General Practitioner & 47 & $30 * *$ \\
Orthopaedic Surgeon & 33 & \\
\hline
\end{tabular}

* mean: 2.5 injections ; ** mean: 1.4 injections 
The operation was performed as an out-patient procedure by one surgeon using a standard protocol. The skin and deeper tissues overlying the lateral epicondyle were infiltrated with lidocaine $1 \%$ with adrenaline 1:100,000. A gently curved incision approximately 5 centimeters long (extending from $1 \mathrm{~cm}$ proximal to the lateral epicondyle to $4 \mathrm{~cm}$ distal to this structure) was made over the lateral epicondyle. The extensor origin was divided transversely close to its origin at the lateral epicondyle allowing the origin to retract distally (Figure 5-1).

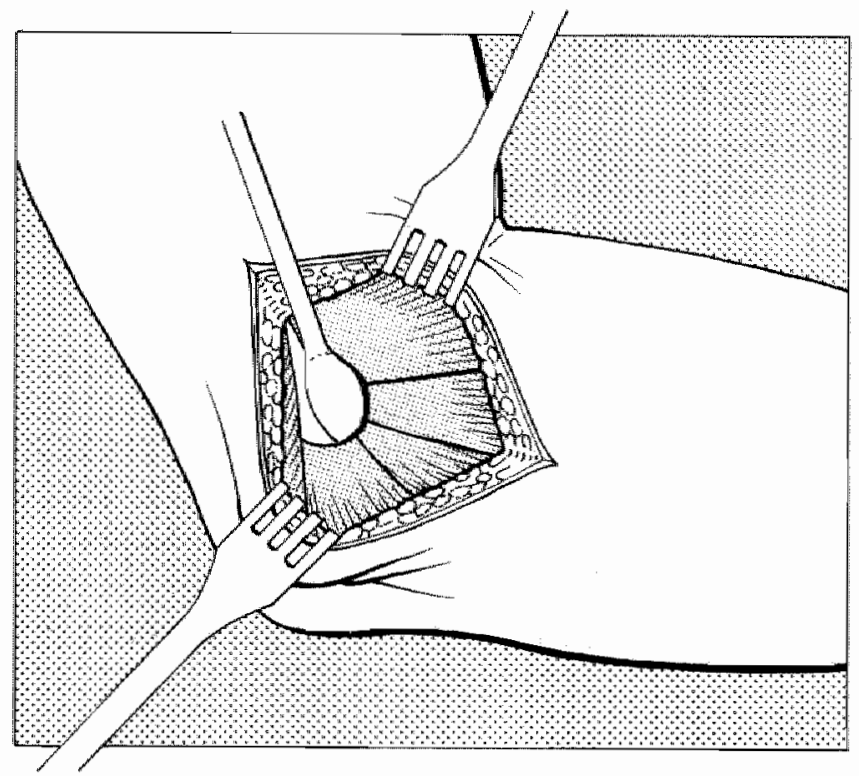

Figure 5-1.

Schematic representation of the operative technique.

Since the patients retained muscle control, they were asked to dorsiflex the wrist, which allowed evaluation of the completeness of the release. Attention was paid to dividing the medioventrall part of the intermuscular septum between the extensor carpi radialis brevis muscle and the extensor digitorum muscle (See chapter 2). The extensor origin was detached from the lateral epicondyle until the synovium of the radio-humeral joint was visible. A small incision through the synovium of the elbow was always made in order to inspect the radial humeral joint for intra-articular pathology. Specimens were taken from the tissue of the proximal extensor tendon in all patients as well as biopsies of the synovium and of any synovial fringe if the synovium seemed to be abnormal. 
When the wound was closed, only the subcutaneous tissues and the skin were sutured. Postoperative care included a light compression bandage and sling used for two weeks. Patients were encouraged to extend the elbow several times a day, but were advised not to use the arm for other activities. Two weeks after operation the skin sutures were removed and the patients were instructed to avoid undue stress on the arm for another four weeks.

\subsubsection{Evaluation of the results of treatment}

The patients were evaluated 6 weeks, 12 months and 5 years (range 50-65 months) after operation. The investigator both provided the treatment and evaluated the results. He was, however, blinded for prior evaluations.

Outcome parameters were: severity and occurrence of pain, subjective loss of grip strength, epicondylar tenderness, pain at resisted motion of elbow, wrist and fingers, patient's assessment and the overall result rating (see Table 4-2). Patients were asked to grade these parameters by choosing from a set of 3 or 4 items (see Table 4-1).

Grip strength was measured with a Jamar ${ }^{\circledR}$ digital hand dynamometer. During each test the patient was unable to see the display. The grip strengths of both hands were measured in kilograms. Measurements were performed with the elbow fully extended and at 90 degrees of flexion. For each position the average of two measurements was recorded as the strength. In addition, patients were questioned as to their ability to resume their occupation.

The assessment made by the patient consisted of the following items:

- Satisfied

- Moderately satisfied

- Dissatisfied

For the overall ratings of the results of treatment made by the examining physician the following criteria were used: 


Excellen:
Good:
- Complete relief of pain
- No pain provoked by resisted dorsiflexion of the wrist
Moderate:
- Improved; essentially no pain at night and slight pain at rest;
discomfort after strenuous activities on a more tolerable level
- Slight pain provoked by resisted dorsiflexion of the wrist
- No improvement
- Moor:
wrist
- Decreased grip strength

Several preoperative factors were correlated with the postoperative outcome.

\subsubsection{Statistics}

All data are given as numbers of patients, with the exception of the grip strengths, which were expressed as means $\pm \mathrm{SD}$. The paired T-test was used to compare changes in grip strength before treatment and at six weeks, one year and five years after treatment. $\mathrm{X}^{2}$-test for linear trend was used to determine the relationship between pre-treatment variables and the outcomes of treatment (Cochran 1954). P-values $<0.05$ were considered to be statistically significant.

\subsubsection{Histology of extensor origin}

Specimens were taken from the tissue of the proximal extensor tendon in all patients. Biopsies of the synovium or the synovial fringe were only performed if the synovium seemed to be abnormal. After fixation in $4 \%$ buffered neutral formalin, samples were embedded in paraffin and cut into slices with a thickness of $5 \mu \mathrm{m}$ (microns). The sections were stained with hematoxylin and eosin. The descriptions of the microscopy were made by the Department of Pathological Anatomy of the Academic Hospital Maastricht. 


\subsubsection{Findings at operation}

In five patients, white material was found subcutaneously with no apparent associated changes in the surrounding tissues. Histology of this white material revealed an amorphous substance, which was believed to be a residual of the local injection of a corticosteroid preparation. In all but six patients the extensor origin was macroscopically normal. Of these six patients three had a partial superficial rupture of the origin. The extensor origin of two other patients had a shiny, edematous appearance. A small cyst was present in the origin of one patient. One patient had a rupture in the overlying fascia of the anconeus muscle. Nine patients had a rather large radiohumeral synovial fringe in comparison to the average size of this fringe. In three of them the fringe was more reddish in colour than the rest of the synovium. A moderate degree of chondromalacia of the radial head was seen in three patients, but no chondromalacia of the capitulum humeri was identified.

\subsubsection{Histology of extensor origin}

Routine sections from the proximal part of the common extensor origin showed vascular proliferation in 29 specimens ( $46 \%$ ) in the form of islands of small blood vessels between the collagen fibres. Associated with this increased vascularity there was an increase in the number of round nuclear cells as well as a fibroblastic reaction. Mucoid degeneration was noticed in 17 specimens (26\%). Components of an inflammatory reaction such as granulocytes, lymphocytes and plasma cells were virtually absent. Focal calcifications were found in 7 specimens $(11 \%)$. Chondroid metaplasia was found in 4 patients. Sections of 4 radiohumeral synovial fringes showed no abnormalities except for some calcification in one.

\subsubsection{Postoperative complications}

In one patient a wound infection developed and staphylococcus aureus was cultured from the wound. Antibiotic treatment was effective and no arthritis or synovial effusion developed. In no patients synovial fistula developed. Six weeks after operation, 14 patients showed a limitation of the extension of the 
elbow, which was never more than 20 degrees compared to the normal elbow. Physiotherapy was prescribed for 10 of these 14 patients because of this limited extension of the elbow.

\subsubsection{Results of treatment}

62 of the 63 patients were evaluated after six weeks. 62 patients were evaluated after 12 months and 57 patients were evaluated after an average of 58 months (range 50-65 months).

At six weeks 37 patients had either no pain or slight pain at the lateral epicondyle, whille at one year 47 patients $(76 \%)$ were free of pain and at five years 52 patients $(91 \%)$ were pain-free. Twenty-five patients $(40 \%)$ still had moderate to severe pain six weeks after operation. At the one-year follow-up 15 patients (24\%) still had moderate or severe pain. Five years after the operation 5 patients (9\%) described the severity of pain as moderate or severe (Figure 5-2 A and B).

Before operation all but three patients had noted some loss of grip strength. Forty-seven patients rated their loss as slight or moderate. At six weeks followup most patients were improved and at one year 41 patients regarded their grip strength as normal, while most of the remaining patients described only a slight reduction of grip strength (Figure 5-3). At the five-year follow-up 47 patients $(82 \%)$ assessed their grip strength as normal.

One year after operation, resisted extension of the wrist no longer provoked pain in $37 \%$ of the 62 evaluated patients $(60 \%)$ and this number had increased to 44 patients $(77 \%)$ at five years (Figure 5-4).

Preoperatively, resisted extension of the middle finger was painful in 49 (78\%) patients. This number had been reduced to 14 patients $(23 \%)$ one year postoperatively and to 8 patients $(14 \%)$ five years after surgery. Painful resisted supination decreased from $32(50 \%)$ preoperatively to, respectively, $11(18 \%)$ and 7 patients $(9 \%)$ after one and five years.

Six weeks after operation, epicondylar tenderness at palpation was present in 48 patients $(77 \%)$. After one year this number had decreased to 27 patients. However, at five years after surgery there was still tenderness over the lateral epicondyle in 23 patients ( $40 \%$ ) (Figure 5-5). This tenderness was most evident over the site of the transverse division of the origin (ventral surface of the lateral 

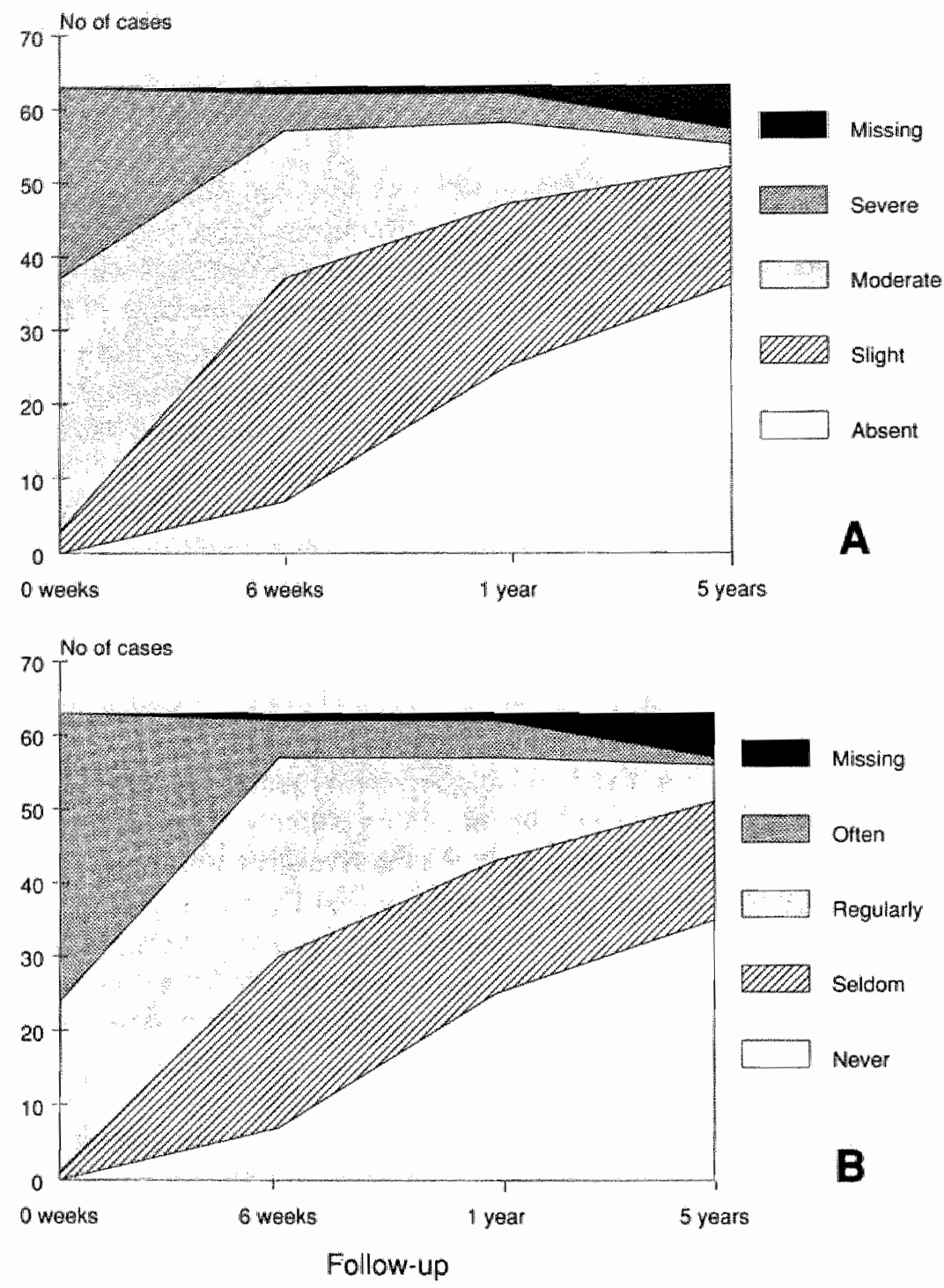

\section{Figure 5-2.}

Severity of pain (A) and occurrence of pain (B) preoperatively, at 6 weeks, at 1 year and at 5 yearis.

epicondyle). In 12 of these 23 patients, however, resisted dorsiflexion of the wrist did not provoke pain. No relation was found between epicondylar tenderness and the patient's assessment or the overall result rating. 


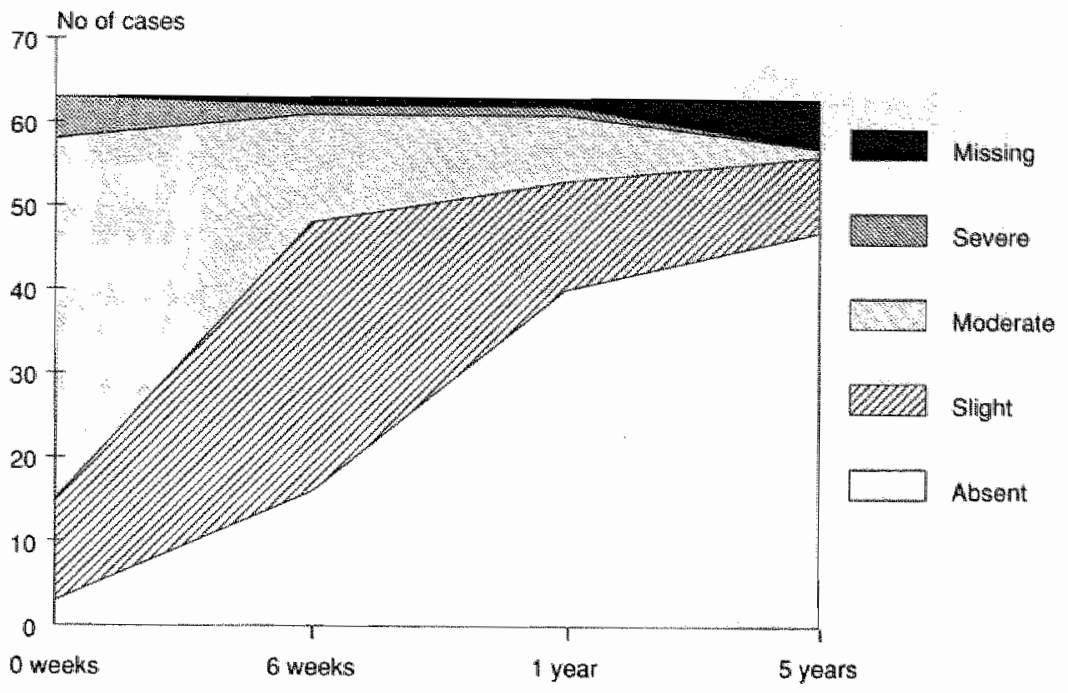

Figure 5-3.

Follow-up

Subjective loss of grip strength preoperatively, at 6 weeks, at 1 year and at 5 years.

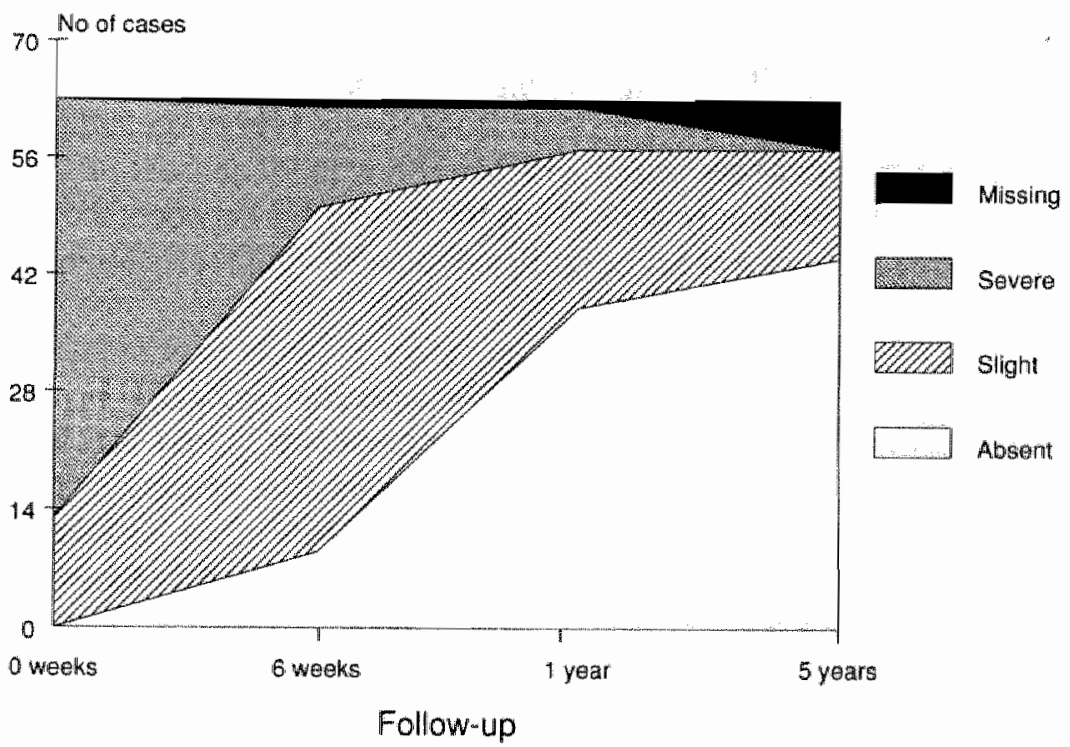

Figure 5.4.

Pain on resisted extension of the wrist preoperatively, at 6 weeks, at 1 year and at 5 years. 


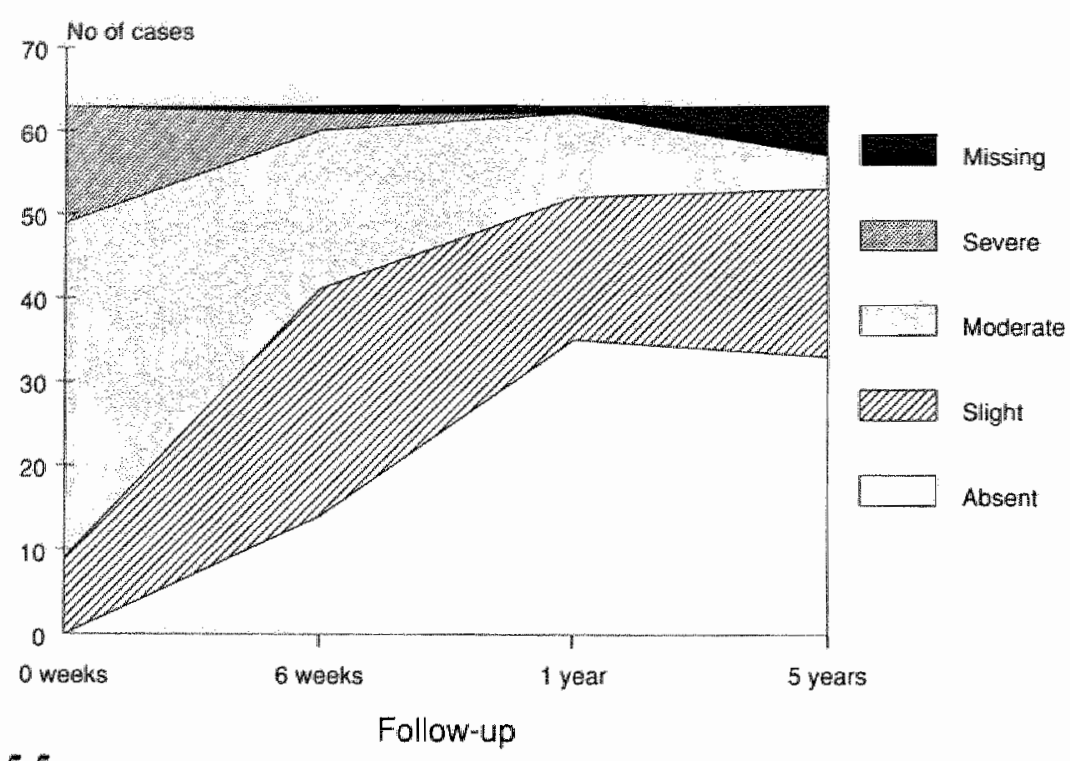

Figure 5-5.

Epicondylar tenderness at 6 weeks, at $]$ year and at 5 years.

The grip strength of the operated arm increased significantly after operation, as is shown in table 5-3. If the grip strength was measured with the elbow in full extension, this increase was significant at six weeks follow-up and at fifty-two weeks. Grip strength as measured with the elbow in 90 degrees of flexion also increased, but this was only significant for the period between six weeks and fifty-two weeks. There were no significant changes in the grip strengths of the control arms at any time.

At the follow-up examination one year after operation, 41 patients $(66 \%)$ were satisfied with their results of treatment. Eight patients (13\%) considered their results to be unsatisfactory (Figure 5-6). Five years after operation, 52 of the 57 patients $(91 \%)$ were satisfied with the results of treatment, while 2 patients were still dissatisfied with the outcome of treatment of their tennis elbow because of persistent pain.

Six weeks after operation only five patients were fully recovered. One year postoperatively 31 patients $(50 \%$ ) had an excellent result and 17 patients a good result, while 10 patients had a moderate result and 4 patients had a poor result (Figure 5-7). At the follow-up examination after five years, these figures had changed only slightly: $33(59 \%)$ patients had an excellent result, $18(32 \%)$ a good result, $5(9 \%)$ a moderate and $1(2 \%)$ a poor result. 
Table 5-3.

Grip strength at 0 weeks and at 6 weeks, 1 year and 5 years postoperatively (mean $\pm \mathrm{SD}$ ) in kgs

Painful arm Control arm

\section{Elbow in 0 degrees}

0 Weeks

$$
18.8 \pm 11.5
$$

$35.0 \pm 13.5$

Difference 0-6 weeks

4.8

1.5

6 Weeks

$23.6 \pm 11.0$

$36.5 \pm 13.5$

Difference 6-52 weeks

$11.8 * * *$

2.8

1. Year

$35.4 \pm 13.6$

$39.3 \pm 17.9$

0.9

$-3.5$

5 Years

Difference $1-5$ years

$36.3 \pm 12.5$

$35.8 \pm 14.7$

\section{Elbow in 90 degrees of flexion}

0 Weeks

$19.8 \pm 11.0$

2.0

$21.8 \pm 9.7$

$9.1 *$

$30.9 \pm 11.5$

0.1

$31.0 \pm 10.6$
$32.0 \pm 12.4$

1.5

$33.5 \pm 12.2$

0.9

$34.3 \pm 12.0$

$-1.1$

$33.2 \pm 13.4$

5 Years

Difference $1-5$ years:

** $p<0.001 ; * * * p<0.0001$

\section{Recurrences}

At the follow-up examination after one year and five years none of the patients had experienced a recurrence of symptoms after a pain-free interval. Most patients described some, albeit moderate improvement of complaints in the period between one and five years

\section{Return to work}

Before operation, 32 of the 44 patients who had paid employment were unable to work because of their tennis elbow (Table 5-4). Six weeks after operation, 8 of them had returned to work. Most of the others resumed their professional activities within six to twelve weeks and at the one year follow-up, 33 patients were performing their preoperative professional activities. Ten patients ( 5 because of persisting elbow complaints) were receiving a disablement benefit (WAO). Seven patients seen at the five year follow-up received disablement benefits because of their elbow complaints. However, at the time of the final follow-up two of the seven said they never experienced pain and the other five 


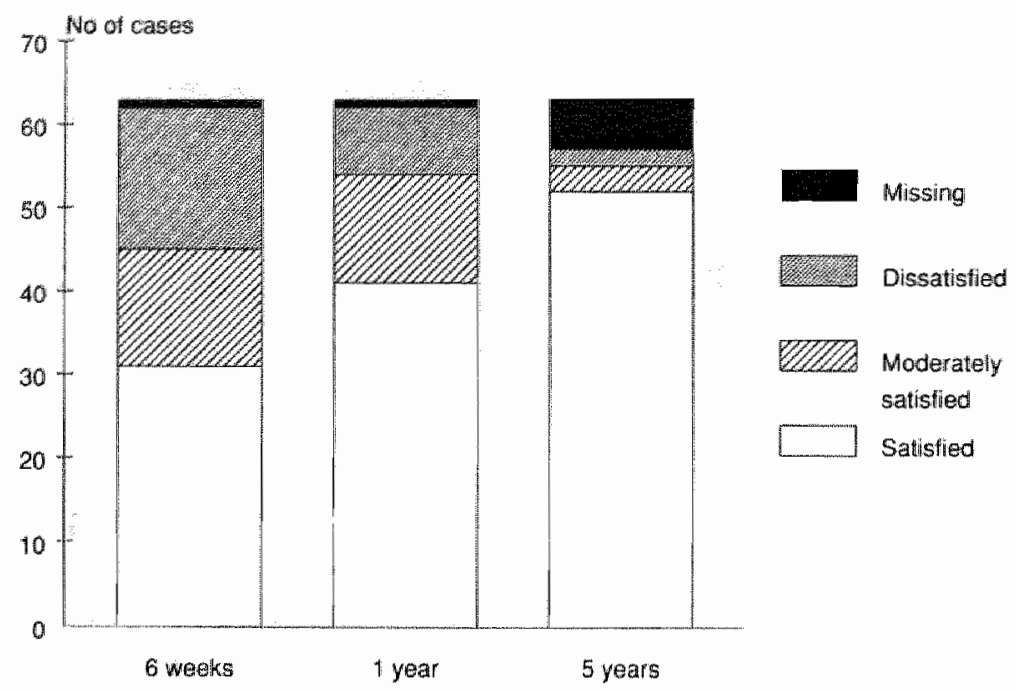

Figure 5-6.

Follow-up

Patients' assessments of the operative results at 6 weeks, at 1 year and at 5 years.

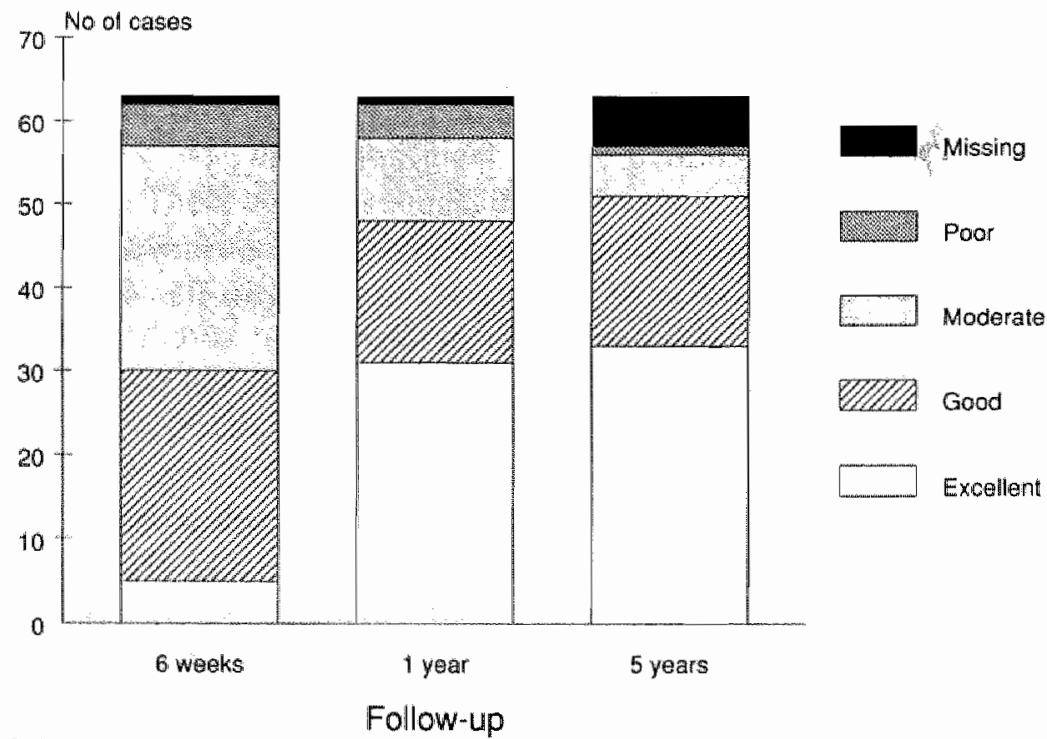

Figure 5-7.

Follow-up

Overall results of the operative treatment at 6 weeks, at 1 year and at 5 years. 
had only mild pain. All of these seven patients were satisfied with the results of treatment and in the overall result rating two had an excellent result and five a good result. Nonetheless, all said that they thought that their tennis elbow would recur if they returned to their original work.

Table 5-4.

Patients" ability to work before operation and at $\Downarrow$ year and 5 years after operation

Follow-up

Social status

0 weeks

1 year $\quad 5$ years

Same profession (Sick leave) (ziektewet)

Different profession, because of elbow

$44(32)$

$33(5)$

26

Different profession, not because of elbow

0

0

2

Disablement benefit (WAO) because of elbow

0

5

$\begin{array}{lll}0 & 5 & 7\end{array}$

Disablement benefit (WAO) not because of elbow

5

7

Not applicable

13

5

4

Retired

1

13

11

1

1

2

Missing

0

1

6

Number at follow-up

63

62

57

\subsubsection{Prognostic factors}

Several preoperative findings were correlated with the postoperative outcome: onset of complaints, professional activities, subjective loss of grip strength, severity of pain, limitation of extension, tenderness, pain provoked by resisted dorsiflexion of the wrist or extension of the third finger. We used the patient's assessment and the overall result rating as outcome parameters. However, none of these preoperative findings showed a statistically significant $(\mathrm{p}<0.05)$ relation with the results of operative treatment.

Furthermore, the age of the patient, the number of corticosteroid injections received, and the number of physiotherapeutical treatments showed no relation to the outcome of surgical treatment. Therefore in this series no significant relation between these variables and the outcome of treatment was demonstrated. 
Tallat $5 \cdot 5$

Numbers of corticosteroid injections received wersus overall result rating

No of injections

\begin{tabular}{lrl} 
Overall result rating & $\leq 3$ & $\geq 4$ \\
\hline Excellent & 23 & 8 \\
Good & 10 & 6 \\
Moderate & 5 & 6 \\
Poor & 3 & 1 \\
\hline
\end{tabular}

Local corticosteroid injections have been known to result in ruptures of tendons in some patients (Krahl and Plaue 1971). Some authors therefore warn against the use of corticosteroid injection in over-use disorders (Krahl and Langhoff 1971, Hermans 1977). A large number of corticosteroid injections at the lateral epicondyle may result in a weakening of the extensor origin and a poor outcome of surgical treatment. Table 5-5 shows the relation between the outcome of treatment (overall result rating) and the number of corticosteroid injections received. This table suggests that patients who received less than four corticosteroid injections obtained a better operative result than patients who got four of more injections. However, the difference between these two groups was non-significant.

\subsection{DISCUSSION}

In our patients the short-term results of operation were less impressive than those described by some authors (Hohmann 1933, Gardner 1970, Kammer and Bollinger 1981). Six weeks after operation, only 7 of the 62 patients were free of symptoms and only 8 of the 44 patients who had had a job had returned to work. For most of our patients 6 to 12 weeks were required for adequate, though not necessarily complete, recovery. The patients continued to improve during the first year after their operation, so that at one year 25 of them were completely free of symptoms (40\%). During the next four years of follow-up this number increased to $36(63 \%)$. This means that a considerable number of patients showed persisting lateral elbow pain notwithstanding the operation. Some other authors have observed the same phenomenon (Paar et al. 1978, O'Neill et al. 1980, Nollen 1981, Yerger 1985). Many symptomatic patients, however, had only minor symptoms, mainly slight pain after strenuous use of the forearm 
extensors. This improvement was documented by the grip strength measurements. These increased significantly after operation and the mean values for these grips were only slightly lower than those of the control (contralateral) arm at the one-year follow-up. The persistence of this slight pain is the reason why some authors have classified complete absence of symptoms as an excellent result and any minor symptoms as a good result (Nirschl 1979, Meine and Eicher 1981, Baumgard and Schwartz 1982, Litt and Albassir 1983). Using this classification, 48 per cent of our patients had an excellent or good result six weeks after operation. 77 per cent of patients had an excellent or good result one year after operation. Five years after operation, 90 per cent of the patients had an excellent or good result. One year postoperatively, 66 per cent of patients were satisfied with the results of operation, a figure which had risen to 91 per cent five years post-operatively.

It is difficult to compare our results to those in other studies as already noted, because only retrospective studies have been found in the literature, all of which showed serious limitations such as lack of information about the patients studied, about the techniques of assessment, about the numbers of patients followed up and about the criteria used for assessment (Bosworth 1955 and 1966, Friedlander 1967, Gardner 1970, Boyd and Mcleod 1973, Coonrad and Hooper 1973, Bowden 1978, Kaiser et al. 1982). In some studies the results of operation are presented without distinguishing between the various operative techniques used (Nirschl and Pettrone 1979) or between the treatment for medial and lateral epicondylitis (Coonrad and Hooper 1973, Kammer and Bollinger 1981, Meine and Eicher 1981, Baumgard and Schwarz 1982).

A summary of the results of various studies of operative results was presented in table 1-4. From this table we have selected those procedures which were similar to the type of operation we have performed (Table 5-6). The assessment of the results of these studies is based on the criteria described in table 1-3.

In table 5-6 excellent and good results were combined into one item. Some of the published articles do not specify the numbers of patients seen at follow-up, and others do not provide data about the size of the original group of patients operated on.

With the exception of the studies of Paar et al. (1978) and of Nollen (1981), most studies showed good results in more than 80 per cent of cases. In this respect, therefore, the results in our study are in agreement with those in most other series. No recurrences of symptoms were seen in our group of operated patients, suggesting that the results of this operation is enduring, at least for the five years covered by this study. 
Talble 5-6.

Resuts of the lateral release operation for tennis elbow in the literature compared with data from this sudy

\begin{tabular}{|c|c|c|c|c|c|c|c|c|}
\hline \multirow[b]{2}{*}{ Author } & \multirow[b]{2}{*}{ Year } & \multirow[b]{2}{*}{$\mathrm{N}-\mathrm{op}$} & \multirow[b]{2}{*}{ N-fu } & \multicolumn{3}{|c|}{ Results in percentages } & \multicolumn{2}{|c|}{ Follow-up } \\
\hline & & & & Good & Fair & Poor & Me & $\mathrm{n}(\min , \mathrm{max})$ \\
\hline Wiesner & 1952 & 10 & 10 & 100 & 0 & 0 & 一 & \\
\hline Spencer & 1953 & - & 49 & 95 & 0 & 5 & 13 & $(-,-120)$ \\
\hline Thormer & 1957 & - & 76 & 99 & 1 & 0 & - & $(24,96)$ \\
\hline Posch & 1978 & 67 & 35 & 88 & 5 & 7 & 96 & $(-,-)$, \\
\hline Paar & 1978 & - & 30 & 43 & 0 & 57 & 26 & $(04,48)$ \\
\hline Kammer & 1981 & 100 & 100 & 90 & 0 & 10 & 18 & $(03,84)$ \\
\hline Nollen & 1981 & 206 & 168 & 54 & 21 & 25 & 48 & $(-,-)$ \\
\hline Bengert & 1982 & 79 & 63 & 94 & 3 & 3 & - & \\
\hline Schimer & 1982 & 57 & 33 & 73 & 0 & 27 & - & $(03,108)$ \\
\hline Baumgard ${ }^{\#}$ & 1982 & 37 & 37 & 92 & 0 & 8 & 35 & $(14,81)$ \\
\hline Saillant & 1983 & 123 & 123 & 91 & 7 & 2 & 9 & $(-,-)$, \\
\hline Yerger & 1985 & 149 & 109 & 94 & 2 & 4 & - & $(12,132)$ \\
\hline Calvert & 1985 & 42 & 37 & 89 & 0 & 11 & 74 & $(-,-$, \\
\hline Waldis & 1989 & 101 & 63 & 80 & 5 & 15 & 20 & $(02,108)$ \\
\hline This study & 1992 & 63 & 57 & 91 & 5 & 4 & 58 & $(50,65)$ \\
\hline
\end{tabular}

$\mathrm{N}$-op: Number of elbows operated ( - indicates no numbers given by author); $\mathrm{N}$-fu: Number of elbows at follow-up ( - indicates no numbers given by author); \#: Release performed percutaneously; Follow-up: Mean follow-up and range (minimum and maximum) in months after operation ( - indicates absence of data).

At the five year follow-up, 7 patients (12\%) were on disablement benefit (WAO) because of persisting elbow complaints. We did not classify these patients as failures of treatment since there are many reasons for granting disablement benefits in the Netherlands, and of these medical reasons may not necessarily be the most important. Age, education level and unemployment may also influence the granting of such benefits. Based on our findings (two patients receiving benefits did not have any pain, and the other five sometimes had slight pain after forceful activities of their arms) we decided to ignore the patients' work status and to classify them on the basis of their complaints. 
We did not find any variables which were related to good or poor results. In the series described by Nollen (1981) and by Saillant et al. (1989) operative treatment was less successful for tennis elbow caused by occupational activities. However, their findings differed as to the effect of age on the outcome of treatment. Nollen also found that the operation was less successful in patients who had not responded to any conservative treatment, not even temporarily. Saillant et al. also reported that patients with a tennis elbow which had persisted for more than 2 years did not well after surgical treatment. Unfortunately, in both of these reports the statistical tests are not described and it may be that the conclusions were not tested statistically.

Theoretically, multiple corticosteroid injections may have a deleterious effect on the collagen tissue in the common extensor origin. No differences, however, were found between patients with more than 3 corticosteroid injections and those with 3 of these injections or less.

The number of complications encountered in our study was rather limited. Except for one infection, temporary limitation of extension was the only complication met. No patient had a persistent loss of extension. These figures are comparable to those in other studies.

At operation, three partial ruptures and one cyst of the extensor origin were noticed. Coonrad and Hooper (1973) and Nirschl and Pettrone (1979) described a much higher frequency of ruptures in the patients they operated upon. Nirschl and Pettrone (1979) also stated that the lesion is located in the origin of the extensor carpi radialis brevis muscle, and that it should be repaired. With the transverse division of the extensor origin that we used, only superficial ruptures of the origin could be seen. Therefore, we may not have found deep ruptures of the regular dense connective tissue of the origin.

The difficulties of interpreting the histology of the specimens were already discussed in Chapter 1. No histological changes that might be characteristic of tennis elbow have been clearly defined. Biopsies can only be obtained at operations on chronic lesions (Chard and Hazleman 1989). Moreover, the histological features of normal structures near the lateral epicondyle and of the changes associated with aging are not very well understood (Uhthoff and Sarkar 1980). However, in our series in agreement with the findings described in the literature, we found increased vascularity and round cell infiltration in the extensor origin. These findings would seem to support the common belief that the most frequent cause of tennis elbow is a lesion in the common extensor origin. Our histological findings can only be interpreted as providing some support for the 'rupture theory'. Definitive evidence remains to be found. 
Some authors, such as Kamien (1990), recommend a very conservative approach to surgical treatment because they believe that many patients subjected to early surgical intervention will recover without operation. Our patients were operated on because their pain had persisted for more than 6 months, was interfering with their occupations and with their activities during daily living and had not been alleviated, or only inadequately alleviated, by conservative treatment. In view of the rather favourable results of operative treatment in our series we do not think it is justified to deny these patients operative treatment. Our study did not address the question of how long conservative treatment should be continued or what factors should determine the indications for surgical treatment. The severity of pain, the cause of the complaints, the amount of impairment at work and in daily life as well as the patience of the patient may influence the decision relative to operative treatment.

Comparison of the results after the many operative procedures described in the literature does not indicate that any one technique is superior to others. Most studies report a success rate of over 80 percent. If, therefore, all operations are equally effective, a technique with a low morbidity should be selected. It was for this reason that we opted for the lateral extensor release described by Hohmann (1933), Spencer and Herndon (1953), Posch et al. (1978) and Calvert et al. (1985). Like these authors, we have found that the results after this procedure are gratifying while the complication rate is low. In addition, this operation used can be performed under local anaesthesia as an out-patient procedure. In our patients, intra-articular inspection did not reveal any relevant pathological changes. Therefore, it does not seem logical to perform routine arthrotomy, a procedure that could increase postoperative morbidity. Currently, we regard lateral release of the extensor origin as the preferred technique, with which other operations should be compared.

From a theoretical point of view, operative techniques that include excision and repair of a rupture or scar tissue, as described by Coonrad and Hooper (1973), Nirschl and Pettrone (1979) and Froimson (1982) deserve more study. However, so far, none of these procedures has been shown to have advantages over traditional techniques. Baumgard and Schwartz (1982) and Yerger and Turner (1985) described good results after percutaneous lateral release. This may be a good alternative to an open procedure such as the one we have performed. A comparative study would be desirable. 


\section{5 .}

CONCLUSIONS

From this study it may be concluded that:

- a considerable number of patients continued to have some lateral elbow pain notwithstanding the operation.

- $40 \%$ of the patients were completely symptom-free one year after surgery and $63 \%$ of the patients were so 5 years after surgery.

- $77 \%$ of patients had an excellent or good result one year after operation. Five years after operation, 90 per cent of the patients had an excellent or good result.

- at the review after one year $66 \%$ of patients were satisfied with the results of operation, a figure which rose to $91 \%$ of patients 5 years postoperatively.

- no predictors of the results of treatment were found.

- the number of complications of the operative treatment was rather low.

- lateral release of the extensor origin may be regarded as the surgical management of choice and should be the preferred technique with which the results of other tennis elbow operations should be compared. 


\section{CHAPTER}

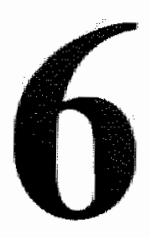

\section{The Lateral Cubital \\ Force Transmission \\ System}

A study of the possibilities of localizing a tennis elbow 'lesion' within the Lateral Force Transmission System of the elbow 


\title{
THe LATERAL CUBitAl ForCE TRANSMISSION SYSTEM
}

\begin{abstract}
A study of the possibilities of localizing a tennis elbow 'lesion' within the Lateral Force Transmission System of the elbow
\end{abstract}

\subsection{INTRODUCTION}

Many causes of tennis elbow have been suggested, but the most generally supported hypothesis is that (micro)ruptures in the regular dense connective tissue at the lateral epicondyle are the main cause of the complaints.

In current practice, tennis elbow is diagnosed on the basis of local tenderness at the origin of the forearm extensors and pain provoked by resisted dorsiflexion of the wrist. This limited physical examination may not provide sufficient data about the precise location of lesions in the lateral elbow region. If it were possible to localize lesions in tennis elbow complaints more accurately, this might lead to more selective and restricted conservative and operative treatment.

Localization of ruptures in the dense connective tissue should be based on physical examination based on detailed anatomical knowledge. Few papers about tennis elbow have concentrated on the anatomy of the lateral elbow region and most authors have used the rather limited description in most current textbooks of anatomy. Recently, several authors have published new data about the anatomical features of the lateral elbow region (Heyse-Moore 1984, Briggs and Elliott 1985, Van Mameren and Drukker 1984). This has led to a description of the way in which tensile forces generated by the muscle fibres of the forearm muscles are transmitted to the lateral part of the humerus (Van Marneren and 
Drukker 1984). This is accomplished by a system we have called Lateral Cubital Force Transmission System (LCFTS) (Chapter 2). With the exception of the extensor carpi radialis longus, all contractile elements (muscular fibres) of the extensors of the wrist and hand are attached to the regular dense connective tissue of this LCFTS. The part of the LCFTS which conducts the tensile stresses depends upon the muscle which is activated. We have postulated that (micro)ruptures would provoke most pain when tensile stresses are conducted through the part of the LCFTS in which the rupture is located.

This led us to perform a study in order to investigate whether the suspected pathology in tennis elbow could be localized using a diagnostic system based on the concept of the Lateral Cubital Force Transmission System (LCFTS) of the elbow. This diagnostic system was based on the assumption that the combination of several resisted movements and accurate palpation of the lateral elbow region would lead to localization of one or more 'lesions' in the LCFTS in patients with a tennis elbow. It was further hypothesized that classification of patients using such a diagnostic system would relate to different outcome of treatment for these patients.

\subsection{PATIENTS AND METHODS}

The study was conducted simultaneously with the studies of the results of conservative treatment (Chapter 4) and of the results of operative treatment (Chapter 5). For the present study, patients seen at the "elbow clinic' for the other studies were also examined by a physician from the Department of Anatomy of the University of Limburg (head: Prof. dr. J. Drukker). 101 patients were examined before the start of treatment and 86 patients were re-examined one year later. Not all patients seen at the 'elbow clinic' were involved in the present study. This was due to the fact that not all sessions at the out-patient clinic could be attended by the anatomist. However, this was a random occurrence without any selection of patients.

The anatomist was blinded for the treatment of the patients both at the start of the treatment and at the one year follow-up (with the exception of the operated patients). Of the 101 patients seen before treatment was started 78 were treated purely conservatively. The remaining 23 patients were operated upon.

The physical examination used in this study consisted of two parts:

1- Meticulous palpation of the lateral elbow region. Each of the areas listed in table 6-1 was palpated and was scored as painful or non-painful. 
Physical cxamination of the lateral elbow region

Palpation: (with the elbow in 90 degrees of flexion and the forearm in mid-pronation)

- Supracondylar ridge

- Proximial lateral epicondyle

- Top of lateral epicondyle

- Ventral of lateral epicondyle

- Distal of lateral epicondyle

- Medio-ventral of radial head

- Latero-dorsal of radial head

Muscle bellies of:

- Extensor carpi radialis longus (ECRL)

- Extensor carpi radialis brevis (ECRB)

Extensor digitorum (ED)

- Extensor digiti minimí (EDM)

- Extensor carpi ulnaris (ECU)

- Anconeus

- Triceps

- Supinator

- Pronator teres

Septum:

- between ECRB and ED muscle

- between ED and ECU muscle

Resisted movements: (with the elbow in 90 degrees of flexion and the forearm in midpronation)

- Dorso-radial extension of the wrist

- Extension of digit $\mathrm{V}$

- Extension of digit IV

- Extension of digit III

- Extension of digit II

- Extension of digit I

- Pronation of the forearm resisted at the wrist

- Pronation of the forearm resisted at the hand

- Supination of the forearm resisted at the hand

- Supination of the forearm resisted at the wrist

- Fllexion of the elbaw resisted at the wrist

- Flexion of the elbow resisted at the hand

- Extension of the elbow resisted at the wrist

- Extension of the elbow resisted at the hand 
2- Resisted movements of elbow, forearm, wrist and hand, in order to put selective strain on different parts of the LCFTS. Each of the resisted movements was scored as painful or non-painful.

Table 6-1 presents a survey of the examination. A detailed description of the corresponding anatomical relations between regular dense connective tissue and muscular fibres was given in Chapter 2. The physical examination led to one of three conclusions:

A- 'Lesion' in the Lateral Cubital Force Transmission System (LCFTS) of the elbow can be localized in detail.

In these patients four sites in the LCFTS were distinguished:

I - Septum between Extensor Carpi Radialis Brevis muscle (ECRB) and Extensor Digitorum muscle (ED)

II - Extensor Digitorum muscle (ED)

III - Septum between the extensor digitorum muscle (ED) and the extensor carpi ulnaris muscle (ECU)

IV - Anconeus muscle

B- 'Lesion' in the Lateral Cubital Force Transmission System (LCFTS) cannot be localized in detail because of contradictory findings.

C- 'Lesion' cannot be localized in the Lateral Cubital Force Transmission System (LCFTS). Other causes of tennis elbow complaints should be considered.

As parameters for the outcome of treatment we used some of the parameters described in chapter 4 (Table 4-2): severity of pain, subjective loss of grip strength, resisted dorsiflexion of the wrist, resisted dorsiflexion of digit 3, local tenderness, patient"s assessment and overall result rating.

\section{Statistics}

All data are given as numbers of patients. The McNemar-test was used to test the differences in findings at palpation at the start of treatment and one year postoperatively. The same test was used for the analysis of the difference in pain provoked by resisted movements. The $\mathrm{X}^{2}$-test for linear trend was used to determine the relationship between the conclusion in the present study and the outcome of treatment (Cochran 1954). The Fisher-exact test was used to analyze 
the conclusions of the physical examination in order to find possible correlations with the end result of treatment (Overall result rating). $P$-values $<0.05$ were considered to be statistically significant.

\subsection{RESULTS}

Table 6-2 lists the findings of palpation at the start of treatment and at the one year follow-up. Many patients had more than one location which was painful at palpation. The most frequently painful regions were:

- ventral of the lateral epicondyle;

- medio-ventral of the radial head;

- muscle belly of ECRB muscle or ED muscle;

- septum between ECRB and ED muscles.

At the one year follow-up, some areas were painful in fewer patients. This decrease in the frequency of painful areas was most pronounced:

- at the top of the epicondyle

- ventral of the lateral epicondyle

- over the muscle bellies of the ECRB and ED muscles.

Palpation medio-ventral of the radial head remained painful in a large number of patients.

Table 6-3 lists the findings for resisted movements of elbow, wrist, hand and fingers before the start of treatment and at one year follow-up.

Resisted dorsiflexion of the wrist at full extension of the ellbow was painful in all patients. However, resisted dorso-radial extension of the wrist with the elbow in a position of 90 degrees of flexion was painful in 65 of 86 patients (76\%). At the one-year follow-up, fewer patients experienced pain provoked by resisted movements. This decrease was most pronounced for the dorso-radial abduction and the extension of the digits 3 and 4 . The pain provoked by resisted pronation or supination of the forearm with resistance given by holding the wrist was less painful than the same resisted movements with resistance given by holding the hand. The same observation was made for flexion and extension of the elbow.

The conclusions of the physical examination at 0 and 52 weeks are listed in table 6-4. 
Table 6-2.

Findings at palpation at 0 weeks and at 52 week in 86 patients with a tennis elbow

\begin{tabular}{|c|c|c|c|}
\hline Tendemess & $\begin{array}{l}\text { Pre-treatment } \\
0 \text { weeks }\end{array}$ & $\begin{array}{l}\text { Post-itreatment } \\
52 \text { weeks }\end{array}$ & p-values \\
\hline - Supracondylar ridge & 3 & 11 & $* *$ \\
\hline - Proximal lateral epicondyle & 10 & 6 & $*$ \\
\hline - Top of lateral epicondyle & 24 & 5 & $* x^{2}$ \\
\hline - Ventral of lateral epicondyle & 55 & 20 & $* * *$ \\
\hline - Distal of lateral epicondyle & 34 & 16 & 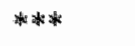 \\
\hline - Medio-ventral of radial head & 60 & 50 & $*$ \\
\hline - Latero-dorsal of radial head & 18 & 16 & $*$ \\
\hline \multicolumn{4}{|l|}{ Muscle bellies of } \\
\hline - Extensor carpi radialis longus & 4 & 4 & $*$ \\
\hline - Extensor carpi radialis brevis & 54 & 21 & ***a* \\
\hline - Extensor digitorum & 58 & 16 & $* * *$ \\
\hline - Extensor digiti minimi & 4 & 3 & $*$ \\
\hline - Extensor carpi ulnaris & 16 & 5 & $* *$ \\
\hline - Anconeus & 6 & 5 & $*$ \\
\hline - Triceps & 5 & 5 & * \\
\hline - Supinator & 4 & 12 & ** \\
\hline - Pronator & 0 & 3 & $*$ \\
\hline \multicolumn{4}{|l|}{ Septum } \\
\hline \multicolumn{4}{|c|}{ - between extensor carpi radialis brevis } \\
\hline $\begin{array}{l}\text { and extensor digitorum muscle } \\
\text { - between extensor digitorum an }\end{array}$ & 52 & 35 & $* *$ \\
\hline extensor carpi ulnaris muscle & 14 & 4 & $* *$ \\
\hline
\end{tabular}

$*$ Non-significant; **<0.05;***<0.01

At 0 weeks, it was concluded that 73 of the 86 patients had a 'lesion' in the LCFTS, which could be localized in detail in 68 of them. In 5 patients the pain pattern was too diffuse to allow a precise localization of the 'lesion'. In 13 patients (15\%) no 'lesion' in the LCFTS was identified. One year later, a 'lesion' in the LCFTS was identified in 35 of 86 patients ( $41 \%$ ). There was uncertainty about the exact localization of the 'lesion' in 6 patients. No "lesion' in the LCFTS was identified in 45 patients. In 32 of these 45 patients no pain could be provoked in the elbow region. The remaining 13 patients had provokable pain in the elbow region, but it was concluded that this pain was not located in the 
Table 6.3.

Pin att resisted movements at 0 weeks and 52 weeks after start of treatment $(N=86)$

\begin{tabular}{|c|c|c|c|}
\hline \multirow[b]{2}{*}{ Resisted movement } & \multicolumn{3}{|l|}{ Painful } \\
\hline & 0 weeks & 52 weeks & p-value \\
\hline Dorso radial extension of the wrist & 65 & $\mathbb{1 9}$ & $* * *$ \\
\hline Extension of digit $V$ & 9 & 2 & *** \\
\hline Extension of digit IV & 30 & 8 & $* * *$ \\
\hline Extension of digit III & 52 & 20 & $* * *$ \\
\hline Extension of digit II & 16 & 5 & $* *$ \\
\hline Extension of digit I & 1 & 1 & * \\
\hline Pronation of the forearm resisted at the wrist & 25 & 11 & $* * *$ \\
\hline Pronation of the forearm resisted at the hand & 55 & 17 & $* * *$ \\
\hline Supination of the forearm resisted at the wrist & 29 & 14 & $* * *$ \\
\hline Supination of the forearm resisted at the hand & 59 & 22 & $* * *$ \\
\hline Flexion of the elbow resisted at the wrist & 8 & 8 & * \\
\hline Flexion of the elbow resisted at the hand & 42 & 16 & $* * *$ \\
\hline Extension of the elbow resisted at the wrist & 12 & 6 & * \\
\hline Extension of the elbow resisted at the hand & 16 & 6 & $* * *$ \\
\hline
\end{tabular}

* Non-significant; ** $<0.05 ; * * *<0.01$

\section{Table 6-4.}

Conclusions of physical examination at 0 weeks and 52 weeks after start of treatment in patients with a tennis elbow $(\mathrm{N}=86)$

\section{2 weeks}

\section{LCFTS Non-localizablle No LCFTS (pain still}

0 weeks

Total lesion LCFTS lesion lesion prowokable)
LCFTS lesion

Non-localizable

\begin{tabular}{lrrrrr} 
LCFTS lesion & 5 & 1 & 1 & 3 & $(2)$ \\
No LCFTS lesion & 13 & 4 & 1 & 8 & $(2)$ \\
Total & 86 & 35 & 6 & 45 & (13) \\
\hline
\end{tabular}


LCFTS. Because most patients were concluded to have a LCFTS 'lesion', the 2 other groups were rather small. The subdivision in table 6-4 did not show significant differences between the findings at 0 and at 52 weeks. In 68 patients a 'Iesion' in the Lateral Cubital Force Transmission System (LCFTS) could be localized in detail. The precise sites of the 'lesion's' in the LCFTS are listed in table 6-5. In several patients more than one site was identified. The regular dense connective tissue septum from which the extensor carpi radialis brevis muscle and the extensor digitorum muscle originate was most frequently identified as the site of the 'lesion'.

Table 6-5.

Site of the suspected 'lesion" in the Lateral Cubital Force Transmission System at 0 weeks and 52 weeks after start of treatment in patients with a tennis elbow $(\mathrm{N}=86)$

Site

0 weeks

52 weeks

Septum between ECRB muscle and ED muscle

49

16

Muscle belly of the extensor digitorum

52

27

Septum between ED muscle and ECU muscle

15

Muscle belly of the anconeus

13

The conclusions of table 6-4 led to distinguish between 2 groups of patients; those with 'lesion' in the LCFTS (lesion or possible lesion) and without a 'lesion' in the LCFTS (no lesion). Based on the assumption that the first group would have better results of treatment than the second one, the two groups were compared on several outcome parameters of treatment at 52 weeks. Patients classified as having a 'lesion' in the LCFTS had better results on all parameters listed in table 6-6. However, the differences between the 2 groups ("with' and 'without' a 'lesion' in the LCFTS') were non-significant. The same analysis was done for those patients in whom a 'lesion' was identified which could be localized in detail. These patients formed group I (lesion), while the remaining patients formed group II (possible lesion or no lesion). Table 6-7 shows the relation between the end result of treatment (Overall result rating) and these 2 groups.

Group I seemed to have better results of treatment than group II. However, statistical testing (Fisher exact test: $p=0.24$ ) showed no significant difference between groups I and II. 
Talble $6-6$

Differences in the outcome of treatment between patients with $(n=85)$ and without $(n=16)$ a 'lesion" in the Lateral Cubital Force Transmission System and the outcome of treatment at 52 weeks

Correlation coefficient

$\begin{array}{llr}\text { I. Severity of pain } & 0.084 \\ \text { II Subjective loss of grip strength } & 0.030 \\ \text { III Resisted dorsiflexion of the wrist } & 0.001 \\ \text { IV Resisted dorsiflexion of digit 3 } & 0.177 \\ \text { IV Local tenderness } & 0.140 \\ \text { V Patient's assessment } & -0.175 \\ \text { VI Overall result rating } & 0.150\end{array}$

All coefficients are non-significant

Table 6-7.

Outcome of treatment at one year follow-up (Overall result rating) for patients with a "lesion" in the LCFTS which could be localized (group I) and the remaining patients (group II)

\begin{tabular}{llll}
\hline Results & Group I & Group II & Total \\
\hline Excellent / Good & 53 & 14 & 67 \\
Moderate / Poor & 24 & 10 & 34 \\
Total & 77 & 24 & 101 \\
\hline
\end{tabular}

\subsection{DISCUSSION}

The concept of the Lateral Cubital Force Transmission System (LCFTS) was developed on the basis of several anatomical studies. Morphologically, this system has been identified at dissection, MRI and CT-scanning (Van Mameren and Drukker 1984, Van Mameren et al. 1985).

The clinical significance of the LCFTS for the pathogenesis and treatment of tennis elbow has, however, not yet been studied. Our study was based on the assumption that the combination of several resisted movements and accurate palpation of the lateral elbow region would lead to localization of one of more 
lesions in the LCFTS. It was further hypothesized that a classification of patients using a diagnostic system based on the concept of the Lateral Cubital Force Transmission System (LCFTS) would relate to different outcomes of treatment for these patients.

At the one year follow-up, the supinator and the supracondylar ridge were the only sites with increased tenderness compared with the situation at 0 weeks. At 52 weeks, the tenderness medio-ventral and latero-dorsal of radial head was almost unchanged compared with the findings at 0 weeks. These regions are also painful at palpation in many persons without tennis ellbow complaints. This might explain why tenderness in these regions did not show improvement, while other regions did.

Dorsiflexion of the wrist at full extension of the elbow was painful in all patients: However, dorsoradial abduction of the wrist with the elbow in 90 degrees of flexion (and the elbow in mid-pronation) provoked pain in only 75 per cent of patients. The latter movement requires the use of the extensor carpi radialis brevis and longus muscle. Dorsiflexion of the wrist at full extension of the elbow, involves the extensor digitorum and extensor carpi ulnaris as well. In addition, the flexion angle of the elbow may also be important, but no data are available about the changes in transmission of the forces in the LCFTS in relation to the degree of elbow flexion. It was shown that the site of resistance in the resisted movements was important. The wrist is stabilized by muscles originating from the LCFTS, which means that if resistance is given by holding the hand, the wrist must be stabilized. The muscle activity required for stabilizing the wrist might provoke pain in patients with tennis elbow.

The conclusions of the physical examination cannot be verified by means of radiographs or other presently available techniques. Therefore, we have tried to substantiate these conclusions by correlating them with the one year results of treatment. In fact, we analyzed the prognostic value of the detailed physical examination of the LCFTS, which resulted in the distinguishing of 2 groups of patients. A diagnosis of 'lesion' in the LCFTS seemed to predict in a better outcome of treatment at 52 weeks, but this was not statistically significant. However, in our study the group without such a 'lesion' included only 15 per cent of the patients. In such a small group onlly major differences would have been statically significant.

We used rather restrictive inclusion and exclusion criteria in sellecting patients for our study. For these selected tennis elbow patients, we were unable to prove that a more detailed physical examination based on the concept of the LCFTS did have advantages over the traditional orthopaedic examination with respect 
to diagnosis and treatment of tennis elbow. However, if less restrictive criteria for diagnosing tennis elbow had been used, as is common in regular general practice and in orthopaedic out-patient clinics, it is not unlikely that the more detailed physical examination would have identified groups with a good and a poor response to treatment.

It was especially the resisted movements resulting in tensile stresses conducted by the LCFTS which provoked pain. In many patients, it was particularly the resisted movements of the extensor carpi radialis brevis muscle and the extensor of the middle finger which induced pain. The involvement of these two muscles can be explained from the origin of the muscles as described in Chapter 2. Both muscles originate from their common septum. The involvement of this region is in agreement with the reports by Coonrad and Hooper (1973) and Nirschl (1979), who described ruptures and scar tissue in the origin of the extensor carpi radialis brevis muscle.

The findings of this study illustrate that the concept of the Lateral Cubital Force Transmission System can explain several aspects of tennis elbow which could not be explained very well before. This concept explains why resisted extension of the middle finger, the second digit and resisted dorsiflexion of the wrist produce pain in many patients with tennis elbow. The relation between the localization of tenderness and pain produced by resisted movement can also be explained very well using the concept of the LCFTS of the elbow. The next step should be histological confirmation of the suggested 'lesions'. The regular dense connective tissue of the LCFTS of the elbow can be recognized on MRI-scans. Using MRI-scans in patients with a tennis elbow might show these 'lesions' in the LCFTS and might improve our insight into the pathogenesis of tennis elbow. Further elaboration of the concept of the LCFTS might lead to a more selective treatment of tennis elbow, leading to better results of treatment.

\subsection{CONCLUSIONS}

From this study it may be concluded that:

- the extensor carpi radialis brevis, the intermuscular septum between the extensor carpi radialis brevis and the extensor digitorum, as well as the extensor digitorum (especially the musculotendinous unit of digit 3 ) were identified as the most frequent sites of pain in tennis elbow. 
- one year after the start of treatment, the areas near the epicondyle were less often painful than the other areas.

- an accurate physical examination based on the concept of the Lateral Cubital Force Transmission System seemed to select a group of patients with less favourable results of treatment, but this difference was statistically non-significant. 



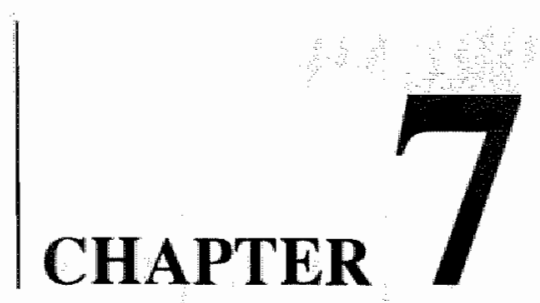

\section{Radial tunnel syndrome}

\section{An investigation of compression neuropathy as a possible cause}

This chapter has been published in the Journal of Bone and Joint Surgery (Am), 1991;73A:539-44. 


\section{An investigation of compression neuropathy as a possible cause}

\section{1.}

\section{INTRODUCTION}

The concept of radial tunnel syndrome in which the radial nerve and its branches are intermittently or constantly compressed was introduced by Roles and Maudsley in 1972. They suggested that, depending on the severity, duration, and site of compression of the radial nerve, there could be a wide spectrum of clinical syndromes, ranging from socalled tennis elbow to irreversible paralysis of the muscles supplied by the radial nerve. They treated 36 patients who had resistant tennis elbow by decompression of the radial nerve and obtained improvement in 35 of them. Electromyographic studies were carried out in 10 patients and were considered to confirm compression of the radial nerve in 8 .

However, a well documented prospective study by Van Rossum et al. (1978) did not reveal any abnormalities in the electromyograms and nerve-conduction studies of 10 patients who had persistent tennis elbow. Information about the electromyographic findings in other studies of the radial tunnel syndrome has usually been deficient (Lister et al. 1979, Ritts et al. 1987, Werner 1979). In elaborate studies, Rosén and Werner found only slight abnormalities in the electromyographic and nerve-conduction studies in a minority of 28 patients; all 28 had tennis elbow that was resistant to non-operative treatment (Rosén and Werner 1980). Those authors suggested that the negative findings could be explained by the intermittence of the compression of the interosseous nerve. They noted that active supination of the forearm produced an increase in the 
conduction time of the posterior interosseous nerve across the supinator muscle more often in patients who had radial tunnel syndrome than in control subjects (Rosến and Werner 1980).

Therefore, we used the technique of Rosén and Werner to increase the sensitivity of our neurophysiological studies in patients who had a clinical diagnosis of a radial tunnel syndrome. In this chapter we describe the results of this study.

\subsection{PATIENTS AND METHODS}

\subsubsection{Patients}

From 1986 to 1987 , radial tunnel syndrome was clinically diagnosed in 16 patients at our hospital (Table 7-1). There were 11 man and 5 women, all of whom had long-standing pain in the lateral aspect of the elbow that was resistant to therapy. The ages of the patients ranged from 28 to 62 years (mean age 44 years). The mean duration of the symptoms was 84 weeks. Thirteen patients had pain in the right forearm and 3 in the left. Three patients (cases 3,6 and 7) had a previous operation (soft-tissue release of the elbow) for tennis elbow.

The clinical diagnosis of radial tunnel syndrome was based on the criteria of Werner (1979):

1- Pain in the proximal radial part of the forearm, which is aggravated by work but also present at rest. 2- Intense tenderness over the posterior interosseous nerve, especially where it passes under the proximal edge of the superficial supinator muscle, approximately 5 centimeters distal to the lateral epicondyle.

All 16 patients fulfilled both criteria. Active supination against resistance was painful in 11 patients. Tenderness during resisted dorsiflexion of the wrist was noted in all patients. Fifteen patients had pain on resisted extension of the middle finger. Resisted pronation was painful in 5 patients. Eight patients had aggravation of the pain during venostasis, elicited by use of a tourniquet on the arm. There was no neurological deficit in any patient. Ten patients were operated on with the procedure to be described. The other 6 patients were treated non-operatively. 


\begin{tabular}{|c|c|c|c|c|c|c|c|c|}
\hline \multirow[b]{2}{*}{$\mathrm{No}$} & \multirow[b]{2}{*}{ Sex } & \multirow[b]{2}{*}{ Age } & \multirow[b]{2}{*}{ Side } & \multirow[b]{2}{*}{ Profession } & \multirow{2}{*}{$\begin{array}{l}\text { Period of } \\
\text { complaints } \\
\text { (weeks) }\end{array}$} & \multirow{2}{*}{$\begin{array}{l}\text { Operation } \\
\text { Tennis } \\
\text { elbow }\end{array}$} & \multicolumn{2}{|c|}{$\begin{array}{l}\text { Decompression of } \\
\text { the Radial nerve }\end{array}$} \\
\hline & & & & & & & +1 & Resiult \\
\hline 1 & $\mathbf{M}$ & 62 & $\mathrm{R}$ & Restorer & 60 & No & No & - \\
\hline 2 & $\mathrm{M}$ & 62 & $\mathbf{R}$ & Unemployed & 70 & No & Yes & Fair \\
\hline 3 & $\mathbf{M}$ & 48 & $\mathbf{R}$ & Furniture maker & 150 & Yes & Yes & Fair \\
\hline 4 & $\mathrm{~F}$ & 45 & $\mathrm{~L}$ & Cleaning woman & 120 & No & Yes & Poor \\
\hline 5 & $\mathrm{M}$ & 46 & $\mathrm{R}$ & Bricklayer & 150 & No & Yes & Poor \\
\hline 6 & $\mathrm{M}$ & 35 & $\mathbf{R}$ & Motor mechanic & 50 & Yes & Yes & Poor \\
\hline 7 & $\mathrm{M}$ & 52 & L & Unemployed & 60 & Yes & No & - \\
\hline 8 & $\mathrm{~F}$ & 42 & $\mathrm{R}$ & Housewife & 70 & No: & Yes & Poor \\
\hline 9 & $F$ & 39 & $\mathrm{R}$ & Cleaning woman & 150 & No & No & - \\
\hline 10 & $\mathbf{M}$ & 43 & $\mathrm{R}$ & Unemployed & 10 & No & Yes & Good \\
\hline 11 & $\mathrm{M}$ & 28 & $\mathbf{R}$ & Mechanic & 36 & No & No & - \\
\hline 12 & $F$ & 46 & $\mathbf{R}$ & Housewife & 18 & No & No & - \\
\hline 13 & $\mathbf{M}$ & 31 & $\mathrm{R}$ & Truck driver & 35 & No & No & - \\
\hline 14 & $\mathbf{M}$ & 31 & $\mathbf{R}$ & Cleaning woman & 150 & No & Yes & Poor \\
\hline 15 & $\mathrm{M}$ & 54 & $\mathbb{R}$ & House-painter & 130 & No & Yes & Poior \\
\hline 16 & $\mathrm{~F}$ & 32 & $\mathbb{R}$ & Waitress & 80 & No & Yes & Fair \\
\hline
\end{tabular}

\subsubsection{Control group}

A control group consisting of 36 healthy volunteers who did not have a history of pain in the lateral aspect of the elbow, also was tested. There were 24 men and 12 women, and the ages ranged from 19 to 32 years (mean age 25 years).

\subsubsection{Measurements of force}

The subject was seated in front of a specially designed force-recording apparatus (Figure 7-1). The forearm was supported by a flat pillow for horizontal positioning. The elbow was placed in about 65 degrees of flexion and the arm was prevented from being pushed against the body. The subject exerted prolonged isometric pronation or supination against a vertical, fixed-position handle, which was mechanically connected to a strain-gauge force transducer. 


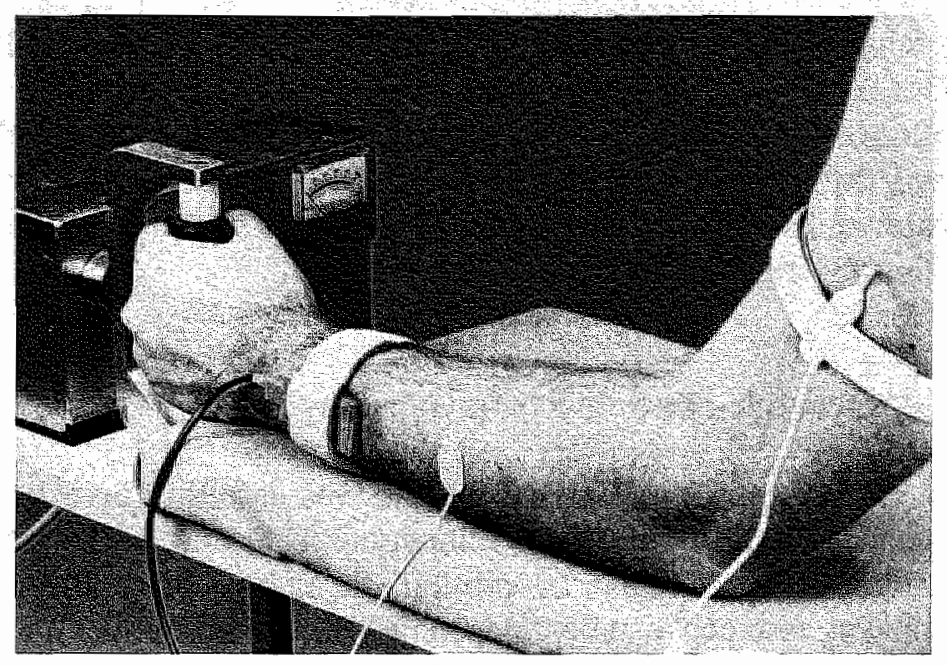

Figure 7-1.

Force recording apparatus, with (from distal to proximal) recording electrode, ground electrode, and stimulating electrode.

The strain-gauge was interfaced to a direct-current amplifier, the analog output of which was indicated by a voltmeter. Maximal pronation and supination forces were determined for both right and left arm. The subject was instructed to use only the arm, keeping the shoulders and the rest of the body relaxed and in a neutral position.

\subsubsection{Nerve-conduction studies}

Surface electrodes were used for stimulation and recording. The active recording electrode was placed over the extensor indicis proprius muscle, 7 centimeters proximal to the wrist, and the reference electrode was placed 4 centimeters farther distally. The radial nerve was stimulated with surface electrodes placed 10 centimeters proximal to the lateral epicondyle. The duration of the stimulus was 0.5 millisecond, with a current ranging from 50 to 78 milliamperes. The subject was asked to grip the handle, and motor latency was measured without active supination. Then the subject was asked to maintain 50 per cent of the maximum supination force for 60 seconds. The force was indicated by the voltmeter (Figure 7-1). The radial nerve was stimulated supramaximally 8 times, at intervals of 10 seconds; that is, the last stimulation occurred after ten seconds of relaxation. Motor latencies were measured to the onset of the compound muscle action potential. The amplitude of the compound muscle action potential 
had to remain maximum to ensure that all motor-nerve fibers were activated at each stimulus. This was often difficult to achieve because the co-contracting muscles of the arm tended to push the stimulating electrode away from the nerve. In these cases, the stimulating electrode was firmly pressed against the arm by the investigator and higher currents and longer durations of stimulus (as long as one millisecond) were applied.

\subsubsection{Concentric-needle electromyography}

In all patients, the extensor digitorum muscle was carefully investigated for signs of denervation (fibrillations) or reinnervation (increased percentage of polyphasic motor-unit potentials). In addition, the interference pattern on maximum volition was assessed visually. Depending on the differential diagnosis, a variable number of other muscles in the arm were investigated as well.

\subsubsection{Operative technique}

Ten patients were operated on. The operative exposure was through the anterior approach with use of an incision distal to the crease of the elbow and extending over the radial head (Roles and Maudsley 1972). The deep fascia was divided and the brachioradialis muscle was exposed and retracted radially. The muscles were inspected for ruptures or abnormal tissue, but no dissection other than for visualization of the radial nerve was carried out. The radial nerve was exposed and the veins running over it were transversely divided. The posterior interosseous nerve was followed distally and the fibrous edge of the supinator was divided longitudinally. A free fat transplant was placed over the nerve. Only the subcutaneous tissue and skin were sutured, and a soft compression dressing was applied.

\subsection{RESULTS}

\subsubsection{Measurements of force}

Because of the limited number of patients and the wide range of maximum supination forces, a statistical analysis of differences in force was not feasible (Table 7-2). Very low values of supination forces seemed to be due to less-than- 
Table 72 .

Maximum supination force, motor latency, and increase in latency during sixty seconds of supination (maximum, 50 per cent) in patients and control subjects

\section{Patients}

\section{Normal arm}

Left

Painful arm

Left Right

Control subjects

Left Right

24

24

Men (no.)

10

2

2

10

$3.0 \quad 7.4$

8.4

9.2

Mean

9.6

5.8

$7.6-15.0$

$4.0,7.6$

$3.0,3,0 \quad 4.2-15.0$

$6.4-11.2 \quad 7.0-11.6$

Motor latency (ms)

$\begin{array}{lllllll}\text { Mean } & 6.5 & 6.1 & 6.0 & 6.6 & 6.2 & 6.2 \\ \text { Range } & 5.8-7.7 & 5.6,6.5 & 5.4,6.6 & 6.2-7.6 & 5.6-7.3 & 5.8-6.8\end{array}$

Increase in latency (ms)

Mean

0.1

Range

$-0.1-0.4$

0.1

$0.0,0.2$

0.2

0.11

$0.0,0.3 \quad-0.1-0.4$

1

1

3

2.9

4.1

$3.8-4.7$

4.1

4.9

Range
$4.4-5.0$

4.2

5.2

$5.1-6.9$

$5.9 \quad 5.2$

5.0

5.7

4.9-6.5
$0.1 \quad 0.2$

$-0.1-0.4 \quad 0.0-0.4$

Motor latency (ms)

Mean

Range

Increase in latency (ms)

Mean

$\begin{array}{ll}0.2 & 0.0 \\ 0.0,0.3,0.4 & \end{array}$

0.0

0.4

0.1

0.1

Range

$$
0.0,0.3,0.4
$$

$0.0,0.2,1.0^{\text {覀 }}$

$0.0-0.3$

* This arm was the only one which had a major increase in latency. 
optimum cooperation by the patient because of pain. In these instances, the maximum pronation force was usually lower in the painful arm as well. Most maximum forces, however, were within the range of the control values.

\subsubsection{Nerve-conduction studies}

The range of latency values was small. There were no statistically significant differences in the motor latencies between painful and pain-free arms or between patients and control subjects (Table 7-2). During the 60 seconds of supination, there was a slight increase in the latencies in the control subjects, that never exceeded 0.4 millisecond. Only one patient (a woman) had a major increase in latency (to 1.0 millisecond) (Figure 7-2). The latency increase occurred even

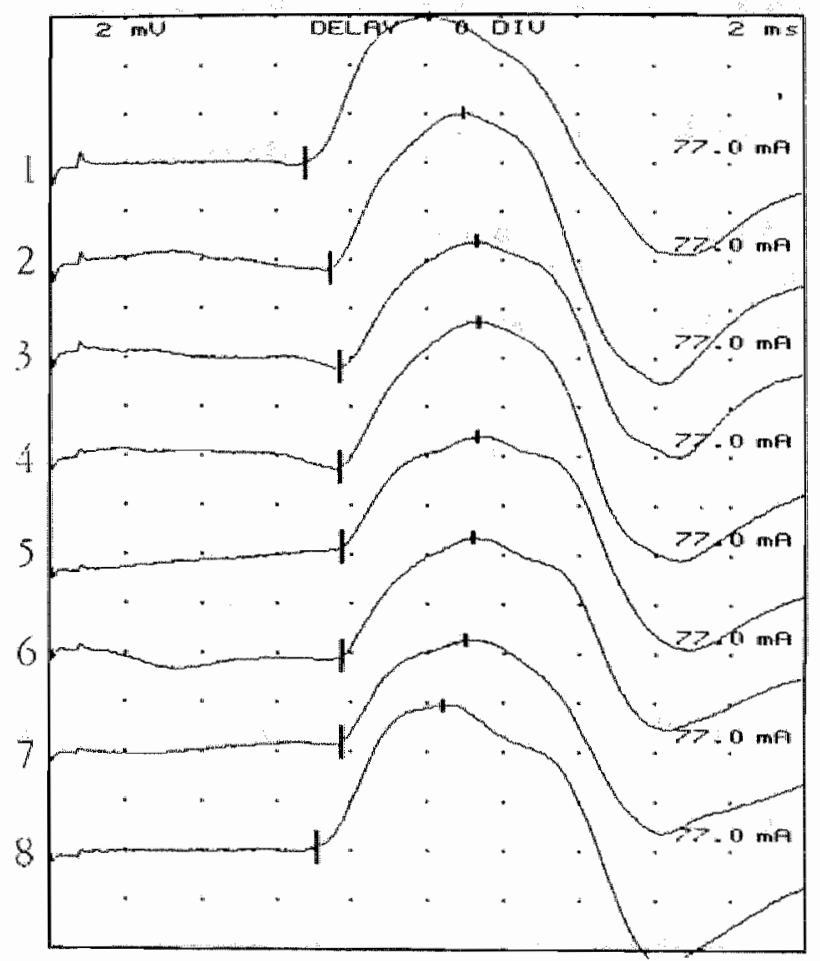

Figure 7-2.

Recording of the compound muscle action potential of the extensor indicis for the only patient who had a major increase in latency during supination. Trace 1 , before supination; trace 8 , after ten seconds of relaxation; and all other traces, during supination, at ten-second intervals. 
though this patient's supination force was relatively low (maximum: $3.8 \mathrm{New}$ ton-meters in the painful arm and 4.4 Newton-meters in the pain-free arm). When this patient was re-examined one month later, an identical increase in latency was found. Fourteen months later, the pain had resolved spontaneously and the latency was just within normal limits ( 0.4 millisecond).

\subsection{Concentric-needle electromyography}

None of the patients had fibrillation potentials in the radially innervated muscles of the forearm. In one patient, an increased percentage of polyphasic motor-unit potentials was found in the extensor digitorum muscle of the painful arm. This was not the same patient who had the increase in latency during resisted supination.

\subsubsection{Operative findings}

During passive pronation, all patients had visible compression of the radial nerve by the superficial part of the supinator muscle. It was impossible to determine whether this compression was abnormal. Alterations in the nerve, such as indentation or swelling, were not noted in any patient. No ruptures of the muscles or of the arcade of Frohse were identified, and all tissues appeared normal. Only one patient (case 10) had good relief of pain from the procedure. In three patients (case 2, 3 and 16) a fair result was recorded.

\subsection{DISCUSSION}

Compression neuropathy of the posterior interosseous nerve has been demonstrated to be due to various causes, including simple ganglion, hemangioma arising from the radial recurrent vessels, lipoma, rheumatoid disease associated with a well defined arcade of Frohse, neoplasm and inflammatory swelling (Llewellyn Bowen and Stone 1966, Mulholland 1966, Sharrard 1966, Spinner 1968, Kopell and Thompson 1976, Spaans 1987). The clinical features of a lesion of this motor branch of the radial nerve include pain on palpation of the site of compression and weakness of the extensor carpi ulnaris, extensor digitorum and more distal muscles innervated by the radial nerve, without sensory loss. Extension of the fingers is weak, but there is no wrist drop, since the nerve supply to the extensor carpi radialis longus is intact. On extension the wrist tends to drift into radial deviation because of weakness of the extensor carpi ulnaris (Spinner and Spencer 1974). 
Refractory tennis elbow without paresis of extensor muscles are also thought to be caused by compression of the posterior interosseous nerve (Capener 1966, Sharrard 1966, Roles and Mausley 1972, Kopell and Thompson 1976, Hagert et al. 1977, Lister et al. 1979). The compression is assumed to be most frequently caused by the fibrous edge of the superficial part of the supinator muscle, the arcade of Frohse (Spinner 1968, Roles and Maudsley 1972, Lister et al. 1979). At that site, Werner et al. (1980) recorded pressures of 40 to 50 millimeters of mercury ( 5.33 to 6.67 kilopascals) exerted on the nerve during passive stretching of the supinator muscle. Contraction of the muscle caused by tetanic nerve stimulation led to pressures that were about 4 times higher. Experimental studies have demonstrated a reduction of venous blood-flow at pressures of 20 to 30 millimeters of mercury ( 2.67 to 4 kilopascals), ischemia at 60 to 80 millimeters of mercury ( 8 to 10.66 kilopascals), and blockade of axonal transport at 50 millimeters of mercury (6.67 kilopascals). Blockade of conduction of nerve impulses occurs at pressures of 130 to 150 millimeters of mercury (17.33 to 20 kilopascals) (Werner et al. 1980). Studies of patients who had radial tunnel syndrome noted that most had jobs that elicited high cyclic strain with rotatory movements of the forearm. This suggests that dynamic compression of the nerve by the supinator muscle during rotation of the forearm may elicit the pain in the radial tunnel syndrome.

Local tenderness along the radial nerve where it passes under the arcade of Frohse is considered to be the distinguishing characteristic between radial tunnel syndrome and typical tennis elbow (Roles and Maudsley 1972, Werner 1979). This criterion was not used in the study by van Rossum et al. (1978) which may have been responsible for the lack of electromyographic findings in their patients. In order to select the most typical cases for our study we used the criteria of Werner, which include this sign.

The result of decompression of the radial nerve was good in only one of the ten patients whom we operated on, and it was fair in three. Roles and Maudsley (1972) reported a good result in 35 of 38 elbows (36 patients). Werner (1979) obtained a good result in 60 of 90 elbows after a follow-up of six months and in 73 of 90 elbows after a follow-up of twenty-four months. Hagert et al. (1977) reported good or excellent relief of pain after 42 of 50 procedures. Lister et al. (1979) obtained a good result after 19 of 20 procedures. Heyse-Moore (1984) considered the decompression to have been successful in 10 of 13 elbows, and Ritts et al. (1980) reported good results after 20 of 39 procedures. These results could be regarded as evidence in support of the entrapment hypothesis. HeyseMoore (1984) as well as Wadsworth (1987), however, emphasized that division of the superficial part of the supinator muscle also relieves tension on the lateral 
epicondyle and its adjacent structures, thus allowing relief of symptoms independently of decompression of the radial or of the posterior interosseous nerve. We share this view; in our opinion improvement after an operation cannot be considered a gold standard for the diagnosis of radial tunnel syndrome.

In the absence of distinct weakness of the extensor muscles, the only way to establish a compression of the nerve is by demonstration of local slowing of nerve conduction or of abnormalities on needle-electromyographic studies, or both. These tests have proved to be sensitive in several well defined nerveentrapment syndromes. In our 16 patients, who had long-standing, clinically typical radial tunnel syndrome, we found a slight abnormality on needleelectromyographic testing in only one patient and local slowing of nerve conduction, only during forced supination, in another, whereas electrophysiological testing was unremarkable in all 14 remaining patients.

Most patients who have this syndrome have pain on resisted supination. This seems to be an inherent limitation when the radial nerve is tested during active contraction of the muscle. It also may limit the patient's cooperation, resulting in a relatively weak compression of the posterior interosseous nerve, with little or no effect on nerve conduction. However, most of our patients exerted normal forces; the only positive test was in a patient who had an increase in latency even though the maximum supination force was relatively low (Table 7-2).

In agreement with our results, Rosén and Werner (1980) found normal conduction of impulses in the posterior interosseous nerve in 28 patients when the arms were at rest. On resisted supination, an increase in latency of more than 0.5 millisecond occurred more frequently in the painful arms than in the pain-free arms. After operative decompression of the nerve, the results of the test showed no improvement, although clinical improvement occurred in most of their patients. Hence, we do not believe that their findings support the entrapment hypothesis convincingly.

As Ritts et al. (1987) stated, there appears to be an interrelated clinicopathologic spectrum of disease between so-called radial tunnel syndrome and tendinitis of the common extensors. A musculotendinous lesion of the common extensor tendon at or near the attachment to the lateral epicondyle is regarded as the most frequent cause of tennis elbow (Chard and Hazleman 1989). Radial tunnel syndrome may involve an analogue of this lesion in the supinator muscle or in the origin of the extensor carpi radialis brevis, which is blended with the superficial part of the supinator (Heyse Moore 1984, Van Mameren and Drukker 1984 and Van Mameren et al. 1985). 
Thus, our study does not support the hypothesis that the signs and symptoms in most patients who have a diagnosis of radial tunnel syndrome are caused by compression of the posterior interosseous nerve.

\subsection{CONCLUSIONS}

From this study it may be concluded that:

- in a group of patients who had a diagnosis of radial tunnel syndrome electrophysiological testing was unremarkable in 14 out of 16 patients.

- a slight needle-EMG abnormality was found in only one of the 16 patients, and a local slowing of nerve conduction, only during forced supination, in another patient.

- the data do not support the hypothesis that the signs and symptoms of the majority of cases diagnosed as radial tunnel syndrome are caused by a compression of the posterior interosseous nerve. 
CHAPTER

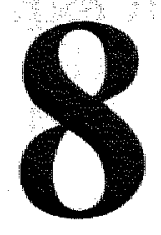

General conclusions 


\section{GENERAL CONCLUSIONS}

\subsection{INCIDENCE AND PREVALENCE}

The studies described in chapters 3 and 4 of this thesis show that tennis elbow is a common condition. The prevalence rate of tennis elbow complaints in the general population between 20 and 80 years of age was $2.0 \%$ and on the basis of this prevalence the incidence was estimated to be between 1 and $2 \%$ per year. The incidence in general practice was half of that of the general population. The incidence of tennis elbow at the orthopaedic out-patient clinic was estimated to be 7 to $9 \%$ of the incidence of the general population. The data about the occurrence of tennis elbow are summarized in figure 8-1.

These findings indicate that approximately 50 per cent of all persons with tennis elbow complaints are treated by the general practitioner, while less than 10 per cent are treated at the orthopaedic outpatient clinic.

In general, these findings emphasize the self-limiting and rather benign character of tennis elbow. There is only a relatively small group of patients with prolonged or severe incapacitating pain at the lateral elbow. The duration of complaints ranges from several weeks to many years. For some patients the economic consequences may be serious. Half of the patients with a job who were seen at the out-patient orthopaedic clinic could not carry out their professional activities at the time of the first consultation. However, this high percentage is caused by selection; patients who cannot perform essential professional activities are more often referred by the general practitioner than other patients. 


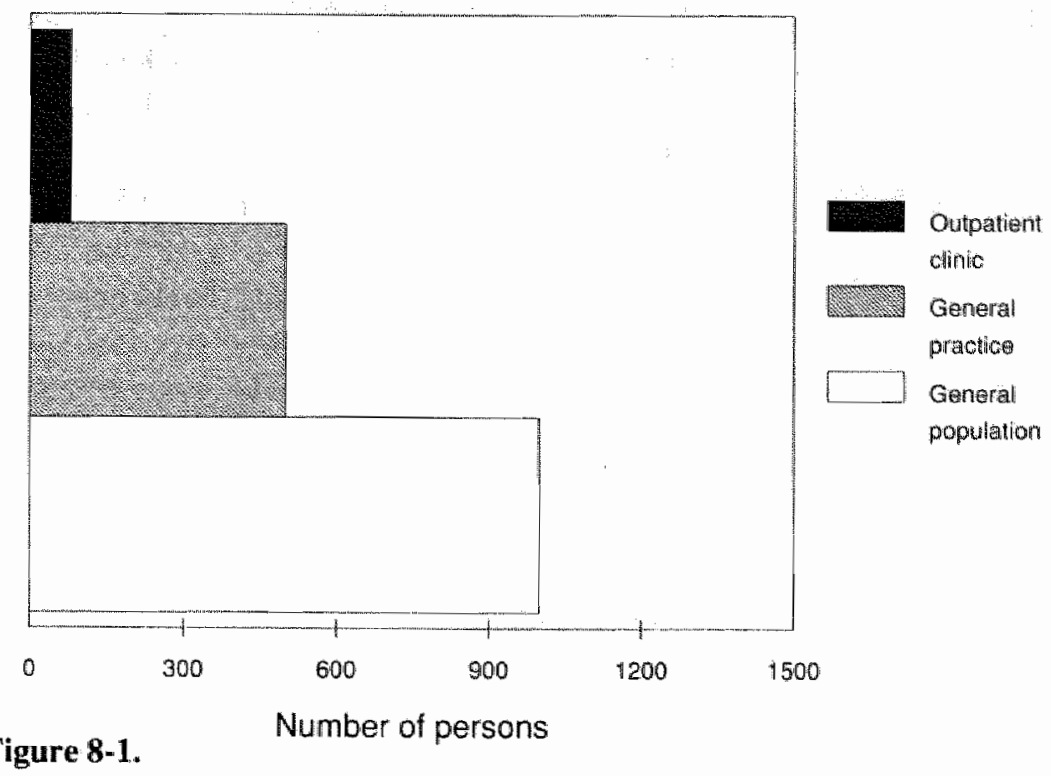

Diagrammatic representation of the prevalence and incidence of tennis elbow in general population, general practice and outpatient department. The number of persons with a tennis elbow are presented for a population of 100,000 persons.

Those patients cannot afford a prolonged period of rest, and medical advice is more often sought to reduce the period of rest.

\subsection{TREATMENT}

In our study (chapter 4), Cyriax' physiotherapy did show some beneficial effects. Similar percentages of favourable results have been reported in the literature about other types of physiotherapy, including placebo physiotherapy. Corticosteroid injections proved to be highly effective in reducing the pain, but this effect was temporary in several patients. However, in view of the limited amount of time required for treatment, the satisfactory level of pain relief and the lack of side effects encountered we tend to prefer the treatment with corticosteroid injections in cases in which rest alone does not provide adequate relief of the tennis elbow complaints. Whatever treatment is applied, restriction of activities should be emphasized.

In our study of the results of operative treatment of tennis elbow (chapter 5), surgical treatment proved to be successful in cases of resistant tennis elbow, leading to a good to excellent result in 77 per cent of patients at the one year 
Table 8-1.

Comparison of the results of Operative versus Conservative treatment of tennis elbow

Outcome parameter

$\begin{array}{lllll} & \text { overall patient"s severity subjective } & \text { local } \\ \text { Predictor } & \text { results } & \text { satisfaction of pain loss of grip tenderness }\end{array}$

Treatment

(Operative versus Conservative) -1.193

0.765

$-0.709$

$-1.118$

$0^{\circ}$ grip strength

Dominance

Severity of pain

Cervical complaints

Subjective loss of grip strength

0.577

$-0.895$

0.518

Tenderness

Resisted wrist dorsiflexion

Resisted extension of digit 3

a) The table gives the significant coefficients from the reduced models; coefficients not entered were not significant.

follow-up and in 90 per cent at the five year follow-up. Our study did not address the question how long patients should be treated conservatively before operation is indicated. The severity of pain, the cause of the complaints, the impairment of work and daily life and the patience of the patient should determine the choice between a more or less aggressive approach.

An ideal comparison between conservative and operative treatment should be based on randomization between these two treatment groups. As with many other disorders, such a comparative study is difficult to perform for tennis elbow. Many patients and physicians will consider operative treatment as a final resort and for that reason will opt for a programme starting with conservative treatment.

In order to obtain some insight into the effectiveness of operative treatment compared to conservative treatment, we used the logistic regression model described in the addendum of chapter 4 (conservative treatment). The results of the logistic regression are presented in table 8-1. The results of treatment at the one-year follow-up were used as outcome parameters. Two treatment groups were formed, one conservatively treated group $(n=74)$ and one group of operated 
patients ( $\mathrm{n}=62)$. Other pre-treatment variables were allso used as predictors. This analysis was done to explain variations in the outcome parameters from variations in the predictors.

In table 8-1 only statistically significant values are included. Thus, table 8-1 shows that the kind of treatment is the most important factor determining outcome parameters. The operated patients had a significantly better result than the non-operated patients on all outcome parameters at the one year follow-up. The other predictor which significantly influenced the results was subjective loss of grip strength. Patients with a high subjective loss of grip strength had less favourable results than other patients.

It is tempting to conclude that operative treatment has a significantly more beneficial effect on tennis elbow complaints than conservative treatment. Many known and unknown variables, however, may have influenced the selection of patients for operative treatment and not all of them have been included in our logistic regression model. We conclude, nevertheless, that the good results of operative treatment we have found in many severe cases not responding to conservative treatment may provide good arguments against the therapeutic nihilism after failure of conservative treatment suggested by some authors (Kamien 1990).

\section{Personal opinion about treatment scheme of tennis elbow}

Based upon the results of the studies in chapters 3,4 , and 5 I have formulated the following personal opinion about the preferred treatment scheme for tennis elbow (Table 8-2).

Our study in the general population showed that only half of the patients seek medical attention. The physicians should use this intrinsic self-healing mechanism and prescribe rest. Symptomatic treatment may be prescribed in the form of NSAIDs. In addition, local ice application may be used in acute cases with severe pain and local swelling. On the basis of our own results with Cyriax' treatment as well as the reports of other authors in the literature we tend to advise a reduction of the use of physiotherapy in the treatment of tennis elbow.

Corticosteroid injections proved to be effective in reducing the pain of tennis elbow. Because of the theoretical side effects it may be advisable to use these injections only in patients with moderate or severe pain. These injections should only be used after failure of a period of rest. 
Tabie $8 \% 2$

Proposed treatment scheme for tennis elbow

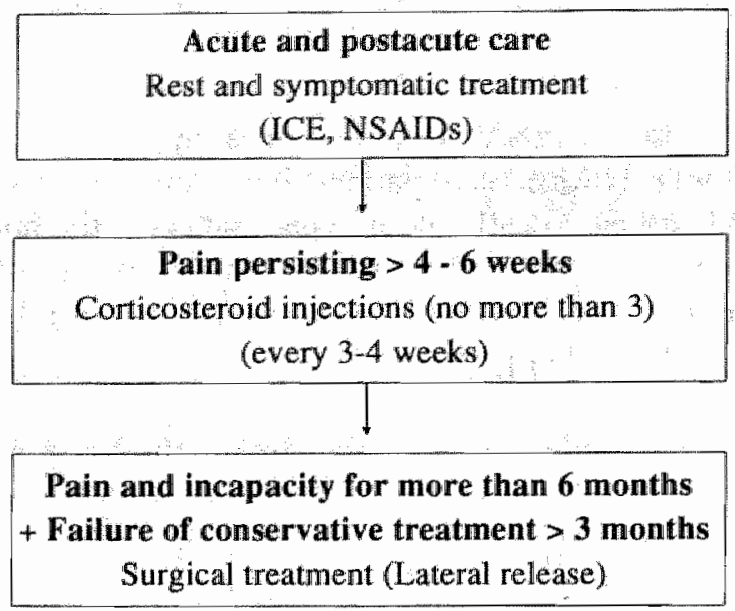

Operative treatment proved to be successful and may be considered after 6 months of pain and incapacity due to tennis elbow. This period is arbitrary. A shorter period will lead to operative treatment of patients who would have recovered without operation. A longer period will lead to an unnecessary period of pain or sick leave. Based upon our own results we make a plea for a rather simple lateral release procedure under local anaesthesia.

In addition to this treatment scheme, we suggest that:

- after 12 weeks of treatment the general practitioner should consider referral (chapter 3).

- the medical specialist should not prescribe physiotherapy for tennis elbow if the general practitioner has already prescribed it.

\subsection{FURTHER RESEARCH}

Many questions have remained unanswered, and many others have been raised by our studies.

Our study of the radial tunnel syndrome (Chapter 7) led us to the question whether the so-called radial tunnel syndrome might be caused by a lesion in the supinator muscle or in the origin of the extensor carpi radialis brevis muscle or the septum between the extensor carpi radialis brevis muscle and extensor 
digitorum muscle. The latter septum blends with the superficial part of the supinator. This hypothesis fits in with the anatomical concept of force transmission in the lateral ellbow complex described in Chapter 2 . These concepts may serve as a general framework for tennis elbow and tennis elbow-like complaints. The study described in Chapter 6, using a physical examination based on these concepts, was unfortunately not conclusive on that point. Histological proof of these theories seems necessary but is difficult to obtain due to previously described technical and ethical problems and to problems in choosing the correct site for biopsies. Because the Lateral Cubital Force Transmission System (LCFTS) can be identified on MRI-scans, it is theoretically possible to localize lesions. Further research might be carried out in this direction. Because limited data are available about the way forces are conducted in the LCFTS in different positions of ellbow flexion, this might also be a subject for further research.

We think that this kind of anatomical and pathophysiological research may further clarify the pathology of these conditions. This might lead to a better selection of patients who are at risk of having a prolonged period of lateral elbow pain. It may also lead to a more causal and selective treatment of the condition. 



\section{CHAPTER}

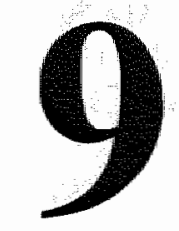

\section{Summary}

This thesis reports the findings and results of five studies on anatomical, epidemiological and clinical aspects of tennis elbow.

Chapter 1 gives an overview of the most important literature data of tennis elbow. Little general agreement exists concerning the nature of the underlying pathological changes. The most commonly supported view is that tennis elbow is caused by macroscopic or microscopic ruptures in the common extensor origin, which induce a repair reaction, sometimes involving the space beneath the conjoined tendon. Intra-articular elbow lesions, compression of the radial nerve and cervical radiculopathy, however, have also been suggested as causes of tennis elbow. Diagnosis and epidemiology are discussed in this chapter. Opinions on the treatment of tennis elbow vary. Many modalities of conservative and operative treatment are in use, but a scientific prove of their effectiveness is often lacking. Literature data are used as the basis for a discussion of the results of conservative and operative treatment.

Chapter 2 outlines the relevant anatomy of the elbow and the detailed anatomy of the lateral region in particular. Recent anatomical studies have led to the concept of the Lateral Cubital Force Transmission System, which describes the way tensile forces generated by the muscle fibres of the forearm muscles are transmitted to the lateral part of the humerus. 
Chapter 3 describes the results of an epidemiological study of the prevalence of tennis elbow complaints in the general population. The study was performed in a group of persons referred to the University Hospital Maastricht for conditions not related to tennis elbow. 708 persons were studied and a prevalence of 4.4 per cent was found. Extrapolation of the data to the general population resulted in a prevalence of tennis elbow complaints of 2.0 per cent. The prevalence for women aged between 40 and 50 was 11.4 per cent. 45 per cent of the persons studied had never been treated for their tennis elbow. Chapter 3 also describes the results of a study performed in 19 general practices with a total patient population of 47,500 . In a one-year period 247 patients were seen for the first time with a tennis elbow. This corresponded to an incidence of 5.2 patients per 1000 patients in the general practice. Restriction of painful activities was the therapy most frequently prescribed by the general practitioner $(68 \%) .28$ per cent of the patients received physiotherapy and 38 per cent had corticosteroid injections. At the one year follow-up, 47 per cent of patients were cured and 18 per cent had improved. Nearly 16 per cent of the patients with a tennis elbow were referred to the out-patient clinic.

Chapter 4 reports on a study of the results of conservative treatment in the orthopaedic out-patient clinic. A clinical trial was performed comparing the outcome and side effects in patients with tennis elbow randomised to treatment with local corticosteroid injections or to the physiotherapy method described by Cyriax. The trial included 106 patients. At the 6 week follow-up the corticosteroid group was much more improved than the physiotherapy group with regard to the following evaluation parameters: severity of pain, occurrence of pain, subjective loss of grip strength, resisted dorsiflexion of the wrist and the middle finger, local tenderness, patient's assessment and overall result rating. If patients had cervical complaints the results of treatment were negatively influenced ( $p<0.05$ ). After successful treatment, 34 per cent of the patients in the corticosteroid group had recurrent symptoms, as opposed to 11 per cent of the patients in the physiotherapy group. However, the recurrence rate in the physiotherapy group was much lower, because the group of patients with good or excellent results was rather small. At the 1 year follow-up, 56 patients were satisfied about the results of treatment $(53 \%) ; 30$ patients were moderately satisfied (28\%) and 20 patients $(19 \%)$ were dissatisfied. On the basis of the evaluation criteria described, 25 per cent of the patients had an excellent result. The result in 39 per cent of the patients was considered to be moderate or poor.

The value of operative treatment is assessed in Chapter 5, which presents the results of a group of 63 patients operated upon for tennis elbow and followed prospectively for five years. At the one year follow-up 1 patient had died, but 
the remaining 62 patients were all seen. At the five year follow-up, another 2 patients had died and 3 patients were not seen for follow-up. Thus, at 5 years, 57 patients were seen for follow-up.

In all but 6 patients the extensor origin was macroscopically normal. One year postoperatively, 41 patients $(66 \%)$ were satisfied about the results of treatment. Eight patients $(13 \%)$ considered the results unsatisfactory. Five years after operation, 52 patients $(91 \%)$ were satisfied about the result of treatment, while 2 patients remained dissatisfied with the outcome of treatment of their tennis elbow. In terms of the evaluation criteria at the one year follow-up, 31 patients $(50 \%)$ had an excellent result, while 17 patients had a good result, 10 patients a moderate result and 4 patients a poor result: Five years postoperatively, 33 $(59 \%)$ patients had an excellent result, $18(32 \%)$ a good result, $5(9 \%)$ a moderate and $1(2 \%)$ a poor result. No preoperative parameters were found to correlate significantly with the results of the surgical treatment.

Chapter 6 describes a study, which was performed in order to investigate whether the suspected pathology in tennis elbow could be localized using a diagnostic system based on the concept of the Lateral Cubital Force Transmission System (LCFTS) of the elbow. 101 patients were examined. Accurate physical examination based on the LCFTS seemed to lead to the selection of a group which had a poorer outcome of treatment, but the differences in the results of treatment between this group and the remaining patients were statistically non-significant.

Chapter 7 reports on an electromyographic and nerve conduction study in 16 patients who fully met the clinical criteria for a radial tunnel syndrome. This syndrome has been suggested to be caused by intermittent compression of the posterior interosseous nerve by the supinator muscle. Conduction measurements of the posterior interosseus nerve were performed using surface electrodes for stimulation and recording. The muscle potential was recorded before, during, and after 1 minute of supination at 50 per cent of the maximum force. To obtain reference values, 36 asymptomatic subjects without a history of lateral ellbow pain were investigated. Only 1 of the 16 patients was found to have a significant delay in conduction, which is compatible with compression of the radial nerve. In the remaining 15 patients with clinically suspected compression of the radial nerve, compression was not confirmed. These findings do not support the hypothesis that the signs and symptoms of the majority of cases diagnosed as radial tunnel syndrome are caused by a compression of the posterior interosseous nerve. 
In Chapter 8 the overall findings of this thesis are briefly discussed and recommendations for treatment are given. The results for patients who underwent an operation are compared with those of patients who were not operated upon in a statistical analysis, using a logistic regression model. The results of operative treatment were significantly better than those of non-operative treatment. These results argue against a nihilistic therapeutic approach if conservative treatment fails. Surgical treatment seems to be fully justified in resistant cases. It may, however, not be concluded from our material that operative treatment is indicated at an earlier point in time than we have been using. 


\section{CHAPTER}

\section{0}

\section{Samenvatting}

In dit proefschrift worden de bevindingen en de resultaten beschreven van vijf onderzoeken naar anatomische, epidemiologische en klinische aspecten van de behandeling van de tenniselleboog (epicondylitis lateralis).

In hoofdstuk 1 wordt een overzicht gegeven van de literatuur over de tenniselleboog. Er bestaat geen eenstemmigheid over de pathologie, die aan de tenniselleboog ten grondslag ligt. De opvatting dat de tenniselleboog veroorzaakt wordt door macroscopische en microscopische rupturen in de origo van de extensoren van de onderarm, wordt het meest gesteund. Deze rupturen zouden een reparatie reactie induceren die zich ook zou kunnen uitbreiden naar de subtendineuze ruimte onder de origo van de extensoren van de onderarm. Intra-articulaire elleboogaandoeningen, een compressie van de nervus radialis en aandoeningen van de cervicale wervelkolom zouden volgens de literatuur ook tenniselleboogklachten kunnen veroorzaken. De diagnose en de epidemiologie van de tenniselleboog worden eveneens in hoofdstuk 1 besproken. De behandeling blijkt controversieel. Vele conservatieve en operatieve behandelingen worden toegepast, maar de meeste van hen missen een goede wetenschappelijke bewijsvoering van de effectiviteit. 
De relevante anatomie van het ellebooggewricht, in het bijzonder die van de laterale zijde worden besproken in hoofdstuk 2. Recent anatomisch onderzoek heeft geleid tot de beschrijving wan het "Laterale Krachtgeleidings Systeem" van de elleboog "De wijze waarop trekkrachten gegenereerd door de spiervezelbundels van de onderarmsspieren worden doorgeleid naar de laterale zijde van de humerus, wordt in dit model beschreven.

De resultaten van een epidemiologisch onderzoek naar de prevalentie van tenniselleboogklachten in de algemene bevolking worden beschreven in hoofdstuk 3. Het onderzoek werd werricht in een groep personen, die naar het Academisch Ziekenhuis Maastricht werd verwezen in verband met een aandoening, die geen relatie had met het voorkomen en optreden van tenniselleboogklachten. 708 personen namen aan dit onderzoek deel. De prevalentie van tenniselleboogklachten was $4,4 \%$. Op grond hiervan werd een prevalentie van $2 \%$ voor de gehele bevolking berekend. In de onderzochte groep was de prevalentie onder vrouwen van 40 tot 50 jaar $11,4 \%$. Ruim $45 \%$ van de mensen met klachten bleek niet voor de tenniselleboogklachten te zijn of te worden behandeld. In ditzelfde hoofdstuk worden ook de resultaten van een onderzoek beschreven, dat in 19 huisartsen praktijken met totaal 47.500 patiënten werd verricht. In een periode van één jaar werden door de deelnemende huisartsen 247 patiënten geregistreerd met een tenniselleboog. Dit komt overeen met een incidentie van 5,2 patiënt per 1000 patiënten in de huisartsen praktijk. De belangrijkste therapeutische maatregel van de huisartsen was het voorschrijven wan een beperking van de pijnlijke activiteiten (68\%). $28 \%$ van de patiënten in de onderzochte groep kreeg fysiotherapie en $38 \%$ kreeg lokale injecties met corticosteroïd preparaten. $\mathrm{Na} 1$ jaar bleek $47 \%$ van de patiënten genezen en $18 \%$ verbeterd. Eén op de zes patiënten met een tenniselleboog werd door de huisarts verwezen naar de polikliniek voor specialistische behandeling.

In hoofdstuk 4 worden de resultaten van de conservatieve behandeling op de polikliniek orthopaedie bestudeerd. Een prospectief gerandomiseerd onderzoek werd verricht waarin de resultaten en de bijwerking van de behandeling met corticosteroïd injecties werd vergeleken met de fysiotherapeutische behandeling volgens Cyriax bij 106 patiënten. $\mathrm{Na} 6$ weken behandeling was de corticosteroïd groep significant sterker verbeterd dan de fysiotherapie groep. Significante verschillen werden waargenomen op vrijwel alle parameters. Patiënten met pijnklachten in de nek bleken in beide therapiegroepen minder goede resultaten van de behandeling te ervaren dan patiënten zonder dergelijke klachten $(\mathrm{p}<0.05)$.

$\mathrm{Na}$ succesvolle behandeling kreeg $34 \%$ van de patiënten in de corticosteroïdgroep en $11 \%$ van de patiënten in de fysiotherapie-groep opnieuw klachten. Dit verschill kan worden verklaard uit het feit, dat in de fysiotherapie groep, de groep 
patiènten met een goed of uitstekend resultaat na 6 weken veel klener was dan in de corticosteroïd groep. Eén jaar na het begin van de behandeling waren 56 patiënten tevreden over het resultaat van de behandeling $(53 \%) .30$ patienten waren matig tevreden ( $28 \%$ ) en 20 patienten waren niet tevreden met het behandelingsresultaat. Op basis van de eindevaluatie-criteria had $25 \%$ van de patiënten een uitstekend resultaat. $39 \%$ van de patiènten had volgens deze criteria een matig tot slecht resultaat.

De resultaten van de operatieve behandeling van patiënten met een temniselleboog worden geẻvalueerd in hoofdstuk 5. De operatieresultaten van 63 patienten werden prospectief gedurende vijf jaar vervolgd. Op 6 patiënten na werden bij de operatie geen rupturen in de extensoren origo waargenomen. Eén jaar na de operatie waren 41 patiënten $(66 \%)$ tevreden met het resultaat van de operatie. 8 patiënten $(13 \%)$ waren ontevreden over het resultaat. Vijf jaar na de operatie waren 52 patiënten $(91 \%)$ tevreden over het operatie resultaat, terwijl 2 patiënten ontevreden bleven over het resultaat. Op basis van de eindevaluatie-criteria hadden 31 patiënten na 1 jaar een uitstekend resultaat, 17 patiènten een goed, 10 een matig en 4 een slecht resultaat. Vijf jaar postoperatief hadden 33 (59\%) patiënten een uitstekend resultaat, $18(32 \%)$ een goed, $5(9 \%)$ een matig en 1 $(2 \%)$ een slecht resultaat. In de groep geopereerde patiënten werd geen preoperatieve parameter gevonden die significant correleerde met het eindresultaat van de operatieve behandeling.

In hoofdstuk 6 wordt een onderzoek beschreven met als doel na te gaan of de veronderstelde pathologie van de tenniselleboog kon worden gelokaliseerd binnen het LCFTS met behulp van een daarop gericht lichamelijk onderzoek. 101 patiënten met een tenniselleboog namen aan dit onderzoek deel. De pijn veroorzaakt door palpatie en door het aanspannen van de spieren werd vergeleken voor en één jaar na de behandeling. Het op het LCFTS gebaseerde gedetailleerde lichamelijke onderzoek leek wel in staat een groep patiënten te selecteren, bij wie de behandeling minder succesvol was, maar de verschillen in behandelingsresultaat tussen beide groepen bleken niet statistisch significant.

Hoofdstuk 7 bevat een beschrijving van een electromyografisch en geleidingssnelheidsonderzoek dat werd verricht bij een groep van 16 patiënten, bij wie op klinische criteria de diagnose radialis tunnel syndroom was gesteld. De oorzaak van dit syndroom zou een intermitterende compressie zijn van een tak van de $n$. radialis, de n.interosseus posterior, door de m. supinator.

Geleidingsonderzoek vond plaats met behulp van oppervlakte-elektroden voor stimulatie en afleiding. De spierpotentiaal werd geregistreerd voor, tijdens en na een gedurende 1 minuut volgehouden isometrische supinatie op 50 procent van de maximale supinatiekracht. Om referentiewaarden te verkrijgen werden 36 
klachtenvrije proefpersonen zonder voorgeschiedenis van pijn rond de laterale epicondyl onderzocht. Slechts I van de 16 patiënten had een significante vertraging van de geleidingssnelheid. Bij de overige 15 patiënten met een klinische verdenking op compressie van de $n$. radialis werd de compressie niet bevestigd. De conclusie van dit onderzoek luidt dat in de meerderheid van de patiënten bij wie de diagnose radialis tunnel syndroom is gesteld, de klachten en symptomen niet worden veroorzaakt door een compressie van de nervus interosseus posterior.

In hoofdstuk 8 worden de belangrijkste eindconclusies van de in dit proefschrift beschreven onderzoeken en de daaruit voortwloeiende aanbevelingen voor de behandeling van de tenniselleboog besproken. De resultaten van geopereerde en niet geopereerde patiënten worden vergeleken met behulp van een logistisch regressie model. De resultaten van de operatieve behandeling bleken significant beter dan die van de niet-operatieve behandeling. Dit betekent, dat na het falen van de conservatieve behandeling zeker plaats is voor operatieve behandeling. Of er ook vroeger geopereerd moet worden kan uit dit onderzoek niet worden geconcludeerd. 


\section{CHAPTER}

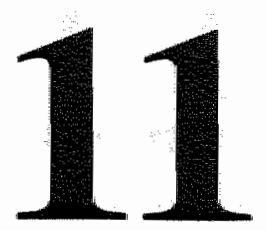

\section{References}

1 Aldes JH. Ultrasonic radiation in treatment of epicondylitis. Gen Pract 1956;13:89-96.

2 Allander E. Prevalence, incidence, and remission rates of some common rheumatic diseases or syndromes. Scan J Rheumatol 1974;3:145 153.

3 Allen JC, Bell H, Shearman CH. Traumatic radiohumeral synovitis. Med J. Aust $1947 ; \mathrm{i}: 48-51$.

4 Bailey RA, Brock BH. Hydrocortisone in temnis elbow-a controlled series. J Roy Soc Med 1957;50:389-390.

5 Baumgard SH, Schwartz DR. Percutaneous release of the epicondylar muscles for humeral epicondylitis. Am J Sports Med 1982;10;233-236.

6 Balogh K. The histologic appearance of corticosteroid injection sites. Arch Pathol Lab Med 1986;110:1168-1172.

7 Behrens F, Shepard N, Mitchell N. Alteration of rabbit articular cartilage by intra-articular injections of glucocorticoids. J Bone Joint Surg 1975,75A:70-76.

8 Beken, van der A, Joveneau B. Traitement chirurgical du tennis-elbow. Acta Orthop Belg 1983;49:161-184.

9 Bengert O. Die Epicondylitis humeri. Z Orthop 1982;120:209-212.

10 Bentley $\mathrm{S}$. The treatment of sports injuries by local injections. Br J Sports Med 1981;15:71-74.

11 Bernhang AM. The many causes of tennis elbow. N Y State J Med 1979;70:1363-1366.

12 Bernhardt M. Uber eine wenig bekannte Form des Beschäftigungsneurialgie. Neurol Zentralb॥ 1896; 15:13.

13 Binder A, Hazleman BL. Lateral humeral epicondylitis- a study of natural history and the effect of conservative therapy. BrJ Rheumatol 1983;22:73-76.

14 Binder A, Parr G, Page Thomas P, Hazleman B. A clinical and thermographic study of lateral epicondylitis. Br J Rheumatol 1983;22:77-81. 
15 Binder A, Hodge G, Greenwood AM, Hazleman BL, Page Thomas DP. Is therapeutic ultrasound effective in treating soft tissue lesions? Br Med J 1985;290:512-514.

16 Binder A, Hazleman BL. Letter to the editor. Br J Rheumatol 1983;22:250.

17 Blanken K. De tenniselleboog. Huisarts en Wetenschap 1981;24:300-303.

18 Bosworth DM. The role of the orbicular ligament in tennis elbow. J Bone Joint Surg $1955 ; 37$ A:527-533.

19 Bosworth DM. Surgical treatment of tennis elbow - a follow-up study. J Bone Joint Surg 1965;47A:1533-1536.

20 Bowden BW. Tennis elbow. J Am Osteopath Assoc 1978;70:45-48.

21 Boyd HB, Mcleod AC. Tennis elbow. J Bone Joint Surg 1973;55A:1183-1187.

22 Brattberg G. Acupuncture therapy for tennis elbow. Pain 1983;16:285-288.

23 Briggs CA, Elliott BG. Lateral epicondylitis - a review of structures associated with tennis elbow. Anat Clin 1985;7:149-153.

24 Bruijn de R. Deep transverse friction; its analgesic effect. Int J Sports Med 1984 suppl; 5:35-36.

25 Cabot A. Tennis elbow, a curable affliction. Orthop Rev 1987;16:322-326.

26 Calvert PT, Allum RL, Macpherson IS, Bentley G. Simple lateral release in treatment of tennis elbow. J Roy Soc Med 1985;78:912-915.

27 Capener $\mathbf{N}$. The vulnerability of the posterior interosseous nerve of the forearm. J Bone Joint Surg 1966;48B:770-773.

28 Carlstedt CA. Mechanical and chemical factors in tendon healing. Acta Orthop Scand 1987:suppl 224:1-75.

29 Carp L. Tennis elbow (epicondylitis) caused by radiohumeral bursitis. Arch Surg 1932;24:905-922.

30 Carroll RE, Jorgensen EC. Evaluation of the Garden procedure for lateral epicondylitis. Clin Orthop 1968;60:201-204.

31 Chard MD, Hazleman BL. Tennis elbow - a reappraisal. Br J Rheumatol 1989;28:186190.

32 Chard MD, Hazleman BL. Pulsed electromagnetic field treatment of chronic lateral humeral epicondylitis. Clin exp rheumatoll 1988;6:330-336.

33 Chard MD, Wright JK, Hazleman BL. Isolation and growth characteristics of adult human tendon fibroblast. Ann Rheum Dis 1987;46:385-390.

34 Clarke RP. Symptomatic, lateral synovial fringe (plica) of the elbow. Arthroscopy 1988:4:112-116.

35 Clarke AK, Woodland J. Comparison of two steroid preparations used to treat tennis elbow, using hypospray. Rheumatol Rehab 1975;14:47-49.

36 Cochran WG. Some methods for strengthening the common $\mathrm{X}^{2}$-tests. Biometrics 1954;10:417-441.

37 Coenen W. Uber ein diagnostisches Zeichen bei der sogenannten Epicondylitis humeri radialis. Z Orthop 1986; 124:323-326.

38 Colt E. Tennis elbow. Br Med J 1970;i:679.

39 Commandre FA, Valdener MV. Tennis-arm: echotomographic confirmation. J Sports Med 1983;23:191-193.

40 Coonrad RW, Hooper WR. Tennis elbow: Its course, natural history, conservative and surgical management. J Bone Joint Surg 1973;55A:1177-1182.

41 Coonrad RW. Tennis elbow. Intr Course Lect 1986;35:94-101.

42 Cowan II, Stone JR. Painful periarticular calcifications at wrist and elbow: diagnosis and treatment. JAMA 1952;149:530-533. 
43 Cyriax JH. The pathology and treatment of temis elbow. $\mathfrak{I}$ Bone and Jomt Surg $1936 ; 18 \mathrm{~B}: 9211-940$.

44 Cyriax J. Treatment by manipulation, massage and injections. In Textbook of or thopaedic medicine. 11 th Ed, London: Ballière Tindall, 1982.

45 Cyriax J, Troisier O. Hydrocortisone and soft-tissue lestions. Br Med J $1953 ; 11: 966-$ 968.

46 Day BH, Govindasamy $\mathbf{N}$, Patnaik $\mathbf{R}$. Corticosteroid injections in the treatment of tennis elbow. Practitioner 1978;220:459-462.

47 De Goes H, Silva O. The radio humeral meniscus and its relation to tennis elbow. Arch Interamerican Rheumatol 1960:3:582-594.

48 Decaix M. Tennis-elbow. Traitement par ablation du ménisque humero-radial. Thèse I"Université de Montpellier 1982.

49 Demark, van RE, Myrabo AK. Calcareous tendinitis at the elbow. Clin Orthop $1956 ; 7: 237-240$.

50 Devereaux MD, Hazleman BL, Page Thomas PP. Chronic lateral humeral epicondylitis - A double-blind controlled assessment of pulsed electromagnetic field therapy. Clin Exp Rheumatol 1985:3:333-336.

51 Dimberg $\mathbf{L}$. The prevalence and causation of tennis elbow (lateral humeral epicondylitis) in a population of workers in an engineering industry. Ergonomics 1987;30:572580.

52 Doran A, Gresham GA, Watson C. Temnis elbow- a clinicopathologic study of 22 cases followed for 2 years. Acta Orthop Scand 1990;61:535-538.

53 EPOZ. Vijfde voortgangsverslag, deel 4, reuma. Instituut Epidemiologie Erasmus Universiteit Rotterdam 1979.

54 Fischer AW. Uber die Epicondylus- und Styloidneuralgie, ihre Pathogenese und zweckmässige Therapie. Arch Klin Chir 1923;125:749-750.

55 Fisherman EW, Feinberg AR, Feinberg SM. Local subcutaneous atrophy. JAMA $1961 ; 179 ; 971-972$.

56 Freeland DE, Gribble M. Hydrocortisone in tennis elbow. Lancet 1954;ii:225-226.

57 Freund E, Gräf H, Schiewe R. Die Epicondylitis humeri. Zentralbl Chir 1970;95:293296.

58 Friedlander HL, Reid RL, Cape RF. Tennis elbow. Clin Orthop 1967;51:109-116.

59 Froimson AI. Treatment of tennis elbow with forearm support band. J Bone Joint Surg 1971:53A:183-184.

60 Froimson AI. Tenosynovitis and tennis elbow. In Green DP. Operative hand surgery, New York 1982, Churchill Livingstone, Inc.

61 Garden RS. Tennis elbow. I Bone Joint Surg 1961;43B:100-106.

62 Gardner RC. Tennis elbow: diagnosis, pathology and treatment. Clin Orthop $1970 ; 72: 248-253$.

63 Gartland JJ. Orthopaedic clinical research. J Bone Joint Surg 1988;70A:1357-1364.

64 Gemert van JGWA. The arthrodesis of the wrist joint. Thesis Catholic University Nijmegen 1984, Nijmegen.

65 Gerberich SG, Priest JID. Treatment for lateral epicondylitis: Variables related to recovery. Br J Sports Med 1985;4:224-227.

66 Gerster JC, Baud CA, Lagier R, Boussina I, Fallet GH. Tendon calcifications in chondrocalcinosis. Arthritis Rheum 1977;20:717-722.

67 Goldie I. Epicondylitis lateralis humeri (epicondylalgia or fennis elbow): a pathological study. Acta Chir Scand 1964;Suppl 339:1-119. 
68 Gondos B. Teninis elbow: a re-valuation. Am I Roentgenol 1958,79:684-691.

69 Gonzette. Essai de pathogenie de l"epicondylite des joueurs de tennis et des escrimeurs. Presse Med 1931:31:1167-1168.

70 Goodfellow $I W$, Bullough $\mathbf{P G}$. The pattern of ageing of the articular cartilage of the ellbow joint. J Bone Joint Surg 1967:44B:175-181

71 Gray RG, Gottlieb NL. Intra-articular corticosteroids- an updated assessment. Clin Orthop $1983 ; 177: 235-263$.

72 Groenewegen PP, Kerssens $\mathbb{J}$, Curfs EC. Fysiotherapie in de Nederlandse gezondheidszorg. Ned T Fysiotherapie 1990;100:9-19.

73 Gruchow HW, Pelletier BS. An epidemiologic study of tennis elbow. Am J Sports Med 1979;7:234-8.

74 Gunn CC, Milbrandt WE. Tennis elbow and the cervicall spine. Can Med Assoc J 1976;114:803-809.

75 Hagert C, Lundborg G, Hansen T. Entrapment of the posterior interosseous nerve. Scand J Plast Reconstr Surg 1977;11:205-212.

76 Halle JS, Franklin RJ, Karalfa BL. Comparison of four treatment approaches for lateral epicondylitis of the elbow. JOSPT 1986;8:62-69.

77 Hamilton AR. Calcification in epicondylar bursa. Br J Surg 1938;26:446-448.

78. Hamilton PG. The prevalence of humeral epicondylitis: a survey in general practice. J R Coll Gen Pract 1986;36:464-465.

79 Hansson KG, Horwich ID. Epicondylitis humeri. JAMA 1930;84:1557-1561.

80 Hermans GPH. Het pathologisch anatomisch substraat wan surmenageletsels, hun lokalisatie en het effect van lokale coticosteroïdinjecties. Geneesk Sport 1977; 10:9-12.

81 Heyse-Moore GH. Resistant tennis elbow. I Hand Surg 1984;9B:64-66.

82 Hohl M. Epicondylitis- Tennis elbow. Clin Orthop 1961;19:232-238.

83 Hohmann. Das Wesen und die Behandlung des sogenannten Tennisellenbogens. Münch Med Wochenschr 1933;80:250-252.

84 Holden $G$, Hume Kendall $P$. The newer corticosteroids for local injection. Ann Physical Med 1961;6:178-182.

85 Hollander $\mathbf{J L}$. Intra-articular hydrocortisone in arthritis and allied conditions. J Bone Joint Surg 1953;35A:983-990.

86 Hughes ESR. Acute deposition of calcium near the elbow. J Bone Joint Surg $1950 ; 32 B: 30-34$.

87 Hughes GRV, Currey HLF. Hypospray treatment of tennis elbow. Ann rheum dis $1969 ; 28: 58-62$.

88 Ifeld FW, Field SM. Treatment of tennis elbow. JAMA 1966;195:111-114.

89 Jalovaara P, Lindholm RV. Decompression of the posterior interosseous nerve for tennis elbow. Arch Orthop Trauma Surg 1989;108:243-245.

90 Janda J, Koudela K. Skelettmuskelbeteiligung bei der Enthesopathie des Epicondylus lateralis humeri (sogenannter Tennis-Ellenbogen). Z Orthop 1988;126:105-107.

91 Jones DM. Tennis elbow. J Bone Joint Surg 1958;40B:825.

92 Jonquière $\mathbf{M}$. De behandeling van schouder- en elleboogsaandoeningen volgens de richtlijnen van Cyriax-een vergelijkend onderzoek in de thuisartsenpraktijk. Thesis Erasmus University 1986, Rotterdam.

93 Kaiser W, Köhnlein HE, Otten G. Behandlung der persistierenden Epicondylitis humeri radialis. Chir Praxis 1982;30;247-253.

94 Kamien M. A rational management of tennis elbow. Sports Med 1990;9:173-191. 
95 Kammer G, Bollinger A. Die operative Behandlung der Epicondylitis-Ruckblick auf 100 operierte Falle. Schweiz Rundschau Med Prax 1981;70.2177-2179.

96. Kaplan EB. Treatment of tennis elbow (epicondylitis) by denervation. J Bone Joint Surg 1959;41A:147-151.

97 Kitai E, Itay S, Rader A, Engel J, Modan M. An epidemiological study of latera! epicondylitis (tennis elbow) in amateur male players. Ann Chir Main 1986;5:1 13-121.

98. Kivi P. The etiology and conservative treatment of humeral epicondylitis. Scan I Rehabil Med 1982;15:37-41.

99 Klimek E. Potato picker's plight. Can Med Assoc J 1984; 130:106.

100 Kopell HP, Stone WAL. Peripherall Entrapment Neuropathies, 2nd edit. New York: RE Krieger Publishing Co, 1976.

101 Koppers B, Riel K. Drei-Phasen-Szintigraphie bei der Epicondylitis lhumeri radialis. Fortschr Röntgenstr 1982;137:147-151.

102 Krahl H, Langhoff J . Degenerative Sehnen-veränderungen nach lokaler Kortikoidanwendung. $Z$ Orthop 1971;109:501-511.

103 Krahl H, Plaue R. Sehnennupturen nach Cortisoninjektionen. Med und Sport $1971 ; 1: 1: 264-267$.

104 Kuipers RKW. Epicondylitis humeri, en de behandeling ervan met lokale histamineinspuitingen. Ned T Geneesk 1943:87:105-109.

105 Kurppa K, Waris P, Rokkanen P. Tennis elbow. Scan J Work Environ \& Health 1979; suppl 3:15-18.

106 Labelle H, Fallaha M, Newman N, Rivard C.-H, Guibert R. The treatment of tennis ellbow-a critical review of the literature. J Bone Joint Surg 1990;72B:536.

107 Lamberts H. De morbiditeitsanalyse 1972 door groepspraktijk Ommoord: een nieuwe ordening van ziekte- en probleemgedrag voor de huisartsgeneeskunde. Huisarts en Wetensohap 1975;18:7-39.

108 Lambrecht W. Wesen und Behandlung der Epicondylitis humeri. Chirurg $1948 ; 19: 55$. 58.

109 Lamphier TA, Pepi J, Covino J, Ostroger J, Rosenthal CE, Brusch C. Prednisolone (meticortelone) in treatment of epicondylitis. Arch Surg 1959;78:492-497.

110 Lataster LMA, Mameren van H, Terwindt E. A plastic embedding procedure for 15 microns sections of large undecalcified tissue blocks. Anat Rec 1992 (in press).

111 Laupacis A, Rorabeck CH, Bourne RB, Sim H, Sim DA. Randomized trials in orthopaedics: Why, How, and When? J Bone Joint Surg 1989;71A:535-543.

112 Leach RE, Miller JK. Lateral and medial epicondylitis of the elbow. Clin Sports Med $1987 ; 6: 259-272$.

113 Lisdonk van der EH, Bosch van den WJHM, Huygen FJA, Lagro-Janssen ALM. Ziekten in de huisartspraktijk. Utrecht, Bunge." 1990.

114 Lister GD, Belsole RB, Kleinert HE. The radial tunnel syndrome. I Hand Surg $1979 ; 4: 52-59$.

115 Litt R, Albassir A. Tennis elbow traitement par allongement des radiaux. Acta Orthop Belg $\llbracket 983 ; 49: 154-160$.

116 Llewellyn Bowen T, Stone KH. Posterior interosseous nerve paralysis caused by a ganglion of the elbow. I Bone Joint Surg 1966;48B:774-776.

117 Lundeberg T, Abrahamsson P, Haker E. A comparative study of continuous ultrasound, placebo ultrasound and rest in epicondylalgia. Scand J Rehabil Med 1988;20:99-101. 
118 Lundeberg $T$, Abrahamsson $\mathbf{P}$, Bondesson L, Haker E. Effect of vibratory stimulation of experinental and clinical pain. Scan J Rehabil Med 1988;20:149-159.

119 Lundeberg $\mathbf{T}$, Haker $\mathbb{E}_{\text {, Thomas }}$ M. Effect of laser versus placebo in tennis elbow. Scan J Rehab Med 1987:19:135-138.

120 Luopajầ ${ }^{1}$, Kuorinka 1, Virolainen M, Holmberg M. Prevalence of tenosynovitis and other work injuries of the upper extremities in repetitive work. Scan JWork Environ Health 1979; suppl 5;48-55.

121 MacNab 1. Rotator cuff tendinitis. Ann Roy Coll Surg Engl 1973;53:271-287.

122 Mameren, van $\mathbf{H}$, Drikker $\mathbf{J}$. A functional anatomical basis of injuries to the ligamentum and other soft tissues around the elbow joint: transmission of tensile and compressive loads. Int J Sports Med Suppl 1984;5:88-92.

123 Mameren, van H, Lataster A, Rensema J, Drukker J. The use of moderne imaging techniques (CT-scanning and NMR) in the study of the locomotor apparatus. Acta Morphol Neeri-Scand 1985;23:247-258.

124 Mankin HJ, Conger KA. The acute effects of intra-articular hydrocortisone on articular cartilage in rabbits. J Bone Joint Surg 1966;48A:1383-1388.

125 Marlin T. Treatment of "tennis elbow" with some observations on joint manipulation. Lancet $1930 ; \mathrm{i}: 509-511$.

126 Mathiowetz V, Weber K, Volland G, Kashman N. Reliability and validity of grip and pinch strength evaluations. J Hand Surg 1984;4A:222-226.

127 McCarty DJ, Hogan JM. Inflammatory reaction after intrasynovial injection of microcrystalline adrenocorticosteroid esters. Arthritis Rheum 1964;7:359-367.

128 Meherin JM, Cooper CE. Tennis elbow. Am J Surg 1950;80:622-625.

129 Meine J, Eicher E. Ergebnisse der Denervationsoperation bei Epicondylitis radialis et ulnaris humeri. Handchir Mikrochir Plast Chir 1981;12:254-259.

130 Michelle AA, Krueger FJ. Lateral epicondylitis of the elbow treated by fasciotomy. Surgery 1956;39:277-284.

131 Miller MH, Topliss DJ. Chronic upper limb pain syndrome (repetitive strain injury) in the australian workforce: a systemic cross sectional rheumatologicall study of 229 patients. J Rheumatol 1988; 15:1705-1712.

132 Mills GP. Treatment of tennis elbow. Br Med J 1928;i:12-13.

133 Mills GP. Treatment of tennis elbow. Br Med J 1937;ii:212-213.

134 Moore M. Radiohumeral synovitis. Arch Surg 1952;64:501-505.

135 Morrey BF, Kai-Nan A. Functionall anatomy of the ligaments of the elbow. Clin Orthop 1985;201:84-90.

136 Morris H. Riders sprain. Lancet $1882 ;$ ii:557.

137 Mulholland RC. Non-tratumatic progressive paralysis of the posterior interosseous nerve. J Bone Joint Surg 1966;48B:781-785.

138 Müller U, Moll G. Uber die Behandlung der Epicondylitis mit lokal injiziertem Orgotein (Doppelblindstudie). Z Rheumatol 1983:42:21-24.

139 Murley AHG. Tennis-elbow-treated with hydrocortisone acetate. Lancet 1954;ii:223225.

140 Murray-Leslie CF, Wright V. Carpal tunnel syndrome, humeral epicondylitis, and the cervical spine: a study of clinical and dimensional relations. Br Med J 1976;i:14391442.

141 Netter FH. The Ciba collection of medical illustrations. Volume 8 - Muskuloskelletal system part 1. Summit, Ciba-Geigy Corporation, 1987. 
142 Nevellos AB. The treatment of tennis elbow with triameinolone acetonide. Current Med Res Opin 1980;6:507-509.

143 Newman JH, Goodfellow JW. Fibrillation of head of radius as one cause of tennis elbow. Br Med J 1975;ii:328-330.

144 Niepall GA, Sit"aj S. Enthesopathy. Clin Rheum Dis 1979;5:857-872.

145 Nirschl RP. Tennis elbow. Orthop Clin N Am 1973;4:787-800.

146 Nirschl RP, Pettrone FA. Tennis elbow -the surgical treatment of lateral epicondylitis. J Bone Joint Surg 1979:61 A:832-839.

147 Nirschl RP. Muscle and tendon trauma: Tennis Elbow. pp 481-96. In: Morrey BF: The elbow and its disorders. Philadelphia: W.B.Saunders Company, 1985.

148 Nollen A. Ergebnisse der operativen Behandlung der Epikondylitis lateralis humeri. Orthopäde 1981;10:328-329.

149 O'Neil J, Sarkar K, Uhthoff HK. A retrospective study of surgical treated cases of tennis elbow. Acta Orthop Belg 1980;46:189-196.

150 Oerke HP, Horst M, Jacobsen U. et al. Gemeinschaftsstudie Epicondylitis humeri. Orthop Praxis 1977;4:265-269.

151 Osgood R. Radiohumeral bursitis, epicondylitis, epicondylagia (tennis elbow). Arch Surg 1922;4:420-433.

152 O'Sullivan S. Tennis-Elbow. Br Med J 1883;II:1168.

153 Paar O, Zweymuller K, Sauer G. Zur Operationstechnik der Epicondylitis humeri radialis. Chirurg 1978;49:520-522.

154 Penners W, Schnitzler M, Kircher E, Göttinger W. Epicondylitis humeri - TennisEllenbogen. Fortschr Med 1977;95:1587-1592.

155 Percey EC, Carson JD. The use of DMSO in tennis elbow and rotatorcuff tendinitis: a double-blind study. Med Sci Sports Exerc 1981; 13:215-219.

156 Peters G. Tools for the measurement of stress and strain field in soft tissue (application to the elbow joint). Thesis, University of Limburg, Maastricht, The Netherlands 1988 .

157 Posch JN, Goldberg VM, Larrey R. Extensor fasciotomy for tennis elbow - A long-term follow-up study. Clin Orthop 1978;135:179-182.

158 Priest JD. Tennis elbow; the syndrome of average players. Minn Med 1976;59:367371.

159 Quin CE, Binks FA. Tennis-elbow (epicondylalgia externa)- Treatment with hydrocortisone. Lancet 1954;ii:221-223.

160 Regan W, Morrey B, Connard R. Microscopic pathology of lateral epicondylitis. J Bone Joint Surg 1990;72B:536.

161 Registratienet Huisartspraktijken. Gezondheids-problemen en Diagnosen in de Huisartspraktijk, rapportage nr.1 1991. Rijksuniversiteit Limburg, Maastricht.

162 Resnik D, Niwayama. Entheses and enthesopathy. Radiology 1983;146:1-9.

163 Richards AJ, Swinson DR. Assessment of tennis-elbow. Lancet 1973;i:1454-1455.

164 Ritts GD, Wood MB, Linschied RL. Radial tunnel syndrome. A ten-year surgical experience. Clin Orthop 1987;219:201-205.

165 Roles NC, Maudsley RH. Radial tunnel Syndrome. Resistant tennis elbow as a nerve entrapment. J Bone Joint Surg 1972;54B:499-508.

166 Rosen I, Werner CO. Neurophysiological investigation of posterior interosseous nerve entrapment causing lateral elbow pain. Electroencephalogr Clin Neurophysiol 1980;50:125-133.

167 Rossum, van J, Buruma OJS, Kamphuisen HAC, and Onvlee GJ. Tennis elbow-at radial tunnel syndrome? J Bone Joint Surg 1978;60B:197-198. 
168 Roto P. Kivi P. Prevalence of epicondylitis and tenosynovitis among meatcutters. Scan I Work Environ Health 1984;:10:203-205.

169 Rumke CL. De nauwkeurigheid van percentages; tabellen met betrouwbaarheidsintervallen. Ned Tijdschr Geneeskd 1976;47:2052-2058.

1:70 Runge F. Zur Genese und Behandlung des Schreiberskrampfes. Berl Klin Wochenschr $1873 ; 10: 245-248$.

$\$ 71$ Saariok T, Eriksson E. Randomized trial of oral naproxen or local injection of betamethason in lateral epicondylitis of the humerus. Orthopedics 1986;9:191-194.

172 Saillant G, Edouard B, Benazet JP, Bombelles F, Roy-Camille R, Neves I. Traitement chirurgical des épicondylalgies rebelles a propos de 126 cas. Ann Chir $1989: 43: 486-490$.

173. Sarkar K, Uhthoff HK. Ultrastructure of the commonextensor tendon in tennis elbow. Virchows Arch A Path Anat Histol 1980;386:317-330.

174 Savastano AA, Kamionek S, Knowles K, Gibson RI. Treatment of resistant tennis elbow by a combined surgical procedure. Int Surg 1972;57:470-474.

175 Schirmer KP, Marcinowski P. Beitrag zur operativen Therapie der Epicondylitis humeri. Zentralbl Chir 1982; 107:853-857.

176 Schmidt RT, Toews JV. Grip strength as measured by the Jamar dynamometer. Arch Phys Med Rehab 1970;51:321-327.

177 Schmitt J. Bursitis calcarea am Epicondylus Externus Humeri. Arch Orthop Trauma Surg 1921;19:215-221.

178 Schonk JWN. Verzekeringsgeneeskundige aspecten bij epicondylalgie. Tijdschr Verzekeringsgeneesk 1985:23:167-171.

179 Sharrard WJW. Posterior interosseous neuritis. J Bone Joint Surg 1966;48B:777-780.

180 Sheehan $\mathbf{N}$, Sheldon F, Marks D. Grip strength and torquometry in the assessment of handfunction in patients with rheumatoid arthritis. Br J Rheumatol 1983;22:158-164.

181 Shilo R, Engel J, Farin I, Horochowski H. Thermography as a diagnostic aid in tennis elbow. Handchir Mikrochir Plast Chir 1969;8:101-103.

182 Sinclair A. Tennis elbow in industry. Br J Ind Med 1965;22:144-148.

183 Smythe H. The "repetitive strain injury syndrome" is referred pain from the neck. J Rheurnatol 1988;15:1604-1608.

184 Snijders CJ, Volkers ACW, Mechelse K, Vleeming A. Provocation of epicondylalgia lateralis (tennis elbow') by power grip or pinching. Med Sci Sports Exerc 1987;19:518523.

185 Snyder-Mackler L, Epler M. Effect of standard and aircast tennis elbow bands on integrated electromyography of forearm extensor musculature proximal to bands. Am J Sports Med 1989;17:278-281.

186 Sommerville EW. Pain in the upper limb. J Bone Joint Surg 1963;45B:621.

187 Spaans F. Compression and entrapment neuropathies. In Handbook of Clinical Neurol-

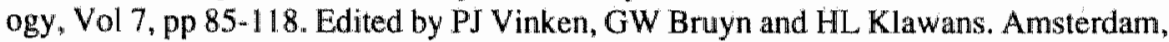
Elsevier Science Publishers, 1987.

188 Spencer GE, Herndon CH. Surgical treatment of epicondylitis. J Bone Joint Surg 1953;35A:421-424.

189 Spinner M. The arcade of Frohse and its relationship to posterior interosseous nerve paralysis. J Bone Joint Surg 1968;50B:809-812.

190 Spinner M, Spencer P. Nerve compression of the upper extremity; A clinical and experimental review. Clin Orthop 1974;104;46-67. 
191 Stack JK. Acute and chronic bursitis in the region of the elbow. Surg Clin N Am $1949: 29: 155-162$.

192 Stovell PB, Beinfield MS. Treatment of resistant lateral epicondylitis of the elbow by lengthening of the extensor carpi radialis brevis tendon. Surg Gynecol Obstet $1979 ; 149+526-528$.

193 Strizak AM, Gleim GW, Sapega A, Nicholas JA. Hand and forearm strength and its relation to tennis. Am J Sports Med 1983;11:234-239.

194 Thörmer HJ, Weber K. Die Hohmannsche Operation, eine zuverlässige Behandlungsmethode der Epicondylicis lateralis humeri. Zentralbl Chir 1957:26:10761079 .

195 Thorngren KG, Werner CO. Normal grip strength. Acta Orthop Sciand 1979;50:255. 259.

196 Thurtle OA, Tyler AK, Cawley MID. Grip strength as a measure of response to treatment for lateral epicondylitis. Br J Rheumatol 1984;23:154.

197 Tönnis D. Die Entstehung der Epicondylitis humeri (Elektromyographische Befunde). Orthop Praxis 1977:4:253-254.

198 Tönnis D. Elektromyographische Untersuchungen zur Frage berufsabhängiger Erkrankungen von Machinenschreibkrafften. Arch Orthop Trauma Surg 1965;58:56-81 .

199 Trethowan WN. Anyone for tennis elbow. Occupat Health Saf 1986;38:74-75.

200 Trethowan WH. Tennis elbow. Br med J 1929:1218.

201 Uhthoff HK, Sarkar K, Maynard JA. Calcifying tendinitis. Clin Orthop 1976;118:164-168.

202 Uhthoff HK, Sarkar K. A re-appraisal of tennis elbow. Acta Orthop Belg 1980;46:7482.

203 Valk, van der FA. Sociale uitkeringen veroorzaakt door ziektes van het steun-en bewegingsapparaat. Voordracht 25 jarig bestaan Instituut voor Orthopaedie Nijmegen 1987.

204 Valtonen EJ. Methylprednisolone acetate in the management of periarthritis of the shoulder and external humeral epicondylitis resistant to physical therapy. Ann Med Int Fenn 1967;56:61-63.

205 Verhaar JAN, Walenkamp G.H.I.M. Operative treatment of tennis ellbow. Acta Orthop Scand 1985;56;451.

206 Verhaar JAN, Van der Linden AJ. De tenniselleboog. Reuma Wereldwijd 1986;10:46.

207 Verhaar J, Van Mameren H, Brandsma A. The risks of neurovascular injury in elbow arthroscopy - Starting anteromedially on anterolaterally? Arthroscopy $1991 ; 7(3): 287$ 290.

208 Wadsworth TG. Tennis elbow" conservative, surgical, and manipulative treatment. Br" Med J 1987;294:621-624.

209 Wadsworth TG. The elbow. Edinburgh, Churchill Livingstone, 1982.

210 Wal, van der JC. The organization of the substrate of proprioception in the elbow region of the rat. Thesis, University of Limburg, Maastricht, The Netherlands, 1988.

211 Waldis MF. Der Eingriff nach G.Hohmann an Ellbogen-operative Behandlung eines Symptoms? Z Orthop 1989;127:606-610.

212 Wanivenhaus A. Differentialdiagnose der Epicondylitis humeni radialis. $Z$ Orthop 1986:124:775-779. 
213 Wamivenhaus A. Kickinger W, Zweymiller K. Die Epicondylitis humeri radialis unter besondere Berücksichtigung der Operation nach Wilhelm. Wiener Klin Wochenschr $1988,98: 338-341$.

214 Waris $\mathbf{P}$, Kuorinka I, Kurppa K, Luopajarwi T, Virolainen M, Pesonen K, Nummil J, Kukkonen R. Epidemiologic screening of occupationall neck and upper limb disorders. Scan I Work Environ Health 1979;5 (suppl 3):25-38.

215 Weh L, Baumann A. Polymyographische Ableitungen bei der Epicondylopathia humeri radialis. Z Orthop $1989,127.72-76$.

216 Werner CO. Lateral elbow pain and posterior interosseous nerve entrapment. Acta Orthop Scand 1979;suppl 174:1-62.

217 Werner $\mathrm{CO}$, Haeffner $\mathbf{F}$, Rosen 1. Direct recording of local pressure during passive stretch and active contraction of the supinator muscle. Arch Orthop Trauma Surg $1980 ; 96: 299-301$.

218 Wessels D. Die Epikondylitis humeri radialis. Akt Neurol 1981;8:141-144.

219 West EF. Termis elbow. Med J Aust 1938;i:330-331.

220 Wiesner H. Die Epicondylitis lateralis humeri und ihre Behandlung unter besondere Berícksichtigung der Hohmannschen Operation. Zentralbl Chir 1952; 19:787-791.

221 Wilhelm A. Das Radialisirritationssyndrom: Handchir Mikrochir Plast Chir $1970 ; 2: 139-142$.

222 Wilhelm A, Gieseler A. Die Behandlung der Epicondylitis humeri radialis durch Denervation. Chirurg 1962;33:118-122.

223 Williams PL, Warwick R. Gray's Anatomy, ed 37, Edinburgh, Churchill Livingstone, 1989.

224 Winckworth CE. Lawn-tennis elbow. Br Med J 1883; II:708.

225 Wolf de AN, van Meurs A.J. Kleine kwalen: de tenniselleboog. Ned Tijdschr Geneesk $1984 ; 128: 2191-2194$.

226 Wood PHN, Sturrock AW, Badley EM. Soft tissue rheumatism in the community. Clin Rheum Dis 1979;5:743-753.

227 Yerger B, Turner T. Percutaneous extensor tenotomy for chronic tennis elbow: An office procedure. Orthopedics 1985;8:1261-1263.

228 Young HH, Ward L, Henderson ED. Use of hydrocortisone acetate. J Bone Joint Surg 1954;36A:606-607. 
Dit promotieonderzoek vond plaats op de afdeling orthopaedie van het Academisch Ziekenhuis Maastricht. De opzet van de studie kwam tot stand onder begeleiding van prof.dr. A.J. van der Linden, dr. G.H.I.M. Walenkamp, dr. $H$. van Mameren. Graag will ik iedereen dank zeggen voor de steun en de hulp bij mijn promotieonderzoek. Ik waardeer deze belangeloze inzet van zo velen zeer.

Professor Ton van der Linden, promotor, een bijzonder woord van dank aan jou. Als mijn opleider, associé en nu als promotor heeft jouw inzet en zelf-discipline mij tot voorbeeld gediend. Jouw relativerend vermogen is in staat om conflicten te neutraliseren en daardoor tot de kern van de zaak te komen.

Dr. Henk van Mameren, co-promotor en anatoom-arts, mijn welgemeende dank. Heel veel uren heb je in het onderzoek en in het doorlezen van mijn manuscripten gestoken. Als 'echte' wetenschapper heb je het vermogen elke versie weer te lezen alsof het de eerste was. Mede daardoor werd het steeds beter. Daarnaast was de oorspronkelijkheid van je ideeën een plezierige bron van discussie.

Dr. Geert Walenkamp, co-promotor en collega, ook aan jou een persoonlijk dankwoord. Vanaf het eerste uur ben je bij dit onderzoek betrokken geweest. Voor een groot deel van het huisartsenonderzoek heb je je persoonlijk ingezet. Je opbouwend oordeel heeft me gesteund.

Dr. Amold Kester, statisticus, ook jij verdient mijn dank. Dit onderzoek kreeg je uit de erfenis van een collega-statisticus en het omvatte niet alleen statistische analyse, maar ook het 'gewone handwerk' van de 'routine-uitdraaien'. Je bent me behulpzaam geweest op alle gebieden van data-management en data-verwerking. 
Prof. Frank Spaans, klinisch neurofysioloog, mijn dank voor ons gezamenlijk onderzoek naar het Radialis Tunnel Syndroom. Zoals je werk vereist, ben je zeer nauwkeurig en perfectionistisch en ik vond dit erg stimulerend. Aan ons gezamenlijk onderzoek bewaar ik dan ook goede herinneringen.

Dr. Carel Thijs, epidemioloog, dank voor de bereidheid jouw en mijn onderzoek te laten samenvallen en de adviezen voor de uitwerking van de data en het kritisch doorlezen van het hoofdstuk epidemiologie.

Coen Rijks, Appie Wijnen, Mirjam Lamerichs-Geelen en Hugo Corstjens, fysiotherapeuten in het AZM, mijn oprechte dank voor jullie medewerking. De laatste tijd is het niet ongewoon om effecten van fysiotherapie te meten en te publiceren. Jullie waren echter al bereid aan zo'n onderzoek mee te werken, toen thier elders nog nauwelijks sprake van was.

De leden van de promotiecommissie, Prof. Drukker, Prof. Kootstra, Dr. Nollen, Prof. v. Ree en Prof. Rozing, dank voor de beoordeling van het manuscript en de waardevolle suggesties voor correcties.

Verder will ik nog dank zeggen aan:

De nog niet genoemde collega-stafleden Ruud Geesink, André van Ooy, Henk Arens en Sjoerd Bulstra voor de gelegenheid geboden tot de uitwerking van dit proefschrift.

Alle huisartsen die deelgenomen hebben aan het huisartsenonderzoek.

Hans Rensema, illustrator op de afdeling anatomie van de RL, voor het vervaardigen van de fraaie anatomische afbeeldingen die in dit proefschrift verwerkt zijn. Harrie Curvers, die als student-assistent betrokken was bij de data-invoer van de trial en een deel van de uitwerking van het onderzoek in de huisartsenpraktijk.

De medewerkers van de polikliniek orthopaedie, van het dagcentrum en van de AV-dienst van het AZM.

TENSLOTTE, will ik in mijn dank zeker ook mijn ouders en gezin betrekken.

Mijn ouders zijn in alle fase van mijn leven en opleiding zeer geïnteresseerd geweest in het wel en wee en dus ook in dit proefschrift. U ziet, het is gelukt en een groot deel van de basis is door U gelegd.

Mijn echtgenote Joke en mijn kinderen, Esther en Alexander, verdienen mijn grootste dank. Ze hadden niet alleen veel geduld met mij, maar ze waren en blijven ook mijn grootste stimulans. De regelmatig terugkerende vraag van Esther en Alexander "Is je boekje nou nog niet klaar ?!?" liet vaak een niet doofbare echo na.

Ja, nu wel ... 
Jan Verhaar werd geboren op 23 november 1954 in Alkemade. In 1973 behaalde hij het einddiploma gymnasium $\beta$ aan het Bonaventura College te Leiden. In hetzelfde jaar begon hij zijn studie geneeskunde aan de Medische Faculteit van de Rijksuniversiteit te Leiden. In 1980 behaalde hij het artsdiploma.

$\mathrm{Na}$ de vooropleiding algemene heelkunde op de chirurgische afdeling van het Medisch Centrum Alkmaar (opleider: dr. P. de Ruiter) vond de orthopaedische opleiding plaats op de afdeling orthopaedie van het Academisch Ziekenhuis Maastricht (hoofd: prof.dr. A.J. van der Linden).

Registratie als orthopaedisch chirurg volgde in 1987. Sindsdien is hij werkzaam als staflid op de afdeling orthopaedie van het AZM met als bijzondere aandachts-gebieden de reumachirurgie en de behandeling van aandoeningen van het kniegewricht. 